\title{
Asymmetric synthesis and chiroptical properties of enantiopure helical ferrocenes
}

\author{
Antonio Urbano,* Ana M. del Hoyo, Alicia Martínez-Carrión, M. Carmen Carreño \\ antonio.urbano@uam.es
}

\section{Experimental Procedures}

General. Melting points were obtained in open capillary tubes and are uncorrected. ${ }^{1} \mathrm{H}$ - and ${ }^{13} \mathrm{C}-\mathrm{NMR}$ spectra were recorded in $\mathrm{CDCl}_{3}$ at 300 and $75 \mathrm{MHz}$, respectively. All reactions were monitored by thin layer chromatography that was performed on precoated sheets of silica gel 60, and flash column chromatography was done with silica gel 60 (230-400 mesh) of Merck. Eluting solvents are indicated in the text. The apparatus for inert atmosphere experiments was dried by flaming in a stream of dry nitrogen. Ethyl ether, $\mathrm{CH}_{2} \mathrm{Cl}_{2}$ and THF were dried over $4 \AA$ molecular sieves. All other reagent quality solvents were used without purification. For routine workup, extraction was carried out with ethyl acetate, and solvent drying with $\mathrm{MgSO}_{4}$.

Measurements. UV/vis spectra were recorded in on a JASCO V-660 spectrophotometer and circular dichroism (CD) spectra were measured with JASCO J-815 CD spectropolarimeter. The spectra were measured in $\mathrm{CH}_{2} \mathrm{Cl}_{2}$ in a quartz cuvette $(1 \mathrm{~cm})$ at 298 o $\mathrm{K}$.

\section{General Procedure A. Suzuki coupling.}

To a mixture of enantiopure 2-( $p$-tolylsulfinyl)ferrocenyl boronic acid, $\left(S_{\mathrm{S}}, S_{\mathrm{p}}\right)-7^{1}(227 \mathrm{mg}, 0.62 \mathrm{mmol})$, $\mathrm{PdCl}_{2}$ (dppf) (45 mg, $0.06 \mathrm{mmol}$ ) and $\mathrm{NaOH}(100 \mathrm{mg}, 2.5 \mathrm{mmol})$, a solution of the corresponding iodo/bromo aromatic derivative 8 a-f $(1.25 \mathrm{mmol})$ in toluene $(7.7 \mathrm{~mL})$ and water $(3.8 \mathrm{~mL})$ was added. The

(1) (a) Jensen, J. F.; Sotofte, I.; Sorensen, H. O.; Johannsen, M. J. Org. Chem. 2003, 68, 1258. (b) Seitzberg, J. G.; Dissing, C.; Sotofte, I.; Norrby, P.-O.; Johannsen, M. J. Org. Chem. 2005, 70, 8332. 
resulting suspension was refluxed overnight and, after workup and flash chromatography, the pure coupled products $9 a-f$ were obtained.

\section{General Procedure B. Formylation.}

To a solution of the corresponding sulfoxide $(0.94 \mathrm{mmol})$ in THF $(9.8 \mathrm{~mL})$ at $-78{ }^{\circ} \mathrm{C}$, tert-BuLi $(0.82 \mathrm{~mL}, 1.4$ mmol, $1.7 \mathrm{M}$ in pentane) was added dropwise during $10 \mathrm{~min}$. The resulting solution was stirred for 10 min at the same temperature before addition of DMF $(0.82 \mathrm{~mL}, 10.4 \mathrm{mmol})$. After stirring at $-78{ }^{\circ} \mathrm{C}$ for 2 $h$, the solution was quenched with water. After workup and flash chromatography, the pure aldehydes were obtained.

\section{General procedure C. Corey-Fuchs homologation.}

A solution of $\mathrm{PPh}_{3}(390 \mathrm{mg}, 1.5 \mathrm{mmol})$ and $\mathrm{CBr}_{4}(250 \mathrm{mg}, 0.75 \mathrm{mmol})$ in $\mathrm{CH}_{2} \mathrm{Cl}_{2}(15 \mathrm{~mL})$ was stirred for 10 min at $0{ }^{\circ} \mathrm{C}$. Then, a solution of the corresponding aldehyde $(0.60 \mathrm{mmol})$ in $\mathrm{CH}_{2} \mathrm{Cl}_{2}(15 \mathrm{~mL})$ was added, and the reaction mixture was stirred for $2 \mathrm{~h}$ at this temperature. After filtration over silica gel and solvent elimination, $\mathrm{Et}_{2} \mathrm{O}(20 \mathrm{~mL})$ and $\mathrm{THF}(10 \mathrm{~mL})$ were added under nitrogen. The solution was cooled at $-78{ }^{\circ} \mathrm{C}$ and $n$-BuLi $2.5 \mathrm{M}(0.86 \mathrm{~mL}, 2.09 \mathrm{mmol})$ was added dropwise. After $30 \mathrm{~min}$, the mixture was allowed to warm to room temperature, stirred for $1 \mathrm{~h}$ and hydrolyzed with water. After workup and solvent elimination, the crude was purified by flash chromatography affording the corresponding ethynyl compounds.

\section{Ohira-Bestmann reagent (11). ${ }^{2}$}

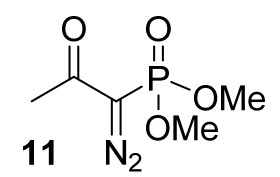

A solution of $\mathrm{TsCl}(8 \mathrm{~g}, 42.0 \mathrm{mmol})$ and $\mathrm{NaN}_{3}(2.73 \mathrm{~g}, 42.0 \mathrm{mmol})$ in acetone $(120 \mathrm{~mL})$ and $\mathrm{H}_{2} \mathrm{O}(120 \mathrm{~mL})$ was stirred at $0{ }^{\circ} \mathrm{C}$ for $2 \mathrm{~h}$. Acetone was evaporated, the reaction mixture was extracted with diethyl ether, and the organic phase was dried with $\mathrm{Na}_{2} \mathrm{SO}_{4}$. Evaporation of solvent gave tosyl azide (97\%) as a colourless oil.

(2) (a) Ohira, S. Synth. Commun. 1986, 19, 561. (b) Müller, S.; Liepold, B.; Roth, G. J.; Bestmann, H. J. Synlett 1996, 521. 
A solution of $\mathrm{NaH}$ ( $60 \%$ dispersion in mineral oil, $0.99 \mathrm{~g}, 41.5 \mathrm{mmol}$ ) in THF $\left(100 \mathrm{~mL}\right.$ ) was cooled at $0{ }^{\circ} \mathrm{C}$. Dimethyl (2-oxopropyl)phosphonate $(6.26 \mathrm{~g}, 37.7 \mathrm{mmol})$ in THF $(100 \mathrm{~mL})$ was added dropwise, and the solution was stirred at $0{ }^{\circ} \mathrm{C}$ for $1 \mathrm{~h}$. Tosyl azide $(8.18 \mathrm{~g}, 41.5 \mathrm{mmol})$ was added in one portion and the resulting mixture was stirred at $0{ }^{\circ} \mathrm{C}$ for $30 \mathrm{~min}$. The reaction mixture was quickly passed through a short column of silica gel (EtOAc) to give Ohira-Bestmann reagent in 96\% yield ( $36.2 \mathrm{mmol}, 6.95 \mathrm{~g}$ ).

${ }^{1} \mathrm{H}$ NMR $\delta\left(300 \mathrm{MHz}, \mathrm{CDCl}_{3}\right): 3.75(\mathrm{~s}, 3 \mathrm{H}), 3.71(\mathrm{~s}, 3 \mathrm{H}), 2.15(\mathrm{~s}, 3 \mathrm{H})$.

\section{General Procedure D. Seyferth-Gilbert homologation.}

The corresponding aldehyde $(0.36 \mathrm{mmol})$, dimethyl (1-diazo-2-oxopropyl)phosphonate (Ohira-Bestmann reagent 11) (354 mg, $1.84 \mathrm{mmol}), \mathrm{K}_{2} \mathrm{CO}_{3}(101.8 \mathrm{mg}, 0.74 \mathrm{mmol})$ were suspended in dry $\mathrm{MeOH}(2.82 \mathrm{~mL})$ and stirred for $48 \mathrm{~h}$, at rt. After evaporation of the solvent, the crude was partitioned between $\mathrm{CH}_{2} \mathrm{Cl}_{2}$ and brine. The combined organic layers were dried $\left(\mathrm{Na}_{2} \mathrm{SO}_{4}\right)$, evaporated, and the residue was purified by flash chromatography affording the pure alkyne.

\section{General procedure E. Cycloisomerization}

A mixture of the corresponding alkyne $(0.15 \mathrm{mmol})$ and $\mathrm{PtCl}_{2}(4 \mathrm{mg}, 0.015 \mathrm{mmol})$ in dry toluene $(2.2 \mathrm{~mL})$, under argon, was heated at $100{ }^{\circ} \mathrm{C}$ for the time indicated in each case. The crude mixture was filtered through celite and washed with hexane. After solvent removal and flash chromatography using a short pad of neutralized silica gel eluting with hexane, the ortho-condensed aromatic ferrocenes were obtained, as red solids.

$\left(S_{\mathrm{S}}, S_{\mathrm{p}}\right)$-2-Phenyl-1-( $p$-tolylsulfinyl)ferrocene (9a).

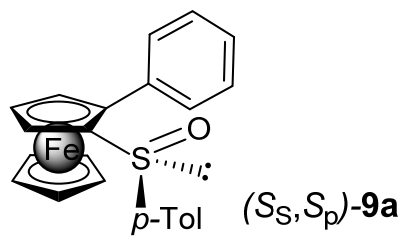


Compound $\left(S_{p}, S_{S}\right)$-9a was obtained from commercially available iodo-benzene (8a) following general procedure $\boldsymbol{A}$ (eluent hexane/EtOAc/ $\left.\mathrm{CH}_{2} \mathrm{Cl}_{2} 79: 22: 8\right)$, in $79 \%$ yield $(0.49 \mathrm{mmol}, 210 \mathrm{mg}$ ), as an orange solid. The physical and spectroscopic data agreed with those published in the literature. ${ }^{3}$

${ }^{1} \mathrm{H}$ NMR $\left(300 \mathrm{MHz}, \mathrm{CDCl}_{3}\right): \delta 7.74(\mathrm{~d}, J=8.1 \mathrm{~Hz}, 2 \mathrm{H}), 7.64(\mathrm{~d}, J=8.1 \mathrm{~Hz}, 2 \mathrm{H}), 7.32-7.19(\mathrm{~m}, 5 \mathrm{H}), 4.66$ (broad s, 1H), 4.34 (broad s, 1H), 4.07 (s, 5H), 4.05 (broad s, 1H), 2.36 (s, 3H).

$\left(S_{\mathrm{p}}\right)$-1-Formyl-2-phenylferrocene (10a).

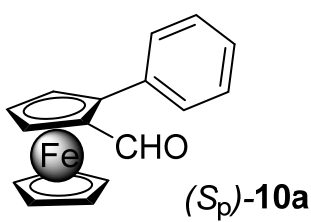

Compound $\left(S_{p}\right)$-10a was obtained from sulfoxide $\left(S_{p}, S_{S}\right)$-9a following general procedure $\boldsymbol{B}$ (eluent hexane/EtOAc 5:1), in 64\% yield ( $0.6 \mathrm{mmol}, 174 \mathrm{mg})$, as an orange oil.

$[\alpha]_{D}^{20}=-697\left(c=0.070, \mathrm{CH}_{2} \mathrm{Cl}_{2}\right)$.

${ }^{1} \mathrm{H}$ NMR (300 MHz, $\left.\mathrm{CDCl}_{3}\right): \delta 10.21(\mathrm{~s}, 1 \mathrm{H}), 7.53(\mathrm{~d}, J=7.8, \mathrm{~Hz}, 2 \mathrm{H}), 7.41-7.29(\mathrm{~m}, 3 \mathrm{H}), 5.00$ (broad s, 1H), 4.84 (broad s, 1H), 4.71 (broad s, 1H), $4.26(\mathrm{~s}, 5 \mathrm{H})$.

${ }^{13} \mathrm{C}$ NMR (75 MHz, CDCl 3 ): $\delta$ 193.1, 136.0, 129.7 (2C), 128.3 (2C), 127.3, 92.7, 75.07, 72.0, 71.1 (5C), 70.0, 68.5 .

MS (FAB+): $m / z(\%) 290\left(\mathbf{M}^{+}, 43\right), 57(30)$.

HRMS: Calculated for $\mathrm{C}_{17} \mathrm{H}_{14} \mathrm{FeO}\left(\mathrm{M}^{+}\right) 290.0394$, found 290.0397 .

$\left(R_{P}\right)$-2-Ethynyl-1-phenylferrocene (6a).

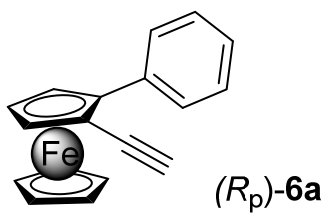

(3) Pedersen, H. L.; Johannsen, M. J. Org. Chem. 2002, 67, 7982. 
Compound $\left(R_{\mathrm{p}}\right)$-6a was obtained from aldehyde $\left(S_{\mathrm{p}}\right)$-10a following general procedure $C$ (eluent hexane), in $60 \%$ yield $(0.36 \mathrm{mmol}, 103 \mathrm{mg})$, as an orange foam.

$[\alpha]_{D}^{20}=-434\left(c=0.08, \mathrm{CH}_{2} \mathrm{Cl}_{2}\right)$.

${ }^{1} \mathrm{H}$ NMR $\left(300 \mathrm{MHz}, \mathrm{CDCl}_{3}\right): \delta 7.81(\mathrm{~d}, J=7.3 \mathrm{~Hz}, 2 \mathrm{H}), 7.33(\mathrm{~m}, 3 \mathrm{H}), 4.62$ (broad s, 2H), 4.33 (broad s, $1 \mathrm{H}$ ), $4.16(s, 5 H), 2.90(s, 1 H)$.

${ }^{13} \mathrm{C} \mathrm{NMR} \mathrm{(75} \mathrm{MHz,} \mathrm{CDCl}_{3}$ ): $\delta$ 137.6, 128.0 (2C), 127.8 (2C), 126.6, 88.0, 82.6, 76.3, 73.2, 71.6 (5C), 68.6, $68.4,63.0$.

MS (EI): $m / z(\%) 286\left(\mathrm{M}^{+}, 100\right), 121(15)$.

HRMS: Calculated for $\mathrm{C}_{18} \mathrm{H}_{14} \mathrm{Fe}\left(\mathrm{M}^{+}\right)$286.0445, found 286.0435 .

$\left(R_{\mathrm{p}}\right)$-Ferrocene[3]helicene (1a).

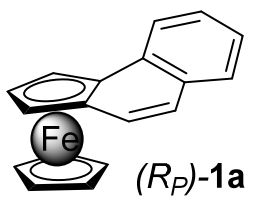

Compound $\left(R_{\mathrm{p}}\right)$-1a was obtained from alkyne $\left(R_{\mathrm{p}}\right)$-6a following general procedure $E(2 \mathrm{~h})$, in $92 \%$ yield (0.14 mmol, $40 \mathrm{mg})$.

$[\alpha]_{D}^{20}=+1037\left(c=0.013, \mathrm{CH}_{2} \mathrm{Cl}_{2}\right),>98 \%$ ee.

${ }^{1} \mathrm{H}$ NMR $\left(500 \mathrm{MHz}, \mathrm{CDCl}_{3}\right): \delta 8.00(\mathrm{~d}, J=7.7 \mathrm{~Hz}, 1 \mathrm{H}), 7.64(\mathrm{~d}, J=7.6 \mathrm{~Hz}, 1 \mathrm{H}), 7.48(\mathrm{t}, J=7.0 \mathrm{~Hz}, 1 \mathrm{H}), 7.41$ $(\mathrm{m}, 2 \mathrm{H}), 7.18(\mathrm{~d}, J=9.1 \mathrm{~Hz}, 1 \mathrm{H}), 5.27$ (broad s, 1H), 4.82 (broad s, 1H), 4.23 (broad s, 1H), $3.74(\mathrm{~s}, 5 \mathrm{H})$.

${ }^{13} \mathrm{C}$ NMR (126 MHz, $\left.\mathrm{CDCl}_{3}\right): \delta$ 135.1, 131.9, 128.6, 127.5, 126.6, 125.2 (2C), 123.3, 83.9, 83.0, 69.3 (5C), $69.2,64.0,61.1$.

MS (EI): $m / z(\%) 286\left(M^{+}, 90\right), 165(100)$.

HRMS: Calculated for $\mathrm{C}_{18} \mathrm{H}_{24} \mathrm{Fe}\left(\mathrm{M}^{+}\right)$286.0445, found 286.0457 .

HPLC: Daicel Chiralpak IB, hexano / 2-propanol 97:3; $0.3 \mathrm{~mL} \mathrm{~min}^{-1}, 254 \mathrm{~nm}, R_{t}=21.2 \mathrm{~min}, T=25^{\circ} \mathrm{C}$ ). 

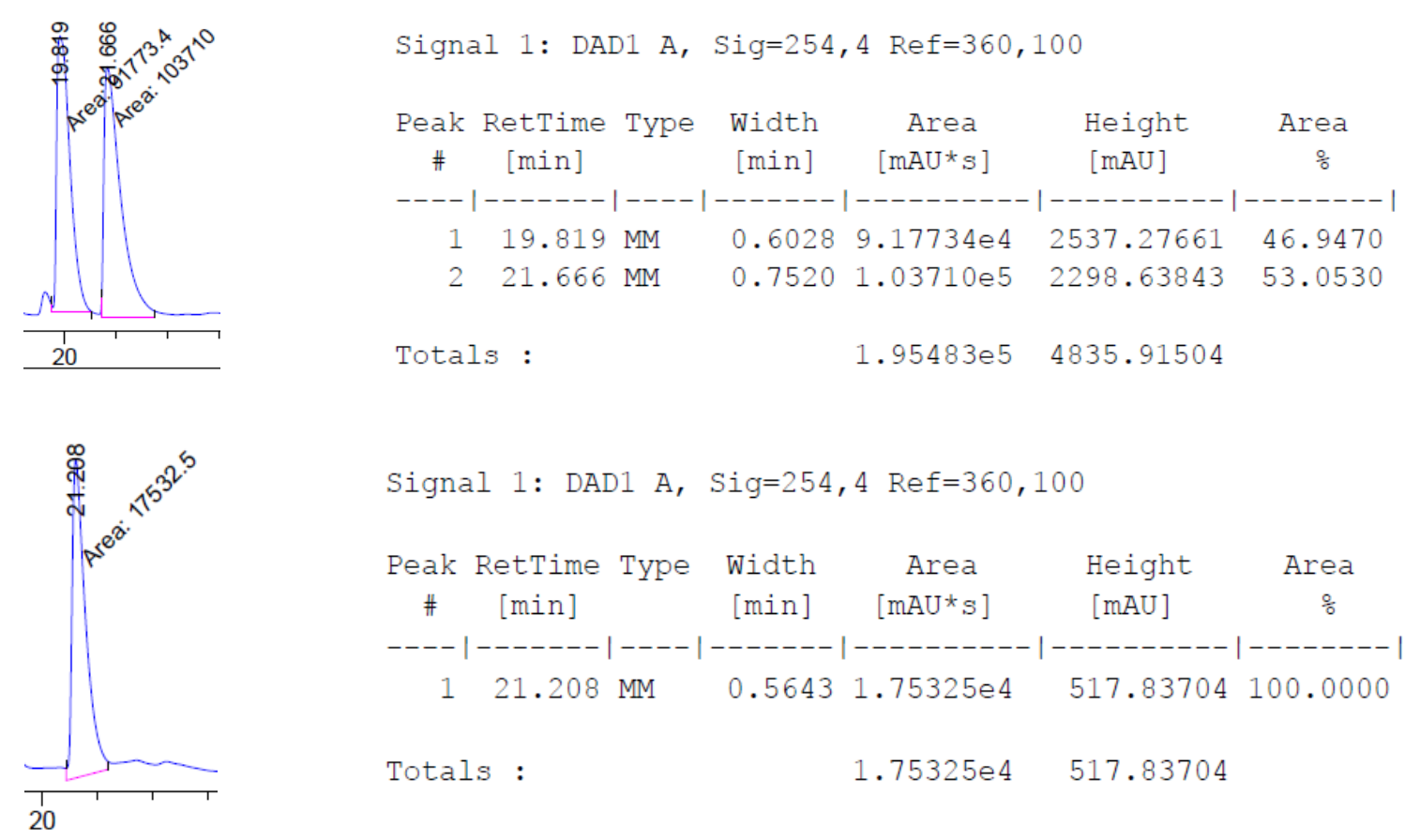

$\left(S_{S}, S_{p}\right)$-2-(4-Fluorophenyl)-1-( $p$-tolylsulfinyl)ferrocene (9b)

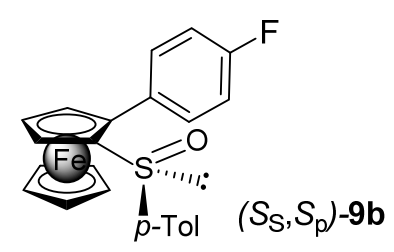

Compound $\left(S_{S}, S_{\mathrm{p}}\right)$-9b was obtained from commercially available 4-iodo-fluorobenzene (8b) following general procedure $\boldsymbol{A}$ (eluent hexane/EtOAc 2:1), in 91\% yield (0,56 mmol, $236 \mathrm{mg}$ ), as an orange solid.

$[\alpha]_{D}^{20}=+39\left(c=0.0075, \mathrm{CH}_{2} \mathrm{Cl}_{2}\right)$.

${ }^{1} \mathrm{H} \mathrm{NMR}\left(300 \mathrm{MHz}, \mathrm{CDCl}_{3}\right): \delta 7.67(\mathrm{dd}, J=8.2,5.6 \mathrm{~Hz}, 2 \mathrm{H}), 7.55(\mathrm{~d}, J=7.9 \mathrm{~Hz}, 2 \mathrm{H}), 7.19(\mathrm{~d}, J=8.3 \mathrm{~Hz}, 2 \mathrm{H})$, $6.93(t, J=8.5 \mathrm{~Hz}, 2 \mathrm{H}), 4.65$ (broad s, $1 \mathrm{H}$ ), 4.36 (broad s, $1 \mathrm{H}), 4.26$ (broad s, 1H), $4.08(\mathrm{~s}, 5 \mathrm{H}), 2.32(\mathrm{~s}, 3 \mathrm{H})$.

${ }^{13} \mathrm{C}$ NMR (75 MHz, CDCl $): \delta 163.5(\mathrm{~d}, \mathrm{~J}=245.0 \mathrm{~Hz}), 141.2,139.6,131.6,131.2(2 \mathrm{C}, \mathrm{d}, \mathrm{J}=8.3 \mathrm{~Hz}), 129.1$, $125.3,115.0(2 \mathrm{C}, \mathrm{d}, J=21.6 \mathrm{~Hz}), 92.1,88.6,72.0,71.0(5 \mathrm{C}), 69.7,69.2,21.3$.

${ }^{19} \mathrm{~F}$ NMR $\left(282 \mathrm{MHz}, \mathrm{CDCl}_{3}\right): \delta-115.15$ 
MS (El+): $m / z(\%) 418\left(M^{+}, 100\right)$

HMRS: Calculated for $\mathrm{C}_{23} \mathrm{H}_{19} \mathrm{FeOSF}\left(\mathrm{M}^{+}\right)$418.0490, found 418.0492 .

$\left(S_{\mathrm{p}}\right)$-2-(4-Fluorophenyl)-1-formyl-ferrocene (10b)

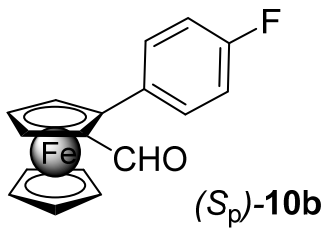

Compound $\left(S_{p}\right)$-10b was obtained from sulfoxide $\left(S_{p}, S_{S}\right)$-9b following general procedure $\boldsymbol{B}$ (eluent hexane/EtOAc 5:1), in 79\% yield (0.74 mmol, $229 \mathrm{mg})$, as an orange oil.

$[\alpha]_{D}^{20}=-200\left(c=0.09, \mathrm{CH}_{2} \mathrm{Cl}_{2}\right)$.

${ }^{1} \mathrm{H}$ NMR $\left(300 \mathrm{MHz}, \mathrm{CDCl}_{3}\right): \delta 10.16(\mathrm{~s}, 1 \mathrm{H}), 7.51(\mathrm{dd}, J=8.6,5.8 \mathrm{~Hz}, 2 \mathrm{H}), 7.05(\mathrm{t}, J=8.7 \mathrm{~Hz}, 2 \mathrm{H}), 4.98(\mathrm{~d}, J$ $=1.0 \mathrm{~Hz}, 1 \mathrm{H}), 4.80($ broad s, $1 \mathrm{H}), 4.70(\mathrm{t}, J=2.5 \mathrm{~Hz}, 1 \mathrm{H}), 4.24(\mathrm{~s}, 5 \mathrm{H})$.

${ }^{13} \mathrm{C} \mathrm{NMR}\left(75 \mathrm{MHz}, \mathrm{CDCl}_{3}\right): \delta$ 192.78, 163.6 (d, $\left.J=258.0 \mathrm{~Hz}\right), 131.9,131.2(2 \mathrm{C}, \mathrm{d}, J=8.4 \mathrm{~Hz}), 115.3(2 \mathrm{C}, \mathrm{d}, J$ $=22.0 \mathrm{~Hz}), 91.4,76.4,73.1,72.0,71.1(5 \mathrm{C}), 68.9$.

${ }^{19}$ F NMR (282 MHz, $\left.\mathrm{CDCl}_{3}\right): \delta-114.68$.

MS (EI+): m/z (\%) 280 (85), $308\left(\mathrm{M}^{+}, 100\right)$

HMRS: Calculated for $\mathrm{C}_{17} \mathrm{H}_{13} \mathrm{FeOF}\left(\mathrm{M}^{+}\right)$308.0340, found 308.0293.

$\left(R_{p}\right)$-2-Ethynyl-1-(4-fluorophenyl)-ferrocene (6b)<smiles>C#CC(=O)Oc1cccc(-c2ccc(F)cc2)c1</smiles> 
Compound $\left(R_{\mathrm{p}}\right)$-6b was obtained from aldehyde $\left(S_{\mathrm{p}}\right)$-10b following general procedure $\boldsymbol{D}$ (eluent heptane/EtOAc 5:1) in 85\% yield (0.31 mmol, $93 \mathrm{mg})$, as an orange solid.

$[\alpha]_{D}^{20}=+13\left(c=0.033, \mathrm{CH}_{2} \mathrm{Cl}_{2}\right)$.

${ }^{1} \mathrm{H}$ NMR $\left(300 \mathrm{MHz}, \mathrm{CDCl}_{3}\right): \delta 7.78(\mathrm{dd}, J=8.7,5.5 \mathrm{~Hz}, 2 \mathrm{H}), 7.03(\mathrm{t}, J=8.7 \mathrm{~Hz}, 2 \mathrm{H}), 4.62$ (broad s, $\left.1 \mathrm{H}\right), 4.54$ (broad s, 1H), $4.32(\mathrm{t}, J=2.5 \mathrm{~Hz}, 1 \mathrm{H}), 4.13(\mathrm{~s}, 5 \mathrm{H}), 2.90(\mathrm{~s}, 1 \mathrm{H})$.

${ }^{13} \mathrm{C}$ NMR (126 MHz, CDCl $): \delta 161.7(\mathrm{~d}, J=249.5 \mathrm{~Hz}), 133.5,129.3(2 \mathrm{C}, \mathrm{d}, J=13.8 \mathrm{~Hz}), 114.9(2 \mathrm{C}, \mathrm{d}, J=$ $21.3 \mathrm{~Hz}), 87.4,82.4,76.3,73.1,71.6(5 \mathrm{C}), 68.4,68.4,62.7$.

${ }^{19} \mathrm{~F}$ NMR (282 MHz, $\left.\mathrm{CDCl}_{3}\right): \delta-115.87$.

MS (EI+): m/z (\%) $304\left(M^{+}, 100\right), 305$ (21)

HMRS: Calculated for $\mathrm{C}_{18} \mathrm{H}_{13} \mathrm{FFe}\left(\mathrm{M}^{+}\right)$304.0351, found 304.0363.

\section{$\left(\boldsymbol{R}_{\mathrm{p}}\right)$-7-Fluoro-ferrocene-[3]-helicene (1b)}

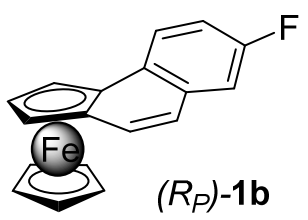

Compound $\left(R_{\mathrm{p}}\right)-\mathbf{1 b}$ was obtained from alkyne $\left(R_{\mathrm{p}}\right)-\mathbf{6 b}$ following general procedure $E(1 \mathrm{~h})$, in $85 \%$ yield (0.13 mmol, $39 \mathrm{mg})$.

$[\alpha]_{\mathrm{D}}{ }^{20}=+510\left(c 0.032, \mathrm{CH}_{2} \mathrm{Cl}_{2}\right) ; e e>98 \%$.

${ }^{1} \mathrm{H}$ NMR $\left(500 \mathrm{MHz}, \mathrm{CDCl}_{3}\right): \delta 7.95(\mathrm{dd}, J=8.4,5.7 \mathrm{~Hz}, 1 \mathrm{H}), 7.44(\mathrm{~d}, J=9.1 \mathrm{~Hz}, 1 \mathrm{H}), 7.30(\mathrm{dd}, J=9.7,2.1$ $\mathrm{Hz}, 1 \mathrm{H}), 7.20(\mathrm{td}, J=8.6,2.3 \mathrm{~Hz}, 1 \mathrm{H}), 7.10(\mathrm{~d}, J=9.1 \mathrm{~Hz}, 1 \mathrm{H}), 5.24$ (broad s, $1 \mathrm{H}$ ), 4.81 (broad s, $1 \mathrm{H}), 4.23$ (broad s, 1H), $3.74(\mathrm{~s}, 5 \mathrm{H})$.

${ }^{13} \mathrm{C}$ NMR (126 MHz, CDCl $)$ : $\delta 160.8(\mathrm{~d}, J=243.0 \mathrm{~Hz}), 133.4(\mathrm{~d}, J=8.3 \mathrm{~Hz}), 131.1,129.3,124.9(\mathrm{~d}, J=8.4$ $\mathrm{Hz}), 124.3,114.5(\mathrm{~d}, J=22.9 \mathrm{~Hz}), 113.7(\mathrm{~d}, J=21.0 \mathrm{~Hz}), 83.2,83.0,69.3(5 \mathrm{C}), 69.3,64.1,61.0$.

${ }^{19}$ F NMR (282 MHz, $\left.\mathrm{CDCl}_{3}\right): \delta-117.21$. 
MS (EI): m/z (\%) $304\left(\mathrm{M}^{+}, 100\right), 183$ (54).

HRMS: Calculated for $\mathrm{C}_{18} \mathrm{H}_{13} \mathrm{FFe}\left(\mathrm{M}^{+}\right)$304.0351, found 304.0343.

HPLC: Daicel Chiralpak IB, $n$-hexane/2-propanol: 90:10; $1.0 \mathrm{~mL} \mathrm{~min}^{-1}, 254 \mathrm{~nm}, R_{t}=29.3 \mathrm{~min}, \mathrm{~T}=25^{\circ} \mathrm{C},>98 \%$ ee.
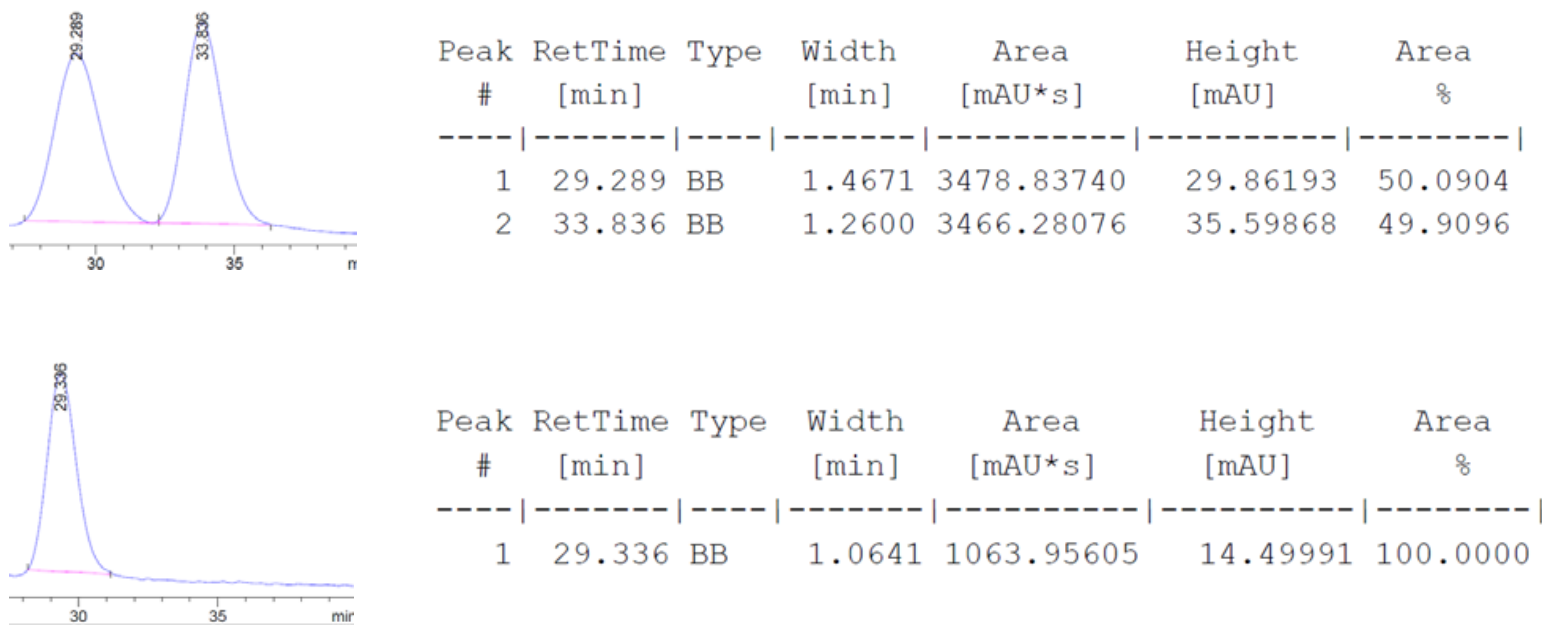

$\left(S_{S}, S_{p}\right)$-2-(4-Methoxyphenyl)-1-(p-tolylsulfinyl)ferrocene $(9 c)$

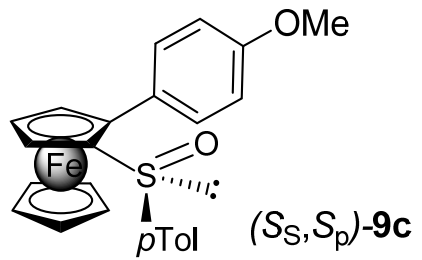

Compound $\left(S_{s}, S_{p}\right)-9 c$ was obtained from commercially available 1-iodo-4-methoxybenzene $(8 \mathrm{c})$ following general procedure $\boldsymbol{A}$ (eluent hexane/EtOAc 5:1), in 51\% yield $(0.32 \mathrm{mmol}, 136 \mathrm{mg})$, as an orange solid. The physical and spectroscopic data agreed with those published in the literature. ${ }^{4}$

${ }^{1} \mathrm{H}$ NMR $\left(300 \mathrm{MHz}, \mathrm{CDCl}_{3}\right): \delta 7.74(\mathrm{~d}, J=8.0 \mathrm{~Hz}, 2 \mathrm{H}), 7.70(\mathrm{~d}, J=7.4 \mathrm{~Hz}, 2 \mathrm{H}), 7.31(\mathrm{~d}, J=8.0 \mathrm{~Hz}, 2 \mathrm{H}), 6.91$ $(\mathrm{d}, J=7.4 \mathrm{~Hz}, 2 \mathrm{H}), 4.65$ (broad s, 1H), 4.37 (broad s, 1H), $4.13(\mathrm{~s}, 5 \mathrm{H}), 4.07$ (broad s, 1H), $3.83(\mathrm{~s}, 3 \mathrm{H}), 2.43$ $(\mathrm{s}, 3 \mathrm{H})$.

(4) Pedersen, H. L.; Johannsen, M. J. Org. Chem. 2002, 67, 7982. 
$\left(S_{p}\right)$-1-Formyl-2-(4-methoxyphenyl)-ferrocene (10c)

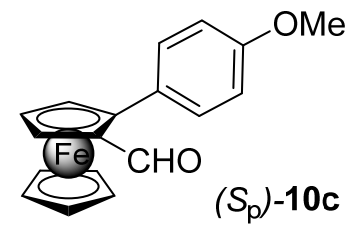

Compound $\left(S_{p}\right)$-10c was obtained from $\left(S_{s}, S_{p}\right)$-2-(4-methoxyphenyl)-1-(p-tolylsulfinyl)ferrocene (9c) following general procedure B (eluent heptane/EtOAc 5:1), in $81 \%$ yield $(0.76 \mathrm{mmol}, 244 \mathrm{mg}$ ), as an orange oil.

$[\alpha]_{D}^{20}=-420\left(c=0.016, \mathrm{CH}_{2} \mathrm{Cl}_{2}\right)$.

${ }^{1} \mathrm{H} \mathrm{NMR} \mathrm{(300} \mathrm{MHz,} \mathrm{CDCl} 3$ ): $\delta 10.19(\mathrm{~s}, 1 \mathrm{H}), 7.45(\mathrm{~d}, J=8.5 \mathrm{~Hz}, 2 \mathrm{H}), 6.90(\mathrm{~d}, J=8.5 \mathrm{~Hz}, 2 \mathrm{H}$ ), 4.96 (broad s, 1H), 4.78 (broad s, 1H), 4.68 (broad s, 1H), $4.23(\mathrm{~s}, 5 \mathrm{H}), 3.84(\mathrm{~s}, 3 \mathrm{H})$.

$\left.{ }^{13} \mathrm{C} \mathrm{NMR} \mathrm{(75} \mathrm{MHz,} \mathrm{CDCl}_{3}\right): \delta$ 193.1, 158.9, 130.7 (2C), 127.8, 113.7 (2C), 92.6, 76.5, 74.4, 71.7, 70.9 (5C), $68.1,55.2$.

MS (EI+): m/z (\%) 249 (18), 277 (29), $320\left(\mathrm{M}^{+}, 100\right)$.

HMRS: Calculated for $\mathrm{C}_{18} \mathrm{H}_{16} \mathrm{FeO}_{2}\left(\mathrm{M}^{+}\right)$308.0340, found 308.0293.

\section{$\left(R_{\mathrm{p}}\right)$-2-Ethynyl-1-(4-methoxyphenyl)-ferrocene (6c)}

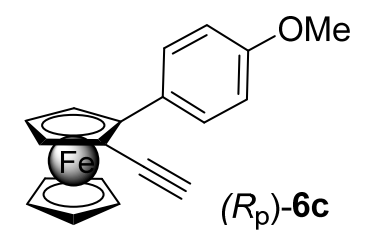

Compound $\left(R_{\mathrm{p}}\right)$-6c was obtained from $\left(S_{\mathrm{p}}\right)$-1-formyl-2-(4-methoxyphenyl)-ferrocene (10c) following general procedure $\boldsymbol{D}$ (eluent heptane/EtOAc 5:1) in $61 \%$ yield $(0.22 \mathrm{mmol}, 70 \mathrm{mg}$ ), as an orange oil.

$[\alpha]_{D}^{20}=+38\left(c=0.014, \mathrm{CH}_{2} \mathrm{Cl}_{2}\right)$.

${ }^{1} \mathrm{H}$ NMR (300 MHz, $\left.\mathrm{CDCl}_{3}\right): \delta 7.76(\mathrm{~d}, J=8.7 \mathrm{~Hz}, 2 \mathrm{H}), 6.90(\mathrm{~d}, J=8.7 \mathrm{~Hz}, 2 \mathrm{H}), 4.60$ (broad s, $\left.1 \mathrm{H}\right), 4.52$ (broad s, 1H), 4.30 (broad s, 1H), $4.14(\mathrm{~s}, 5 \mathrm{H}), 3.85(\mathrm{~s}, 3 \mathrm{H}), 2.91(\mathrm{~s}, 1 \mathrm{H})$. 
${ }^{13} \mathrm{C}$ NMR (75 MHz, CDCl $)$ ): $\delta$ 158.4, 129.6, 128.9 (2C), 113.5 (2C), 88.4, 82.8, 76.0, 72.8, 71.4 (5C), 68.2, $68.1,62.4,55.2$.

MS (EI+): m/z (\%) 301 (17), $316\left(M^{+}, 100\right), 317$ (21).

HMRS: Calculated for $\mathrm{C}_{19} \mathrm{H}_{16} \mathrm{FeO}\left(\mathrm{M}^{+}\right)$316.0551, found 316.0559.

\section{$\left(R_{p}\right)$-7-Methoxy-ferrocene-[3]-helicene (1c)}

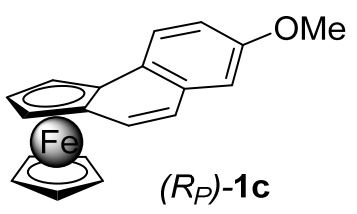

Compound $\left(\boldsymbol{R}_{p}\right)$-1c was obtained from 2-ethynyl-4-methoxyphenyl-ferrocene $(\mathbf{6 c})$ following general procedure $E(1 \mathrm{~h})$, in $82 \%$ yield $(0.12 \mathrm{mmol}, 39 \mathrm{mg})$, as a red solid.

$[\alpha]_{D}^{20}=+1880\left(c=0.016, \mathrm{CH}_{2} \mathrm{Cl}_{2}\right), e e>98 \%$.

${ }^{1} \mathrm{H}$ NMR $\left(500 \mathrm{MHz}, \mathrm{CDCl}_{3}\right): \delta 7.91(\mathrm{~d}, J=8.4 \mathrm{~Hz}, 1 \mathrm{H}), 7.38(\mathrm{~d}, J=9.0 \mathrm{~Hz}, 1 \mathrm{H}), 7.11(\mathrm{~m}, 3 \mathrm{H}), 5.20$ (broad s, 1H), 4.77 (broad s, 1H), 4.18 (broad s, 1H), $3.92(\mathrm{~s}, 3 \mathrm{H}), 3.73(\mathrm{~s}, 5 \mathrm{H})$.

${ }^{13} \mathrm{C}$ NMR $\left(126 \mathrm{MHz}, \mathrm{CDCl}_{3}\right): \delta 157.5,133.2,128.4,128.1,124.9,124.6,115.2,111.0,83.8,83.0,69.2$ (5C), 68.8, 63.7, 60.6, 55.4.

MS (EI): m/z (\%) $316\left(M^{+}, 100\right), 273(52), 215$ (47),

HRMS: Calculated for $\mathrm{C}_{19} \mathrm{H}_{16} \mathrm{FeO}\left(\mathrm{M}^{+}\right)$316.0551, found 316.0543 .

HPLC: Daicel Chiralpak IC, hexane / 2-propanol 97:3; $0.3 \mathrm{~mL} \mathrm{~min}^{-1}, 254 \mathrm{~nm}, R_{\mathrm{t}}=32.1 \mathrm{~min}, T=25 \stackrel{\circ}{ }$ ) .
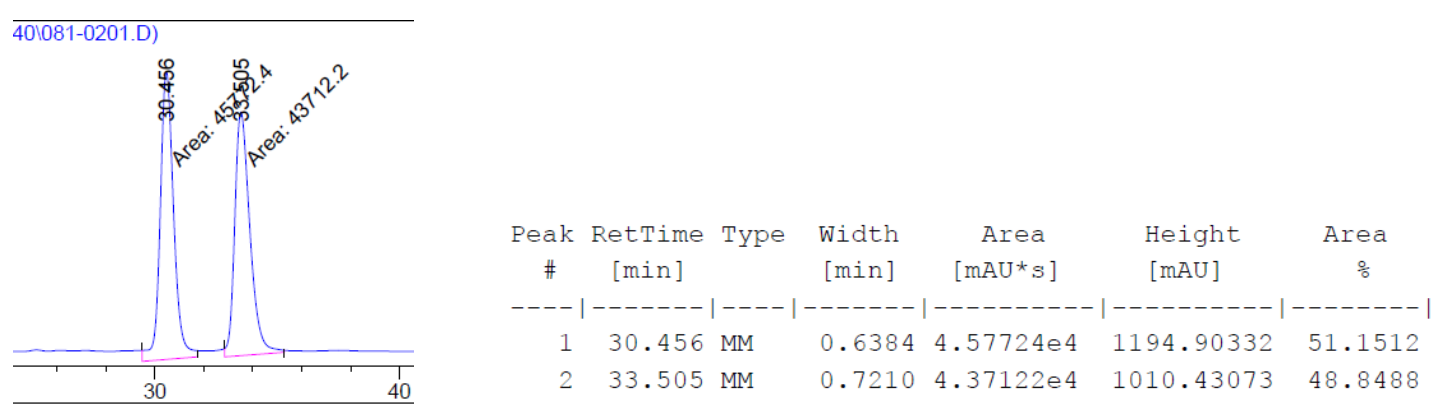

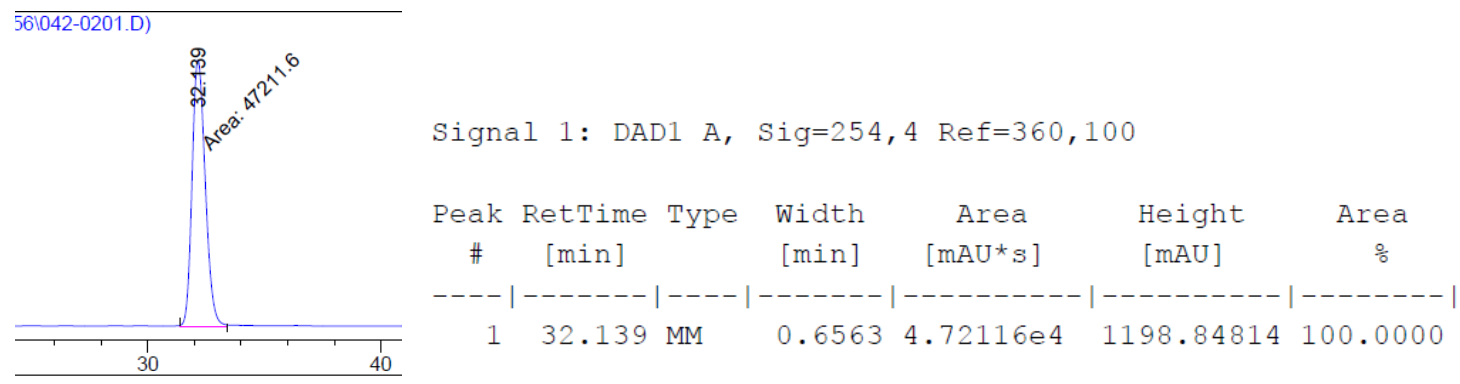

$\left(S_{S}, S_{p}\right)$-2-(2-Bromophenyl-1-(p-tolylsulfinyl)ferrocene (9d)

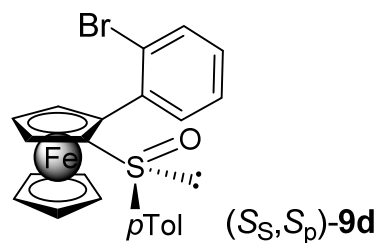

Compound $\left(S_{S}, S_{p}\right)$-9d was obtained from commercially available 2-bromoyodobenzene (8d) following general procedure $\boldsymbol{A}$. (eluent heptane/EtOAc 70:30), in $67 \%$ yield $(0.41 \mathrm{mmol}, 200 \mathrm{mg}$ ), as an orange solid. The physical and spectroscopic data agreed with those published in the literature. ${ }^{5}$

${ }^{1} \mathrm{H}$ NMR $\delta\left(300 \mathrm{MHz}, \mathrm{CDCl}_{3}\right): 8.35(\mathrm{~d}, J=7.2 \mathrm{~Hz}, 1 \mathrm{H}), 7.52(\mathrm{~m}, 2 \mathrm{H}), 7.45(\mathrm{~m}, 2 \mathrm{H}), 7.26(\mathrm{~m}, 3 \mathrm{H}), 4.85(\mathrm{bs}$, $1 \mathrm{H}), 4.70-4.60(\mathrm{~m}, 2 \mathrm{H}), 4.52(\mathrm{~s}, 5 \mathrm{H}), 2.49(\mathrm{~s}, 3 \mathrm{H})$.

$\left(S_{\mathrm{p}}\right)$-2-(2-Bromophenyl)-1-formylferrocene (10d).

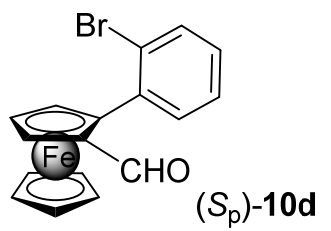

To a stirred solution of PhLi 1.8M solution in dibutyl ether $(4.27 \mathrm{~mL}, 7.69 \mathrm{mmol})$, under argon, a solution of sulfoxide $\left(S_{S}, S_{p}\right)-9 d(1.226 \mathrm{~g}, 2.56 \mathrm{mmol})$ in $18 \mathrm{~mL}$ of THF was added dropwise over $15 \mathrm{~min}$ at -78 o $\mathrm{C}$. The solution was stirred for $10 \mathrm{~min}$ at the same temperature and dry DMF (1.96 mL, $25.47 \mathrm{mmol}$ ) was

${ }^{5}$ Jensen, J. F.; Sotofte, I.; Sorensen, H. O.; Johannsen, M. J. Org. Chem. 2003, 68, 1258. 
added. The reaction mixture was stirred for $50 \mathrm{~min}$ at $-78 \stackrel{\circ}{\circ} \mathrm{C}$, warmed to room temperature and stirred for $1 \mathrm{~h}$. After quenching with saturated $\mathrm{NH}_{4} \mathrm{Cl}$, the mixture was extracted with $\mathrm{CH}_{2} \mathrm{Cl}_{2}$ and washed with brine. The residue was purified by flash chromatography (heptane/EtOAc 85:15) to afford pure aldehyde $\left(S_{\mathrm{p}}\right)-\mathbf{1 0 d}$ in $73 \%$ yield $(0.69 \mathrm{mmol}, 253 \mathrm{mg})$, as orange oil.

$[\alpha]_{D}^{20}=-560\left(c=0.005, \mathrm{CH}_{2} \mathrm{Cl}_{2}\right)$.

${ }^{1} \mathrm{H}$ NMR $\left(300 \mathrm{MHz}, \mathrm{CDCl}_{3}\right): \delta 9.88(\mathrm{~s}, 1 \mathrm{H}), 7.91(\mathrm{dd}, J=7.7,1.3 \mathrm{~Hz}, 1 \mathrm{H}), 7.57(\mathrm{~d}, J=8.0 \mathrm{~Hz}, 1 \mathrm{H}), 7.38(\mathrm{t}, J=$ $7.3 \mathrm{~Hz}, 1 \mathrm{H}), 7.19(\mathrm{t}, J=7.7 \mathrm{~Hz}, 1 \mathrm{H}), 5.04($ broad s, 1H), 4.75 (broad s, 1H), 4.71 (broad s, 1H), $4.33(\mathrm{~s}, 5 \mathrm{H})$.

${ }^{13} \mathrm{C}$ NMR (75 MHz, CDCl 3 ): $\delta$ 192.6, 135.6, 133.0, 132.1, 128.9, 127.4, 125.1, 92.6, 77.3, 76.5, 71.4, 70.9 (5C), 67.3.

MS (EI): m/z (\%) $368\left(M^{+}, 15\right), 183(100)$.

HMRS: Calculated for $\mathrm{C}_{17} \mathrm{H}_{13} \mathrm{FeO}\left(\mathrm{M}^{+}\right)$367.9501, found 367.9501.

\section{$\left(R_{\mathrm{p}}\right)$-2-(2-Bromophenyl)-1-ethynyl ferrocene (6d)}

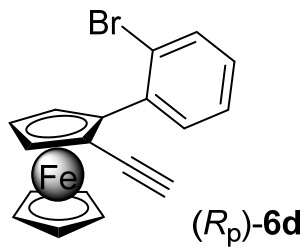

Compound $\left(R_{\mathrm{p}}\right)$-6d was obtained from $\left(S_{\mathrm{p}}\right)$-2-(2-bromophenyl)-1-formylferrocene (9d) following general procedure $\boldsymbol{D}$ (eluent heptane/EtOAc $95: 5$ ) in $55 \%$ yield $(0.2 \mathrm{mmol}, 72 \mathrm{mg}$ ), as an orange oil.

$[\alpha]_{D}=-27.2\left(c=0.06, \mathrm{CH}_{2} \mathrm{Cl}_{2}\right)$

${ }^{1} \mathrm{H}$ NMR $\left(300 \mathrm{MHz}, \mathrm{CDCl}_{3}\right): \delta 8.14(\mathrm{dd}, J=7.8,1.7 \mathrm{~Hz}, 1 \mathrm{H}), 7.57(\mathrm{dd}, J=8.0,1.3 \mathrm{~Hz}, 1 \mathrm{H}), 7.36(\mathrm{td}, J=7.6$, $1.3 \mathrm{~Hz}, 1 \mathrm{H}$ ), 7.14 (ddd, $J=8.0,7.3,1.7 \mathrm{~Hz}, 1 \mathrm{H}$ ), 4.72 (broad s, 1H), 4.64 (broad s, 1H), 4.37 (broad s, 1H), $4.25(\mathrm{~s}, 5 \mathrm{H}), 2.74(\mathrm{~s}, 1 \mathrm{H})$.

${ }^{13} \mathrm{C}$ NMR (126 MHz, $\left.\mathrm{CDCl}_{3}\right): \delta$ 136.7, 133.7, 132.9, 128.4, 126.5, 124.4, 90.2, 81.8, 76.0, 71.7, 71.5, 71.4 (5C), 67.6, 65.7. 
MS (EI): $m / z(\%) 364\left(M^{+}, 100\right), 366\left(M^{+}+2,98\right)$

HMRS: Calculated for $\mathrm{C}_{18} \mathrm{H}_{13} \mathrm{FeBr}\left(\mathrm{M}^{+}\right)$363.9550, found 363.9542 .

\section{$\left(R_{\mathrm{p}}\right)$-9-bromo-ferrocene-[3]-helicene (1d).}

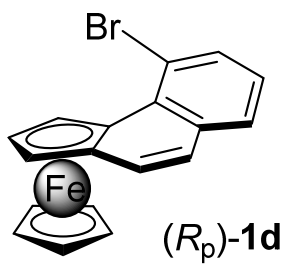

Compound $\left(\boldsymbol{R}_{p}\right)$-1d was obtained from 2-(2-bromophenyl-1-ethynyl-ferrocene (6d) following general procedure $E(2 \mathrm{~h})$, in $95 \%$ yield $(0.14 \mathrm{mmol}, 52 \mathrm{mg})$, as a red solid.

$[\alpha]_{D}=+970\left(c=0.015, \mathrm{CH}_{2} \mathrm{Cl}_{2}\right),>98 \%$ ee.

${ }^{1} \mathrm{H}$ NMR $\left(300 \mathrm{MHz}, \mathrm{CDCl}_{3}\right): 7.73(\mathrm{dd}, J=7.7,1.3 \mathrm{~Hz}, 1 \mathrm{H}), 7.56(\mathrm{dd}, J=7.6,1.3 \mathrm{~Hz}, 1 \mathrm{H}), 7.41(\mathrm{~d}, J=9.1 \mathrm{~Hz}$, $1 \mathrm{H}), 7.22(\mathrm{t}, J=7.8 \mathrm{~Hz}, 1 \mathrm{H}), 7.10(\mathrm{~d}, J=9.1 \mathrm{~Hz}, 1 \mathrm{H}), 6.31$ (broad s, $1 \mathrm{H}), 4.85$ (broad s, $1 \mathrm{H}), 4.28(\mathrm{t}, J=2.7$ $\mathrm{Hz}, 1 \mathrm{H}), 3.82(\mathrm{~s}, 5 \mathrm{H})$.

${ }^{13} \mathrm{C}$ NMR (126 MHz, CDCl $): \delta 135.1,134.8,132.7,128.9,128.5,125.7,125.4,120.0,85.0,80.9,70.0$, $69.4(5 \mathrm{C}), 66.8,64.8$.

MS (EI): $m / z(\%) 364\left(M^{+}, 100\right), 366\left(M^{+}+2,98\right)$.

HMRS: Calculated for $\mathrm{C}_{18} \mathrm{H}_{13} \mathrm{FeBr}\left(\mathrm{M}^{+}\right) 363.9550$, found 363.9551 .

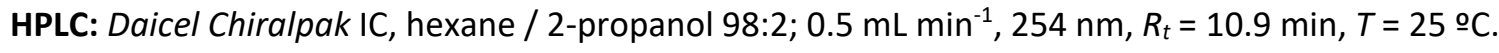
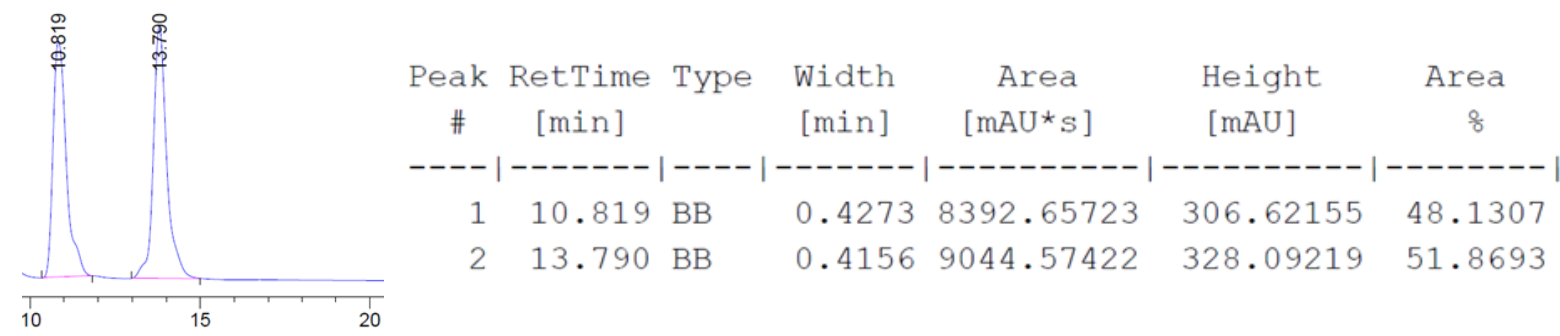

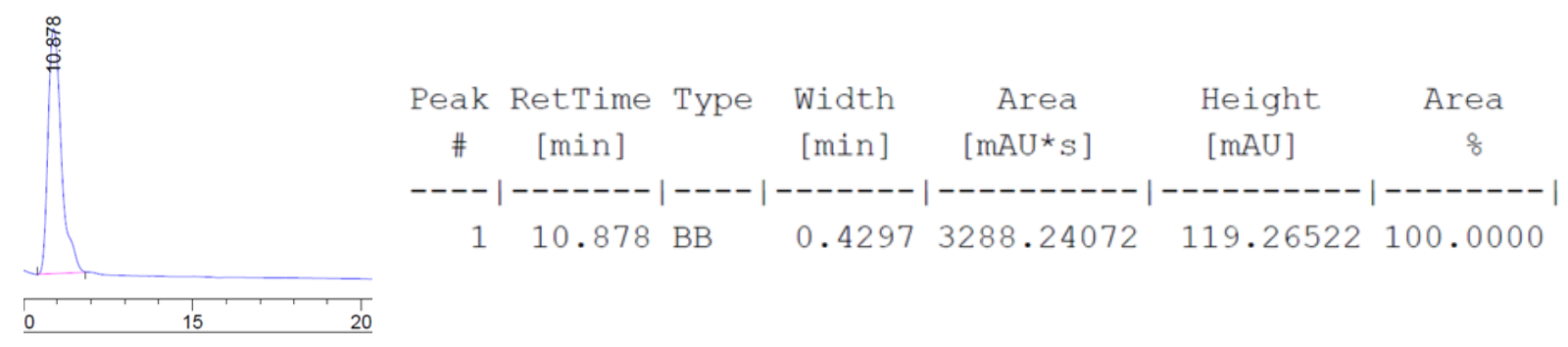

$\left(S_{\mathrm{s}}, S_{\mathrm{p}}\right)$-2-Napththyl-1-(p-tolylsulfinyl)ferrocene $(9 \mathrm{e})$.

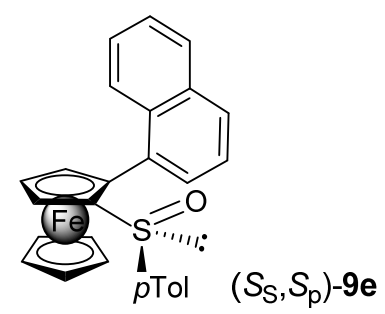

Compound $\left(S_{S}, S_{p}\right)-9 e$ was obtained from commercially available 1-iodonapthalene (8e) following general procedure $\boldsymbol{A}$ (eluent $\mathrm{CH}_{2} \mathrm{Cl}_{2}:$ EtOAc $\left.95: 5\right)$ in $72 \%$ yield $(0.45 \mathrm{mmol}, 200 \mathrm{mg}$ ), as an orange solid. The physical and spectroscopic data agreed with those published in the literature. ${ }^{6}$

${ }^{1} \mathrm{H}$ NMR $\delta\left(300 \mathrm{MHz}, \mathrm{CDCl}_{3}\right): 8.24(\mathrm{~d}, J=6.8 \mathrm{~Hz}, 1 \mathrm{H}), 7.80(\mathrm{dd}, J=12.6,8.5 \mathrm{~Hz}, 2 \mathrm{H}), 7.57(\mathrm{t}, J=7.5 \mathrm{~Hz}, 1 \mathrm{H})$, $7.47(\mathrm{~d}, J=8.4 \mathrm{~Hz}, 1 \mathrm{H}), 7.41-7.18(\mathrm{~m}, 4 \mathrm{H}), 6.95(\mathrm{~d}, J=7.5 \mathrm{~Hz}, 2 \mathrm{H}), 4.65$ (broad s, $1 \mathrm{H}), 4.57$ (broad s, $1 \mathrm{H}$ ), $4.51($ broad s, $1 \mathrm{H}), 4.41(\mathrm{~s}, 5 \mathrm{H}), 2.24(\mathrm{~s}, 3 \mathrm{H})$.

$\left(S_{\mathrm{p}}\right)$-1-Formyl-2-naphthylferrocene (10e).

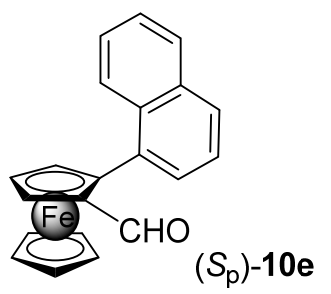

(5) Pedersen, H. L.; Johannsen, M. J. Org. Chem. 2002, 67, 7982. 
Compound $\left(S_{p}\right)$-10e was obtained from $\left(S_{s}, S_{p}\right)$-2-napththyl-1-( $p$-tolylsulfinyl)ferrocene $9 e$ following general procedure $\boldsymbol{B}$ (eluent hexane/EtOAc 5:1), in $95 \%$ yield $(0.89 \mathrm{mmol}, 304 \mathrm{mg}$ ), as an orange oil. The physical and spectroscopic data agreed with those published in the literature. ${ }^{7}$

${ }^{1} \mathrm{H}$ NMR $\delta\left(300 \mathrm{MHz}, \mathrm{CDCl}_{3}\right): 9.80(\mathrm{~s}, 1 \mathrm{H}), 8.07(\mathrm{~d}, J=7.0 \mathrm{~Hz}, 1 \mathrm{H}), 7.85(\mathrm{~m}, 3 \mathrm{H}), 7.55(\mathrm{t}, J=7.7 \mathrm{~Hz}, 1 \mathrm{H})$, $7.46(\mathrm{~m}, 2 \mathrm{H}), 5.15$ (broad s, 1H), $4.83(\mathrm{~m}, 2 \mathrm{H}), 4.39(\mathrm{~s}, 5 \mathrm{H})$.

$\left(R_{\mathrm{p}}\right)$-2-Ethynyl-1-naphthylferrocene (6e).

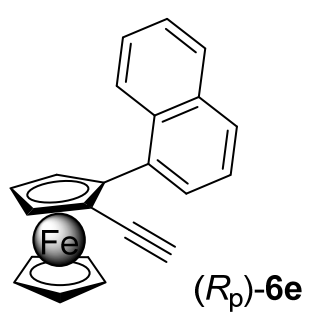

Compound $\left(R_{\mathrm{p}}\right)$-6e was obtained from $\left(S_{\mathrm{p}}\right)$-1-formyl-2-napthylferrocene 10e following general procedure C (eluent hexane), in $73 \%$ yield $(0.44 \mathrm{mmol}, 147 \mathrm{mg})$, as an orange foam.

$[\alpha]_{D}^{20}=-31\left(c=0.042, \mathrm{CH}_{2} \mathrm{Cl}_{2}\right)$.

${ }^{1} \mathrm{H}$ NMR $\delta\left(300 \mathrm{MHz}, \mathrm{CDCl}_{3}\right): 8.22(\mathrm{~d}, J=7.2 \mathrm{~Hz}, 1 \mathrm{H}), 8.12(\mathrm{~d}, J=8.9 \mathrm{~Hz}, 1 \mathrm{H}), 7.86(\mathrm{~m}, 2 \mathrm{H}), 7.56(\mathrm{t}, J=7.7$ $\mathrm{Hz}, 1 \mathrm{H}), 7.49-7.37(\mathrm{~m}, 2 \mathrm{H}), 4.72$ (broad s, 1H), 4.62 (broad s, 1H), 4.45 (broad s, 1H), 4.30 (s, 5H), 2.68 $(\mathrm{s}, 1 \mathrm{H})$.

${ }^{13} \mathrm{C}$ NMR $\delta$ (75 MHz, $\left.\mathrm{CDCl}_{3}\right): 133.6,133.4,132.5,129.6,128.3,127.6,126.1,125.5,125.5,124.9,90.3$, 82.0, 76.0, 71.9, 71.7, $71.5(5 \mathrm{C}), 67.8,66.5$.

MS (ESI+): $m / z(\%) 336\left(\mathrm{M}^{+}, 100\right)$

HRMS: Calculated for $\mathrm{C}_{22} \mathrm{H}_{16} \mathrm{Fe}\left(\mathrm{M}^{+}\right)$336.0601, found 336.0606.

(6) Mamane, V.; Riant, O. Tetrahedron 2001, 57, 2555. 
$\left(\boldsymbol{R}_{\mathrm{p}}\right)$-Ferrocene-[4]helicene (12).

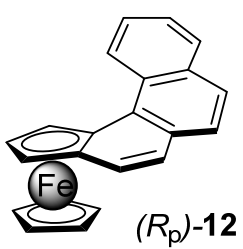

Compound $\left(R_{\mathrm{p}}\right)$-12 was obtained from $\left(R_{\mathrm{P}}\right)$-2-ethynyl-1-naphthylferrocene $(6 \mathrm{e})$ following general procedure $E(5 \mathrm{~h})$, in $92 \%$ yield $(0.14 \mathrm{mmol}, 46 \mathrm{mg})$.

$[\alpha]_{D}^{20}=+2270\left(c 0.014, \mathrm{CH}_{2} \mathrm{Cl}_{2}\right), 98 \%$ ee.

${ }^{1} \mathrm{H}$ NMR $\delta\left(500 \mathrm{MHz}, \mathrm{CDCl}_{3}\right): 9.17(\mathrm{~d}, J=8.6 \mathrm{~Hz}, 1 \mathrm{H}), 8.01(\mathrm{~d}, J=8.0 \mathrm{~Hz}, 1 \mathrm{H}), 7.85(\mathrm{~d}, J=8.4 \mathrm{~Hz}, 1 \mathrm{H}), 7.78$ (t, $J=7.7 \mathrm{~Hz}, 1 \mathrm{H}), 7.71(\mathrm{~d}, J=8.4 \mathrm{~Hz}, 1 \mathrm{H}), 7.63(\mathrm{~m}, 2 \mathrm{H}), 7.37(\mathrm{~d}, J=8.9 \mathrm{~Hz}, 1 \mathrm{H}), 5.79$ (broad s, 1H), 5.03 (broad s, 1H), 4.34 (broad s, 1H), $3.76(\mathrm{~s}, 5 \mathrm{H})$.

${ }^{13} \mathrm{C}$ NMR $\delta$ (126 MHz, CDCl $)$ ): 133.2, 131.9, 131.1, 130.9, 128.8, 128.5, 127.9, 126.3, 126.1, 126.1, 125.7, $125.5,86.7,82.3,70.8,68.9(5 C), 65.6,64.0$.

MS (EI): $m / z(\%) 336\left(\mathrm{M}^{+}, 50\right), 154$ (88), 57 (100).

HRMS: Calculated for $\mathrm{C}_{22} \mathrm{H}_{16} \mathrm{Fe}\left(\mathrm{M}^{+}\right) 336.0601$, found 336.0596 .

HPLC: Daicel Chiralpak IB, hexano / 2-propanol 98:2; $0.5 \mathrm{~mL} \mathrm{~min}^{-1}, 254 \mathrm{~nm}, R_{t}=17.3 \mathrm{~min}, T=25^{\circ} \mathrm{C}$ ).
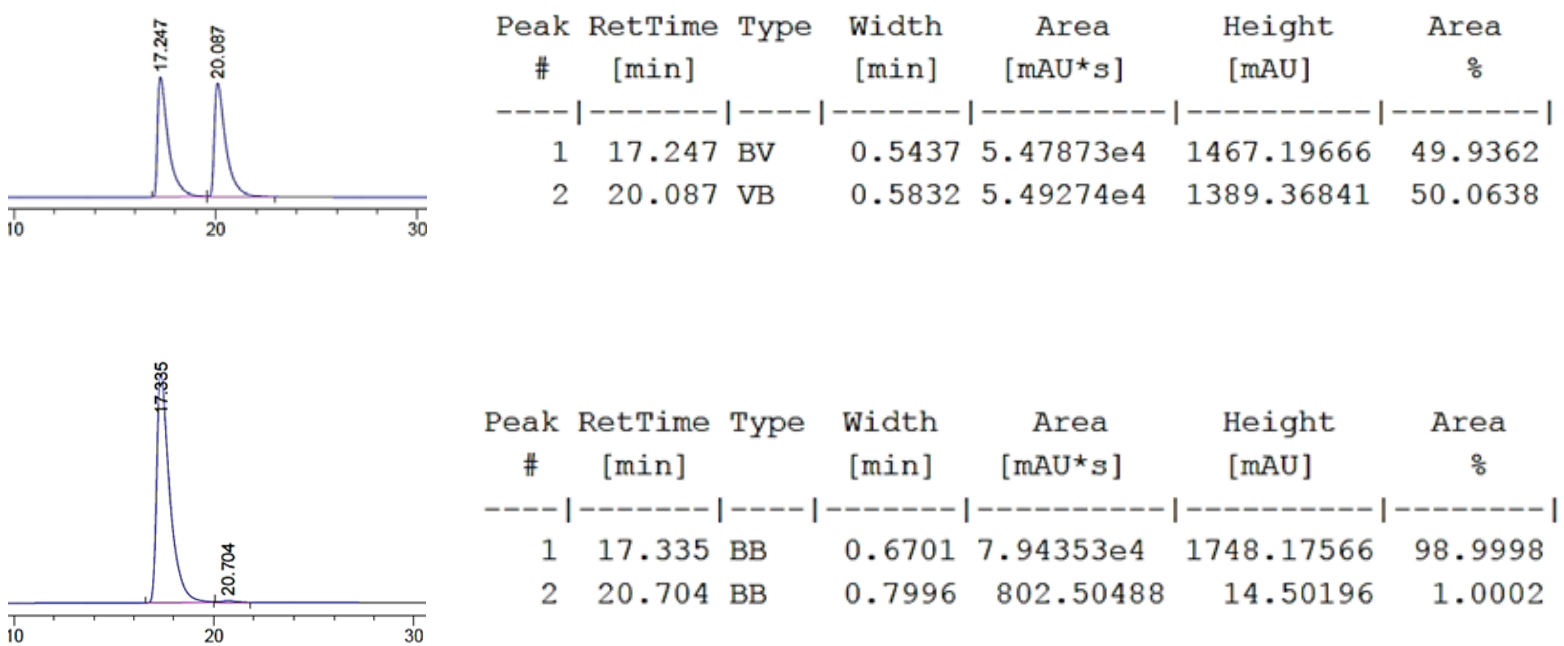
$\left(S_{\mathrm{p}}\right)-2-\left\{\left[1,1^{\prime}\right.\right.$-Biphenyl]-2-carbaldehyde\}-1-formylferrocene (14).

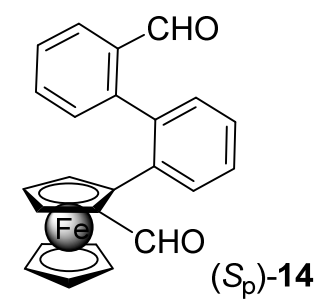

To a mixture of aryl bromide $\left(S_{\mathrm{P}}\right)-\mathbf{1 0 d}(142 \mathrm{mg}, 0.38 \mathrm{mmol})$, commercially available 2-formylphenyl boronic acid (13) (116 mg, $0.77 \mathrm{mmol}$ ) and $\mathrm{Pd}\left(\mathrm{PPh}_{3}\right)_{4}(43 \mathrm{mg}, 0.039 \mathrm{mmol})$, under argon, DME (2.5 mL) and aq. $2 \mathrm{M} \mathrm{Na}_{2} \mathrm{CO}_{3}$ solution $(0.41 \mathrm{~mL}, 0.770 \mathrm{mmol})$ were added. The mixture was heated at $110{ }^{\circ} \mathrm{C}$ for 6 h. After workup, the residue was purified by flash chromatography (eluent hexane/EtOAc 6:1) affording pure bis-aldehyde $\left(S_{\mathrm{P}}\right)-\mathbf{1 4}$ (mixture of atropisomers), as an orange foam, in 64\% yield $(0.24 \mathrm{mmol}, 96$ $\mathrm{mg})$.

$[\alpha]_{D}{ }^{20}=-331\left(c=0.016, \mathrm{CH}_{2} \mathrm{Cl}_{2}\right)$.

Major atropoisomer ${ }^{1} \mathrm{H}$ NMR $\delta\left(300 \mathrm{MHz}, \mathrm{CDCl}_{3}\right): 9.51(\mathrm{~s}, 1 \mathrm{H}), 9.45(\mathrm{~s}, 1 \mathrm{H}), 8.00(\mathrm{~d}, J=7.7 \mathrm{~Hz}, 1 \mathrm{H}), 7.72$ $(\mathrm{d}, J=7.3 \mathrm{~Hz}, 1 \mathrm{H}), 7.57-7.49(\mathrm{~m}, 2 \mathrm{H}), 7.46(\mathrm{~d}, J=8.3 \mathrm{~Hz}, 1 \mathrm{H}), 7.36(\mathrm{t}, J=6.8 \mathrm{~Hz}, 3 \mathrm{H}), 4.68$ (broad s, $1 \mathrm{H}$ ), $4.53(\mathrm{~m}, 2 \mathrm{H}), 4.26(\mathrm{~s}, 5 \mathrm{H})$. Minor atropoisomer ${ }^{1} \mathrm{H}$ NMR $\delta\left(300 \mathrm{MHz}, \mathrm{CDCl}_{3}\right)$ : $9.77(\mathrm{~s}, 1 \mathrm{H}), 9.73(\mathrm{~s}, 1 \mathrm{H})$, $8.04(\mathrm{~d}, J=6.8 \mathrm{~Hz}, 1 \mathrm{H}) ,7.82(\mathrm{~d}, J=8.7 \mathrm{~Hz}, 1 \mathrm{H}), 7.25(\mathrm{~d}, J=4.4 \mathrm{~Hz}, 5 \mathrm{H}), 6.99(\mathrm{~d}, J=6.0 \mathrm{~Hz}, 1 \mathrm{H}), 4.72$ (broad s, 1H), 4.58 (broad s, 1H), 4.37 (broad s, 1H), $4.27(\mathrm{~s}, 5 \mathrm{H})$.

${ }^{13} \mathrm{C}$ NMR $\delta$ (75 MHz, CDCl $): 191.9,191.5,190.8,190.7,171.0,144.2,144.10,138.5,138.3,135.4,134.8$, 133.9, 133.78, 133.3, 132.8, 132.6, 131.9, 131.5, 131.3, 131.2, 130.2, 128.3, 128.1, 127-9, 127.8, 127.6, $127.2,126.9,92.1,91.1,77.2,76.6,76.5,71.7,71.4,71.4,70.9,68.7,67.4$.

MS (FAB+): $m / z(\%) 394\left(\mathbf{M}^{+}, 4\right), 57(100)$.

HRMS: Calculated for $\mathrm{C}_{24} \mathrm{H}_{18} \mathrm{FeO}\left(\mathrm{M}^{+}\right)$394.0656, found 394.0659. 
$\left(R_{P}\right)$-1-\{2-Ethynyl-[1,1'-biphenyl]\}-2-ethynylferrocene (15).

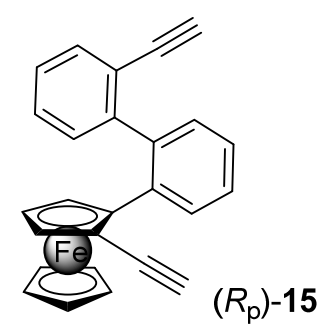

Compound $\left(R_{\mathrm{p}}\right)-15$ was obtained, as a mixture of atropisomers, from $\left(S_{\mathrm{p}}\right)-2-\left\{\left[1,1^{\prime}\right.\right.$-biphenyl]-2carbaldehyde\}-1-formylferrocene (14) following general procedure $D$ (eluent hexane) in $60 \%$ yield $(0.22$ $\mathrm{mmol}, 83 \mathrm{mg})$, as an orange foam.

$[\alpha]_{D}{ }^{20}=+67\left(c=0.03, \mathrm{CH}_{2} \mathrm{Cl}_{2}\right)$.

${ }^{1} \mathrm{H}$ NMR $\delta\left(300 \mathrm{MHz}, \mathrm{CDCl}_{3}\right):$ (mixture of atropisomers) $8.38-8.19(\mathrm{~m}, 1 \mathrm{H}), 7.59-7.43(\mathrm{~m}, 1 \mathrm{H}), 7.35(\mathrm{~s}$, 3H), $7.04(\mathrm{~m}, 1 \mathrm{H}), 6.74-6.71(\mathrm{~m}, 1 \mathrm{H}), 4.48(\mathrm{~s}, 1 \mathrm{H}), 4.14(\mathrm{~s}, 5 \mathrm{H}), 4.00$ (bs, $1 \mathrm{H}$, two peaks due to chiral axis), $3.06(\mathrm{~s}, 0.5 \mathrm{H}$, minor diasteroisomer), 2.83-2.77 ( $\mathrm{s}, 1.5 \mathrm{H}$, three peaks due to chiral axis).

${ }^{13} \mathrm{C}$ NMR $\delta\left(75 \mathrm{MHz}, \mathrm{CDCl}_{3}\right): 144.9,140.1,135.8,134.8,132.9,132.5,132.2,131.9,130.9,130.5,130.1$, $129.9,128.2,128.0,126.9,126.7,126.6,126.1,125.5,122.2,121.5,82.5,80.1,71.3,70.6,70.3,67.5$, $67.3,66.2,65.6,60.4$.

MS (FAB+): $m / z(\%) 386\left(\mathbf{M}^{+}, 95\right), 265$ (100).

HRMS: Calculated for $\mathrm{C}_{26} \mathrm{H}_{18} \mathrm{Fe}\left(\mathrm{M}^{+}\right)$386.0758, found 386.0753 .

\section{2'-Bromo-[1,1'-biphenyl]-2-carbaldehyde (18).}<smiles>O=Cc1ccccc1-c1ccccc1Br</smiles>

To a mixture of commercially available 2-bromophenyl boronic acid (260 mg, $1.3 \mathrm{mmol}$ ) and $\mathrm{Pd}\left(\mathrm{PPh}_{3}\right)_{4}$ (125 mg, $0.108 \mathrm{mmol}$ ), under argon, commercially available 2-bromobenzaldehyde (126 $\mu \mathrm{L}, 1.08 \mathrm{mmol}$ ), $\mathrm{Et}_{3} \mathrm{~N}(0.45 \mathrm{~mL}, 3.24 \mathrm{mmol})$ and $\mathrm{DMF}(5.4 \mathrm{~mL})$ were added. The mixture was heated at $90^{\circ} \mathrm{C}$ for $2 \mathrm{~h}$. After 
workup and solvent elimination, the residue was purified by flash chromatography (eluent hexane/EtOAc 7:1) affording pure aldehyde 18, in $82 \%$ yield $(0.89 \mathrm{mmol}, 230 \mathrm{mg})$, as colorless oil. The physical and spectroscopic data agreed with those published in the literature. ${ }^{8}$

${ }^{1} \mathrm{H}$ NMR $\delta\left(300 \mathrm{MHz}, \mathrm{CDCl}_{3}\right): 9.80(\mathrm{~s}, 1 \mathrm{H}), 8.04(\mathrm{~d}, J=7.7 \mathrm{~Hz}, 1 \mathrm{H}), 7.74-7.66(\mathrm{~m}, 2 \mathrm{H}), 7.55(\mathrm{t}, J=7.4 \mathrm{~Hz}$, $1 \mathrm{H}), 7.41(\mathrm{~m}, 1 \mathrm{H}), 7.32(\mathrm{~m}, 3 \mathrm{H})$.

\section{2-Bromo-2'-ethynyl-1,1'-biphenyl (19).}<smiles>C#Cc1ccccc1-c1ccccc1Br</smiles>

Compound 19 was obtained from 2'-bromo-[1,1'-biphenyl]-2-carbaldehyde (18) following general procedure $\boldsymbol{D}$ (eluent hexane) in $85 \%$ yield $(0.31 \mathrm{mmol}, 79 \mathrm{mg})$, as a colorless oil.

${ }^{1} \mathrm{H}$ NMR $\delta\left(300 \mathrm{MHz}, \mathrm{CDCl}_{3}\right): 7.71(\mathrm{~d}, J=7.9 \mathrm{~Hz}, 1 \mathrm{H}), 7.66(\mathrm{~d}, J=7.3 \mathrm{~Hz}, 1 \mathrm{H}), 7.41(\mathrm{~m}, 5 \mathrm{H}), 7.32(\mathrm{~m}, 1 \mathrm{H})$, $2.99(\mathrm{~s}, 1 \mathrm{H})$.

${ }^{13} \mathrm{C}$ NMR $\delta\left(75 \mathrm{MHz}, \mathrm{CDCl}_{3}\right): 144.0,141.2,132.8,132.5,131.1,129.7,129.0,128.3,127.6,126.8,123.2$, 121.6, 82.0, 80.3.

MS (EI): $m / z(\%) 258\left(\mathrm{M}^{+}+2,32\right), 256\left(\mathrm{M}^{+}, 30\right), 177(100)$.

HRMS: Calculated for $\mathrm{C}_{14} \mathrm{H}_{9} \mathrm{Br}\left(\mathrm{M}^{+}\right) 255.9888$, found 255.9884 .

\section{4-Bromophenathrene (8f).}<smiles>Brc1cccc2ccc3ccccc3c12</smiles>

(7) Paul, S.; Samanta, S.; Ray, J. K. Tetrahedron Lett. 2010, 51, 5604. 
To a mixture of alkyne 19 (231 mg, $0.9 \mathrm{mmol}$ ) and $\mathrm{PtCl}_{2}$ (18 mg, $0.072 \mathrm{mmol}$ ), under argon, dry DCE (5 $\mathrm{mL}$ ) was added and the mixture heated at $100{ }^{\circ} \mathrm{C}$ for $24 \mathrm{~h}$. The crude mixture was filtered through celite and washed with hexane. After solvent removal, pure phenantrene $8 \mathrm{f}$ was obtained, in $92 \%$ yield $(0.83$ $\mathrm{mmol}, 213 \mathrm{mg}$ ), as a brown oil. The physical and spectroscopic data agreed with those published in the

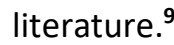

${ }^{1} \mathrm{H}$ NMR $\delta\left(300 \mathrm{MHz}, \mathrm{CDCl}_{3}\right): 10.07(\mathrm{~d}, J=9.0 \mathrm{~Hz}, 1 \mathrm{H}), 8.01(\mathrm{~d}, J=7.6 \mathrm{~Hz}, 1 \mathrm{H}), 7.94-7.87(\mathrm{~m}, 1 \mathrm{H}), 7.85(\mathrm{~d}$, $J=7.8 \mathrm{~Hz}, 1 \mathrm{H}), 7.75(\mathrm{~d}, J=8.8 \mathrm{~Hz}, 1 \mathrm{H}), 7.69-7.64(\mathrm{~m}, 3 \mathrm{H}), 7.38(\mathrm{t}, J=7.7 \mathrm{~Hz}, 1 \mathrm{H})$.

$\left(S_{s,} S_{\mathrm{p}}\right)$-2-Phenanthrenyl-1-( $p$-tolylsulfinyl)ferrocene (9f).

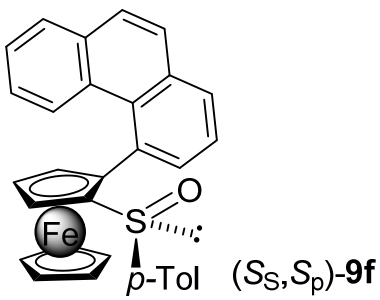

Compound $\left(S_{\mathrm{S}}, S_{\mathrm{p}}\right)$-9f was obtained (as a mixture of atropisomers) from 4-bromophenanthrene (8f) following general procedure $\boldsymbol{A}$ (eluent hexane:EtOAc 3:1), in $96 \%$ yield $(0.59 \mathrm{mmol}, 300 \mathrm{mg}$ ), as an orange solid.

$[\alpha]_{D}^{20}=-120\left(c=0.070, \mathrm{CH}_{2} \mathrm{Cl}_{2}\right)$.

Major atropisomer: ${ }^{1} \mathrm{H}$ NMR $\delta\left(300 \mathrm{MHz}, \mathrm{CDCl}_{3}\right): 8.60(\mathrm{~d}, J=6.9 \mathrm{~Hz}, 1 \mathrm{H}), 7.86(\mathrm{~d}, J=5.3 \mathrm{~Hz}, 1 \mathrm{H}), 7.67(\mathrm{~m}$, $4 \mathrm{H}), 7.46(\mathrm{~d}, J=7.8 \mathrm{~Hz}, 2 \mathrm{H}), 7.36(\mathrm{t}, J=7.3 \mathrm{~Hz}, 1 \mathrm{H}), 7.21(\mathrm{~d}, J=8.6 \mathrm{~Hz}, 1 \mathrm{H}), 7.00(\mathrm{~m}, 3 \mathrm{H}), 4.60$ (broad s, 1H), 4.48 (broad s, 1H), 4.37 (broad s, 1H), $4.29(\mathrm{~s}, 5 \mathrm{H}), 2.18(\mathrm{~s}, 3 \mathrm{H})$.

Minor atropisomer: ${ }^{1} \mathrm{H}$ NMR $\delta\left(300 \mathrm{MHz}, \mathrm{CDCl}_{3}\right): 8.67(\mathrm{~d}, J=6.3 \mathrm{~Hz}, 1 \mathrm{H}), 7.89(\mathrm{~d}, J=7.0 \mathrm{~Hz}, 1 \mathrm{H}), 7.72-$ $7.67(\mathrm{~m}, 3 \mathrm{H}), 7.63(\mathrm{dd}, J=8.5,6.5 \mathrm{~Hz}, 2 \mathrm{H}), 6.99(\mathrm{~d}, J=8.1 \mathrm{~Hz}, 2 \mathrm{H}), 6.77-6.71(\mathrm{~m}, 2 \mathrm{H}), 6.66(\mathrm{~d}, J=8.0 \mathrm{~Hz}$, 2H), 5.03 (broad s, 1H), $4.53(\mathrm{~s}, 5 \mathrm{H}), 4.33$ (broad s, 1H), 4.29 (broad s, 1H), $2.03(\mathrm{~s}, 3 \mathrm{H})$.

${ }^{13} \mathrm{C}$ NMR $\delta\left(75 \mathrm{MHz}, \mathrm{CDCl}_{3}\right.$ ): 140.6, 135.4, 134.5, 133.1, 133.0, 132.9, 131.2, 131.0, 129.7, 129.6, 129.0, 128.9, 128.8, 128.6, 128.6, 127.8, 127.7, 127.6, 127.3, 127.1, 127.0, 126.9, 125.7, 125.1, 124.8, 124.5, 124.1, 123.9, 123.5, 95.4, 94.9, 74.1, 73.4, 71.4, 71.2, 68.6, 68.3, 68.0, 63.4, 21.1, 20.9.

(8) Newman, M. S.; Patrick, T. B.; Darlak, R. S.; Zuech, E. A. J. Org. Chem. 1969, 34, 1904. 
MS (FAB+): $m / z(\%) 500\left(\mathbf{M}^{+}, 25\right), 57(97)$.

HRMS: Calculated for $\mathrm{C}_{31} \mathrm{H}_{24} \mathrm{FeOS}\left(\mathrm{M}^{+}\right) 500.0897$, found 500.0889 .

$\left(S_{\mathrm{p}}\right)$-1-Formyl-2-phenanthrenylferrocene (10f).

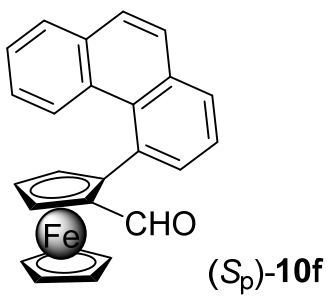

Compound $\left(S_{p}\right)$-10f was obtained from $\left(S_{S}, S_{p}\right)$-2-phenanthrenyl-1-( $p$-tolylsulfinyl)ferrocene (9f) following general procedure $\boldsymbol{B}$ (eluent hexane/EtOAc 5:1), in $75 \%$ yield $(0.71 \mathrm{mmol}, 275 \mathrm{mg}$ ), as an orange oil.

$[\alpha]_{D}{ }^{20}=-420\left(c=0.020, \mathrm{CH}_{2} \mathrm{Cl}_{2}\right)$.

${ }^{1} \mathrm{H}$ NMR $\delta\left(300 \mathrm{MHz}, \mathrm{CDCl}_{3}\right): 9.47(\mathrm{~s}, 1 \mathrm{H}), 8.41(\mathrm{~d}, J=7.2 \mathrm{~Hz}, 1 \mathrm{H}), 7.87(\mathrm{~d}, J=7.8 \mathrm{~Hz}, 1 \mathrm{H}), 7.77(\mathrm{~d}, J=7.8$ $\mathrm{Hz}, 1 \mathrm{H}), 7.74-7.59(\mathrm{~m}, 3 \mathrm{H}), 7.41(\mathrm{dd}, J=15.9,8.3 \mathrm{~Hz}, 2 \mathrm{H}), 7.11(\mathrm{t}, J=7.8 \mathrm{~Hz}, 1 \mathrm{H}), 5.13$ (broad s, $1 \mathrm{H}$ ), $4.93(\mathrm{~m}, 2 \mathrm{H}), 4.30(\mathrm{~s}, 5 \mathrm{H})$.

${ }^{13} \mathrm{C} \mathrm{NMR} \delta\left(75 \mathrm{MHz}, \mathrm{CDCl}_{3}\right): 193.3,133.8,133.5,133.0,131.8,131.0,129.2,128.6,128.4,128.3,127.6$, $126.9,126.2,125.1,124.5,98.5,76.2,71.8,71.4,71.2$ (5C), 67.1.

MS (EI): $m / z(\%) 390\left(\mathbf{M}^{+}, 100\right), 252$ (95).

HRMS: Calculated for $\mathrm{C}_{25} \mathrm{H}_{18} \mathrm{FeO}\left(\mathrm{M}^{+}\right) 390.0707$, found 390.0699 .

$\left(R_{\mathrm{p}}\right)$-2-Ethynyl-1-phenanthrenylferrocene (6f).

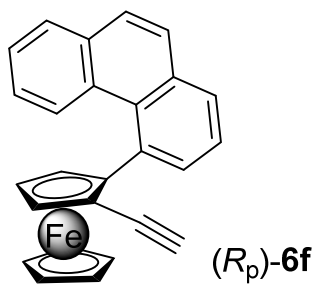


Compound $\left(R_{p}\right)$-6f was obtained from $\left(S_{p}\right)$-1-formyl-2-phenanthrenylferrocene (10f) following general procedure $\boldsymbol{C}$ (eluent hexane), in $52 \%$ yield $(0.31 \mathrm{mmol}, 120 \mathrm{mg})$, as an orange foam.

$[\alpha]_{D}^{20}=+93\left(c=0.015, \mathrm{CH}_{2} \mathrm{Cl}_{2}\right)$.

${ }^{1} \mathrm{H}$ NMR $\delta\left(300 \mathrm{MHz}, \mathrm{CDCl}_{3}\right): 8.51(\mathrm{~d}, J=7.2 \mathrm{~Hz}, 1 \mathrm{H}), 7.83(\mathrm{~d}, J=7.6 \mathrm{~Hz}, 1 \mathrm{H}), 7.77(\mathrm{~d}, J=7.9 \mathrm{~Hz}, 1 \mathrm{H}), 7.73$ $-7.62(\mathrm{~m}, 3 \mathrm{H}), 7.57(\mathrm{~d}, J=8.5 \mathrm{~Hz}, 1 \mathrm{H}), 7.41(\mathrm{t}, J=6.9 \mathrm{~Hz}, 1 \mathrm{H}), 7.12(\mathrm{t}, J=7.6 \mathrm{~Hz}, 1 \mathrm{H}), 4.79$ (broad s, 1H), $4.36(\mathrm{~m}, 2 \mathrm{H}), 4.25(\mathrm{~s}, 5 \mathrm{H}), 2.54(\mathrm{~s}, 1 \mathrm{H})$.

${ }^{13} \mathrm{C}$ NMR $\delta\left(75 \mathrm{MHz}, \mathrm{CDCl}_{3}\right): 137.3,137.1,133.7,133.2,133.1,132.9,130.3,128.1,127.9,127.2,127.0$, $125.8,124.9,124.1,96.8,81.6,76.6,75.6,71.8,71.5$ (5C), 67.8, 66.0.

MS (EI): $m / z(\%) 386\left(\mathrm{M}^{+}, 90\right), 264$ (100).

HRMS: Calculated for $\mathrm{C}_{26} \mathrm{H}_{18} \mathrm{Fe}\left(\mathrm{M}^{+}\right) 386.0758$, found 386.0749 .

$\left(R_{P}\right)$-Ferrocene-[5]helicene (5).

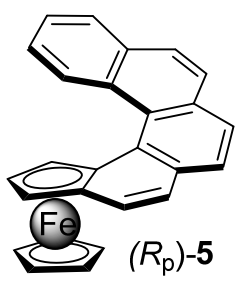

Compound $\left(R_{\mathrm{p}}\right)-5$ was obtained from 2-ethynyl-1-phenanthrenylferrocene (6f) following general procedure $E(5 \mathrm{~h})$, in $93 \%$ yield $(0.14 \mathrm{mmol}, 54 \mathrm{mg})$, as a red solid.

$[\alpha]_{D}^{20}=+3240\left(c=0.0025, \mathrm{CHCl}_{2}\right),>98 \%$ ee.

${ }^{1} \mathrm{H}$ NMR $\delta\left(300 \mathrm{MHz}, \mathrm{CDCl}_{3}\right): 8.75(\mathrm{~d}, J=8.6 \mathrm{~Hz}, 1 \mathrm{H}), 7.97(\mathrm{~d}, J=5.9 \mathrm{~Hz}, 1 \mathrm{H}), 7.84(\mathrm{~m}, 2 \mathrm{H}), 7.73(\mathrm{~m}, 3 \mathrm{H})$, $7.56(\mathrm{~m}, 1 \mathrm{H}), 7.33(\mathrm{~m}, 1 \mathrm{H}), 7.17(\mathrm{~m}, 1 \mathrm{H}), 5.34$ (broad s, 1H), 5.02 (broad s, 1H), 4.23 (broad s, 1H), 3.29 (s, $5 \mathrm{H})$.

${ }^{13} \mathrm{C}$ NMR $\delta$ (375 MHz, CDCl $): 133.1,132.8,131.9,131.8,130.0,129.4,128.9,127.5,127.4,127.0,126.7$, $126.1,125.7,125.1,124.7,123.5,86.7,85.9,70.7,69.6(5 C), 66.4,62.9$.

MS (EI): $m / z(\%) 386\left(\mathrm{M}^{+}, 25\right), 263(100)$. 
HRMS: Calculated for $\mathrm{C}_{26} \mathrm{H}_{18} \mathrm{Fe}\left(\mathrm{M}^{+}\right)$386.0758, found 386.0741 .

HPLC: Daicel Chiralpak IB, hexano / 2-propanol 97:3; $0.3 \mathrm{~mL} \mathrm{~min}^{-1}, 254 \mathrm{~nm}, R_{t}=22.3 \mathrm{~min}, T=25^{\circ} \mathrm{C}$ ).

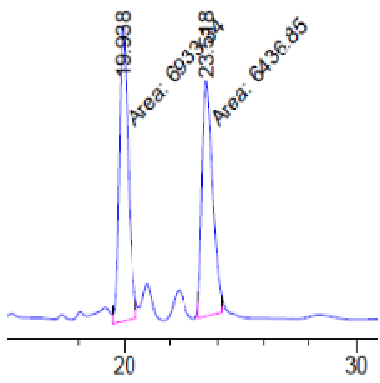

Signal 1: DAD1 A, Sig=254, 4 Ref $=360,100$

\begin{tabular}{|c|c|c|c|c|c|}
\hline $\begin{array}{c}\text { Peak } \\
\quad \#\end{array}$ & $\begin{array}{l}\text { RetTime Type } \\
\text { [min] }\end{array}$ & $\begin{array}{l}\text { Width } \\
\text { [min] }\end{array}$ & $\begin{array}{c}\text { Area } \\
{[\mathrm{mAU} * \mathrm{~S}]}\end{array}$ & $\begin{array}{l}\text { Height } \\
\text { [mAU] }\end{array}$ & $\begin{array}{c}\text { Area } \\
\text { so }\end{array}$ \\
\hline \multicolumn{6}{|c|}{$----|-------|----|-------|----------|----------|-------n$} \\
\hline 1 & $19.938 \mathrm{MM}$ & 0.4458 & 6933.33643 & 259.19455 & 51.8567 \\
\hline 2 & $23.518 \mathrm{MM}$ & 0.5200 & 6436.85059 & 206.31245 & 48.1433 \\
\hline ot & : & & $1.33702 \mathrm{e} 4$ & 465.50700 & \\
\hline
\end{tabular}

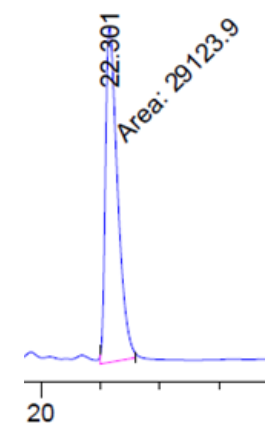

Signal 1: DAD1 A, Sig=254,4 Ref $=360,100$

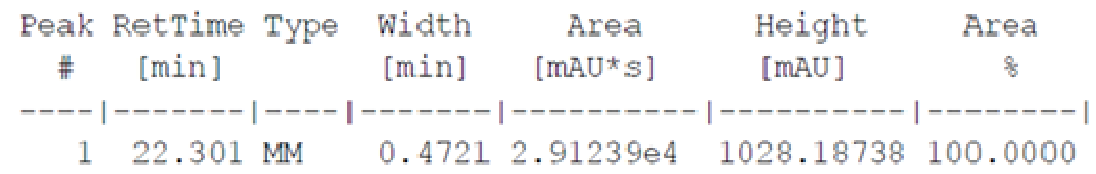

\section{Circular Dichroism data}

\begin{tabular}{c|cccccc} 
Band & $\left(\boldsymbol{R}_{\mathrm{p}}\right)-\mathbf{1 a}$ & $\left(\boldsymbol{R}_{\mathrm{p}}\right)-\mathbf{1 b}$ & $\left(\boldsymbol{R}_{\mathrm{p}}\right)-\mathbf{1 c}$ & $\left(\boldsymbol{R}_{\mathrm{p}}\right)-\mathbf{1 d}$ & $\left(\boldsymbol{R}_{\mathrm{p}}\right)-\mathbf{1 2}$ & $\left(\boldsymbol{R}_{\mathrm{p}}\right)-\mathbf{5}$ \\
& $\lambda(\Delta \varepsilon)$ & $\lambda(\Delta \varepsilon)$ & $\lambda(\Delta \varepsilon)$ & $\lambda(\Delta \varepsilon)$ & $\lambda(\Delta \varepsilon)$ & $\lambda(\Delta \varepsilon)$ \\
\hline \multirow{2}{*}{$\mathbf{2}$} & $255 \mathrm{~nm}$ & $252 \mathrm{~nm}$ & $275 \mathrm{~nm}$ & $256 \mathrm{~nm}$ & $275 \mathrm{~nm}$ & $315 \mathrm{~nm}$ \\
& $(+6.4)$ & $(+16)$ & $(+15)$ & $(+20)$ & $(+31)$ & $(+165)$ \\
\multirow{2}{*}{$\mathbf{3}$} & $288 \mathrm{~nm}$ & $291 \mathrm{~nm}$ & $329 \mathrm{~nm}$ & $280 \mathrm{~nm}$ & $304 \mathrm{~nm}$ & $350 \mathrm{~nm}$ \\
& $(+4.9)$ & $(+9.7)$ & $(+4.6)$ & $(+12)$ & $(+22)$ & $(+45)$ \\
& $323 \mathrm{~nm}$ & $320 \mathrm{~nm}$ & $367 \mathrm{~nm}$ & $321 \mathrm{~nm}$ & $409 \mathrm{~nm}$ & $438 \mathrm{~nm}$ \\
& $(+2.6)$ & $(+6.3)$ & $(+3.7)$ & $(+7.1)$ & $(+1.6)$ & $(+13.7)$ \\
& $528 \mathrm{~nm}$ & $520 \mathrm{~nm}$ & $533 \mathrm{~nm}$ & $514 \mathrm{~nm}$ & $533 \mathrm{~nm}$ & $511 \mathrm{~nm}$ \\
& $(+0.9)$ & $(+2.9)$ & $(+2.0)$ & $(+2.1)$ & $(+2.0)$ & $(+13.9)$ \\
\cline { 2 - 7 } & & & & & &
\end{tabular}


UV-vis spectra for $\left(R_{\mathrm{P}}\right)-1 a,\left(R_{\mathrm{P}}\right)-12$ and $\left(R_{\mathrm{P}}\right)-5$

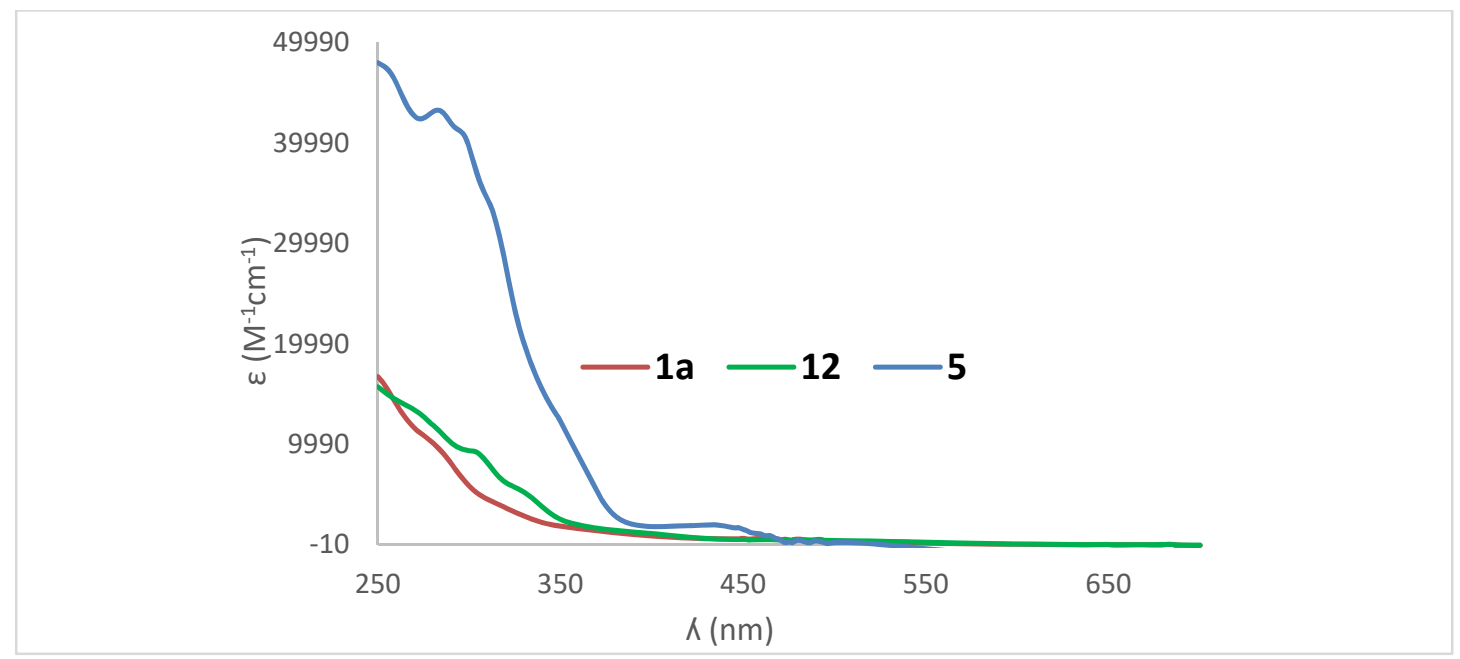

\section{UV-vis data}

\begin{tabular}{|c|ccc|}
\hline Band & $\left(\boldsymbol{R}_{\mathrm{P}}\right)-\mathbf{1 a} \varepsilon\left(\mathrm{cm}^{-1} \mathrm{M}^{-1}\right)$ & $\left(\boldsymbol{R}_{\mathrm{P}}\right)-\mathbf{1 2} \varepsilon\left(\mathrm{cm}^{-1} \mathrm{M}^{-1}\right)$ & $\left(\boldsymbol{R}_{\mathrm{P}}\right)-\mathbf{5} \varepsilon\left(\mathrm{cm}^{-1} \mathrm{M}^{-1}\right)$ \\
\hline $\mathbf{1}$ & $253 \mathrm{~nm}(16073)$, & $254 \mathrm{~nm}(15138)$, & $254 \mathrm{~nm}(47557)$, \\
$\mathbf{2}$ & $284 \mathrm{~nm}(9460)$ & $307 \mathrm{~nm}(8800)$ & $292 \mathrm{~nm}(41566)$ \\
\hline
\end{tabular}




\section{$\underline{\text { X-Ray Crystallography for }\left(\boldsymbol{R}_{\mathrm{p}}\right)-1 \mathrm{C} \text { (CCDC-1469768) }}$}

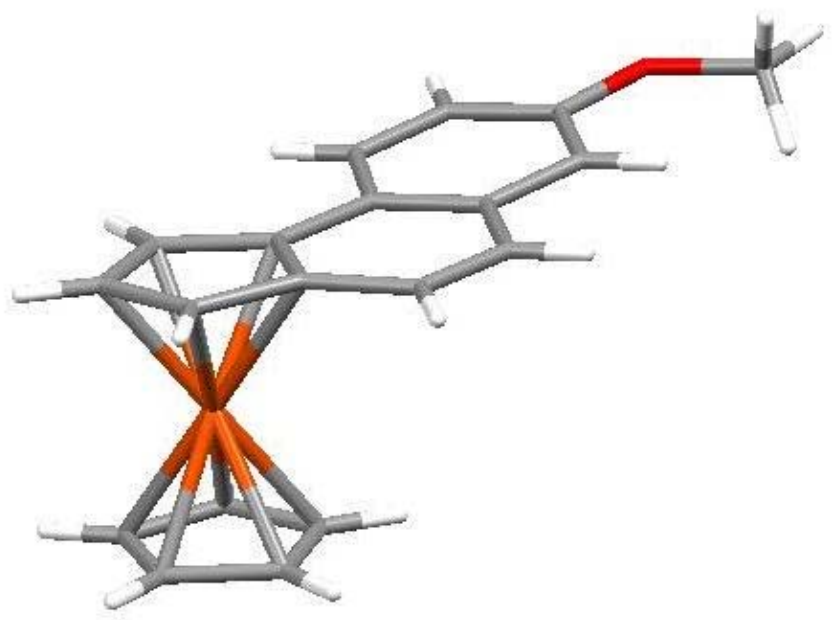

Table. Crystal data and structure refinement for $\left(R_{\mathrm{p}}\right)-1 \mathrm{c}$.

\begin{tabular}{|c|c|c|}
\hline Identification code & $\left(R_{\mathrm{p}}\right)-1 \mathrm{c}$ & \\
\hline Chemical formula & $\mathrm{C}_{19} \mathrm{H}_{16} \mathrm{FeO}$ & \\
\hline Formula weight & 316.17 & \\
\hline Temperature & $200(2) \mathrm{K}$ & \\
\hline Wavelength & $0.71073 \AA$ & \\
\hline Crystal size & $0.02 \times 0.14 \times 0.18 \mathrm{~mm}$ & \\
\hline Crystal habit & clear light red plate & \\
\hline Crystal system & tetragonal & \\
\hline Space group & P 41212 & \\
\hline \multirow[t]{3}{*}{ Unit cell dimensions } & $a=7.8273(13) \AA$ & $\alpha=90^{\circ}$ \\
\hline & $b=7.8273(13) \AA$ & $\beta=90^{\circ}$ \\
\hline & $c=47.227(9) \AA$ & $\gamma=90^{\circ}$ \\
\hline Volume & $2893.4(9) \AA^{3}$ & \\
\hline Z & 8 & \\
\hline Density (calculated) & $1.452 \mathrm{Mg} / \mathrm{cm}^{3}$ & \\
\hline Absorption coefficient & $1.037 \mathrm{~mm}^{-1}$ & \\
\hline$F(000)$ & 1312 & \\
\hline
\end{tabular}




\begin{tabular}{|c|c|c|}
\hline $\begin{array}{l}\text { Theta range for data } \\
\text { collection }\end{array}$ & \multicolumn{2}{|c|}{1.72 to $25.45^{\circ}$} \\
\hline Index ranges & \multicolumn{2}{|c|}{$-7<=\mathrm{h}<=7,-9<=\mathrm{k}<=8,-48<=1<=57$} \\
\hline Reflections collected & \multicolumn{2}{|l|}{10547} \\
\hline Independent reflections & \multicolumn{2}{|c|}{$2679[R($ int $)=0.1009]$} \\
\hline $\begin{array}{l}\text { Coverage of independent } \\
\text { reflections }\end{array}$ & \multicolumn{2}{|l|}{$99.9 \%$} \\
\hline Absorption correction & \multicolumn{2}{|l|}{ multi-scan } \\
\hline Max. and min. transmission & \multicolumn{2}{|c|}{0.9796 and 0.8353} \\
\hline Structure solution technique & \multicolumn{2}{|c|}{ direct methods } \\
\hline Structure solution program & \multicolumn{2}{|c|}{ SHELXS-97 (Sheldrick, 2008) } \\
\hline Refinement method & \multicolumn{2}{|c|}{ Full-matrix least-squares on $\mathrm{F}^{2}$} \\
\hline Refinement program & \multicolumn{2}{|c|}{ SHELXL-97 (Sheldrick, 2008) } \\
\hline Function minimized & \multicolumn{2}{|c|}{$\Sigma w\left(F_{o}^{2}-F_{c}^{2}\right)^{2}$} \\
\hline $\begin{array}{l}\text { Data / restraints / } \\
\text { parameters }\end{array}$ & \multicolumn{2}{|c|}{2679 / 0 / 191} \\
\hline Goodness-of-fit on $F^{2}$ & \multicolumn{2}{|l|}{1.031} \\
\hline$\Delta / \sigma_{\max }$ & \multicolumn{2}{|l|}{0.001} \\
\hline \multirow[t]{2}{*}{ Final $\mathbf{R}$ indices } & $\begin{array}{l}1829 \text { data; } \\
I>2 \sigma(I)\end{array}$ & $\begin{array}{l}\mathrm{R} 1=0.0751, \mathrm{wR} 2= \\
0.1647\end{array}$ \\
\hline & all data & $\begin{array}{l}\mathrm{R} 1=0.1172, \mathrm{wR} 2= \\
0.1839\end{array}$ \\
\hline Weighting scheme & \multicolumn{2}{|c|}{$\begin{array}{l}w=1 /\left[\sigma^{2}\left(F_{o}^{2}\right)+(0.1000 P)^{2}+0.0000 P\right] \\
\text { where } P=\left(F_{o}^{2}+2 F_{c}^{2}\right) / 3\end{array}$} \\
\hline $\begin{array}{l}\text { Absolute structure } \\
\text { parameter }\end{array}$ & \multicolumn{2}{|l|}{$-0.0(1)$} \\
\hline Largest diff. peak and hole & \multicolumn{2}{|c|}{0.335 and $-0.441 \mathrm{e}^{-3}$} \\
\hline R.M.S. deviation from mean & \multicolumn{2}{|l|}{$0.083 \mathrm{e}^{-3}$} \\
\hline
\end{tabular}




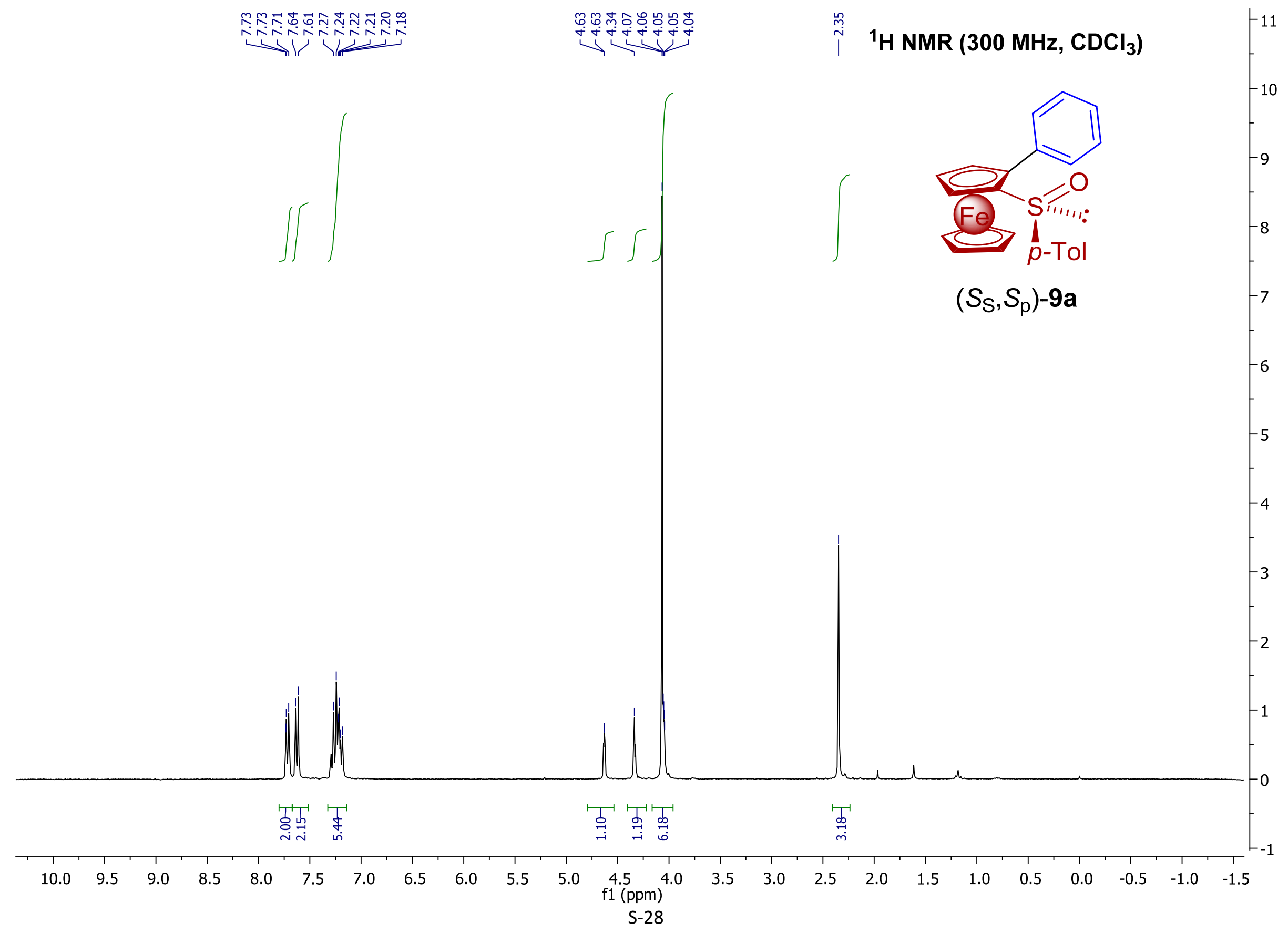




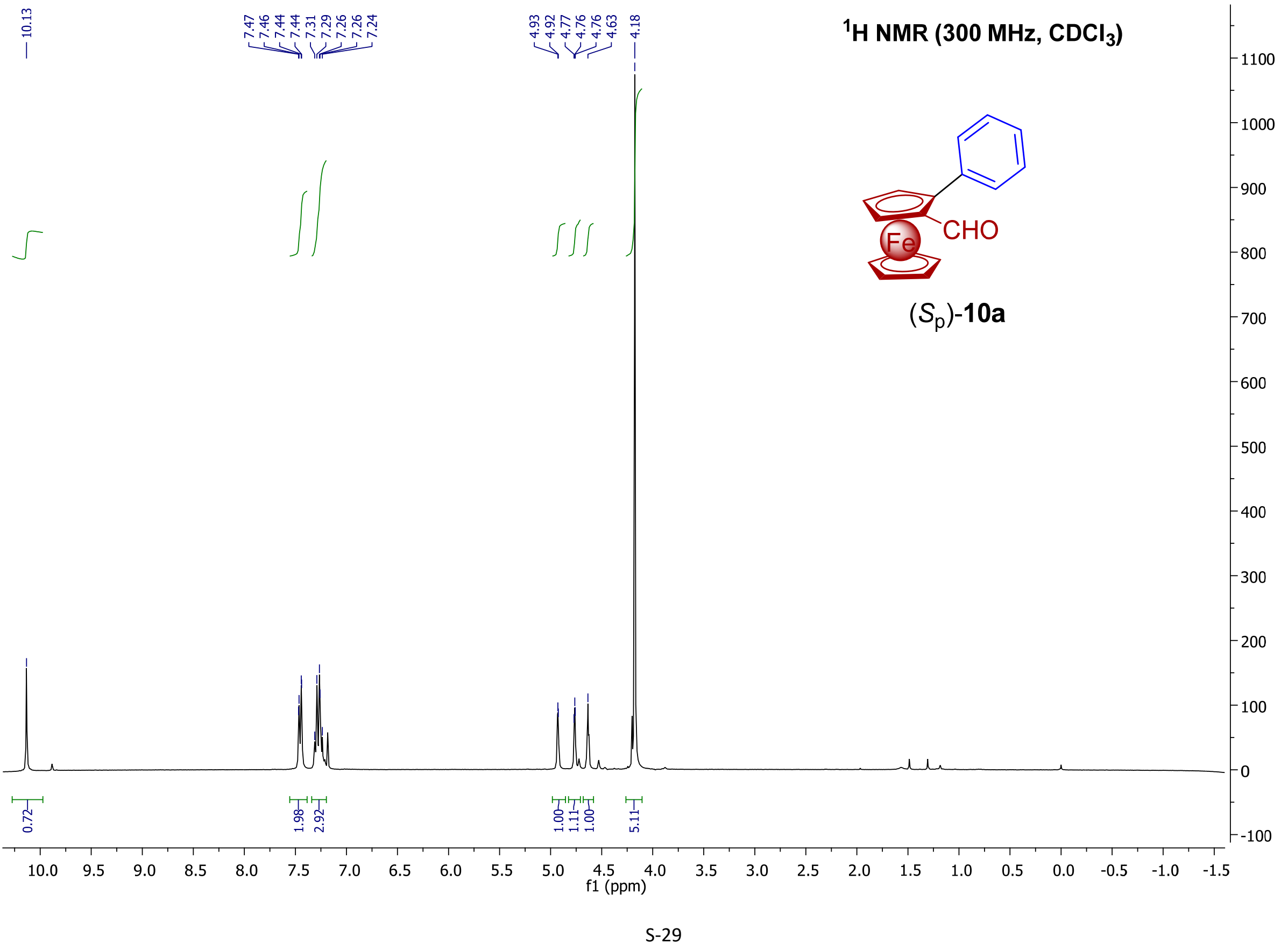




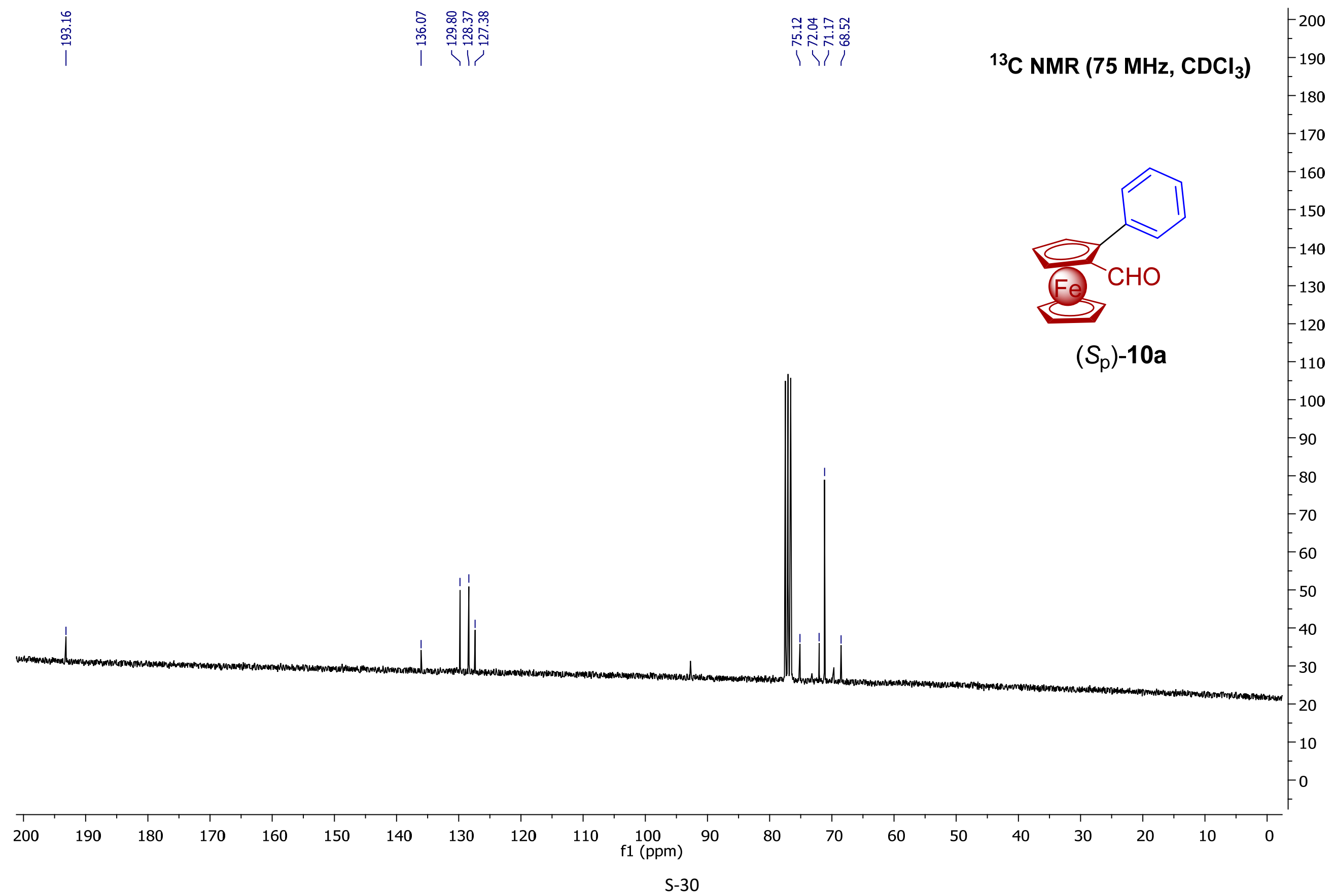




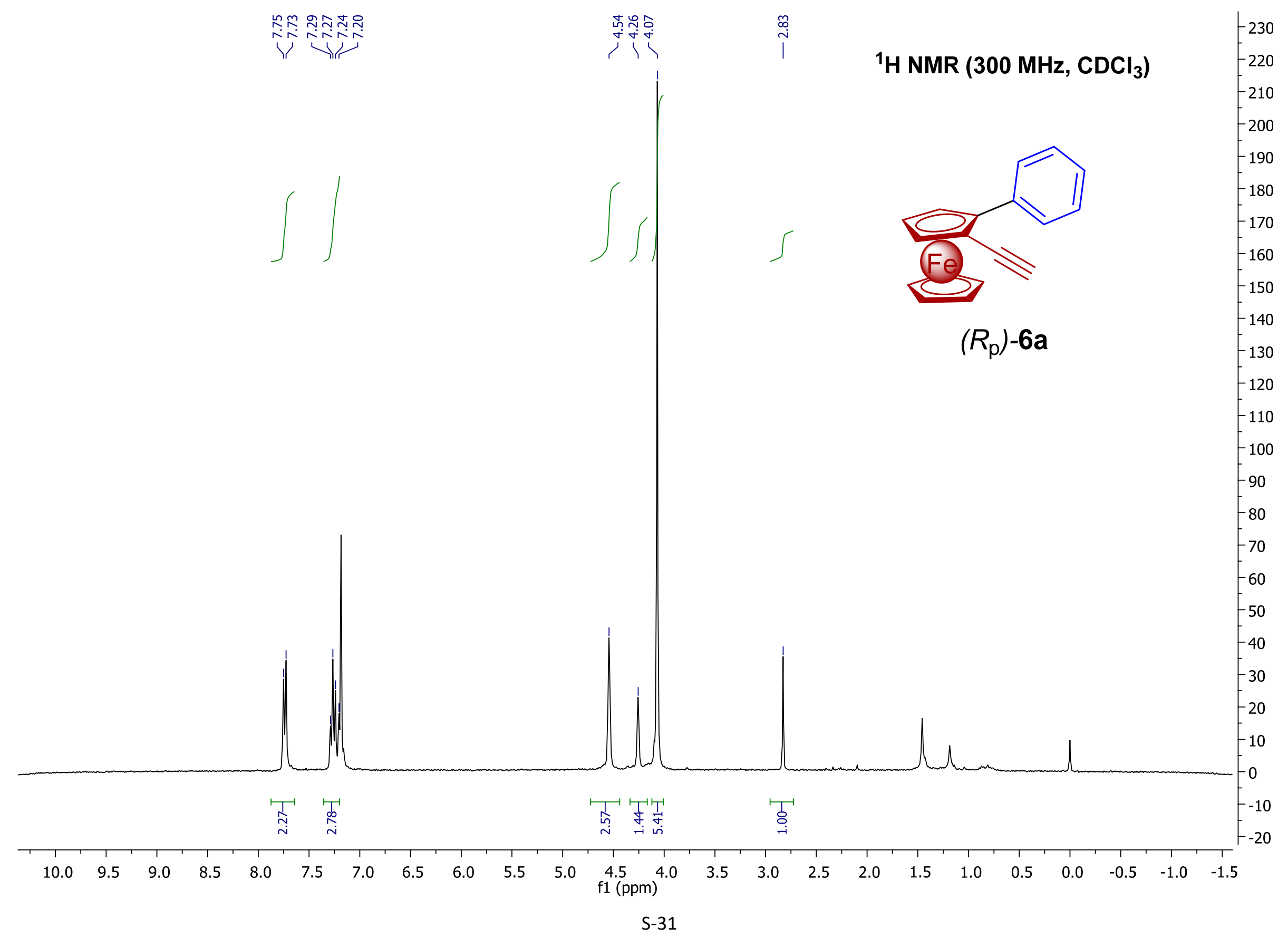




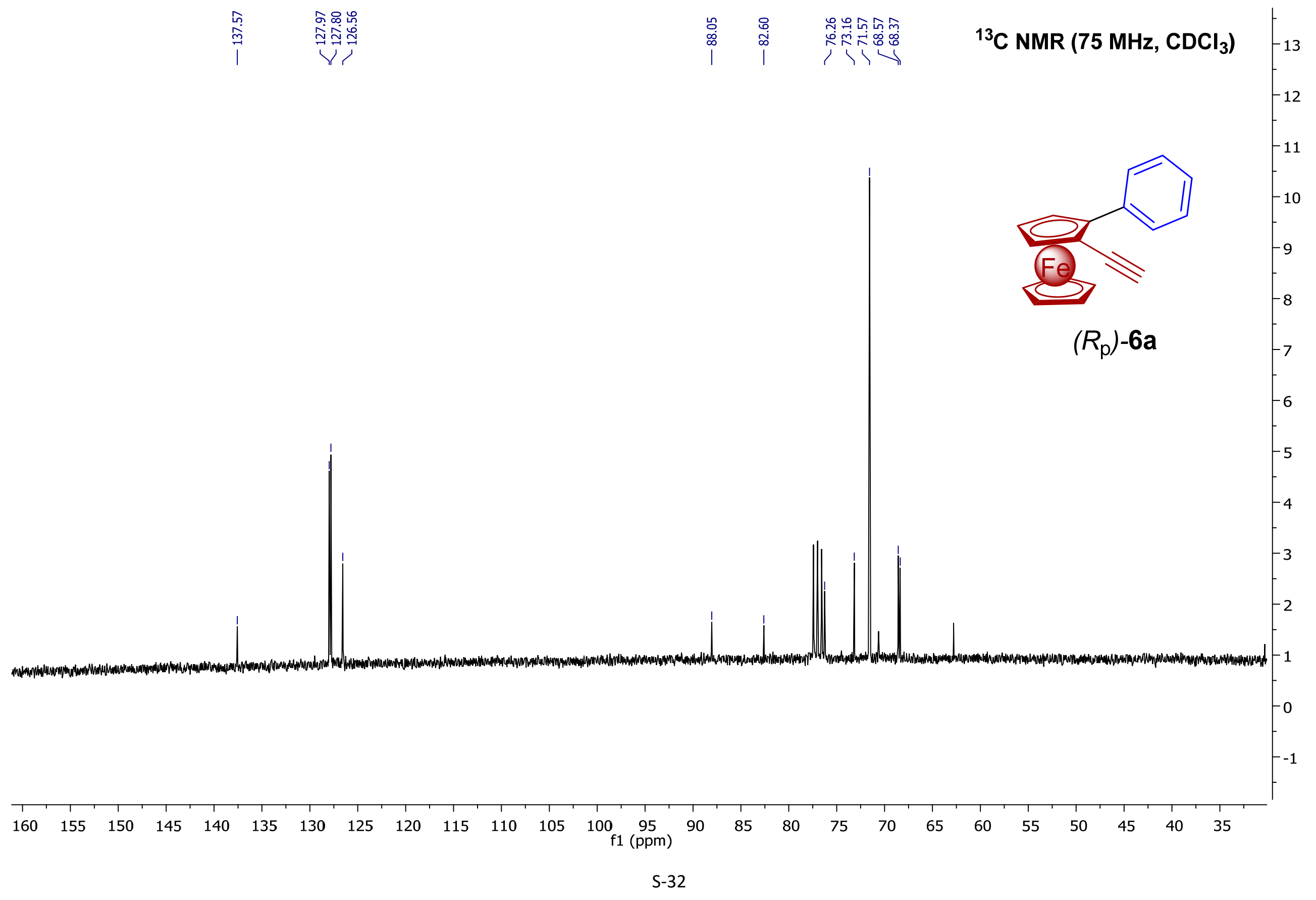




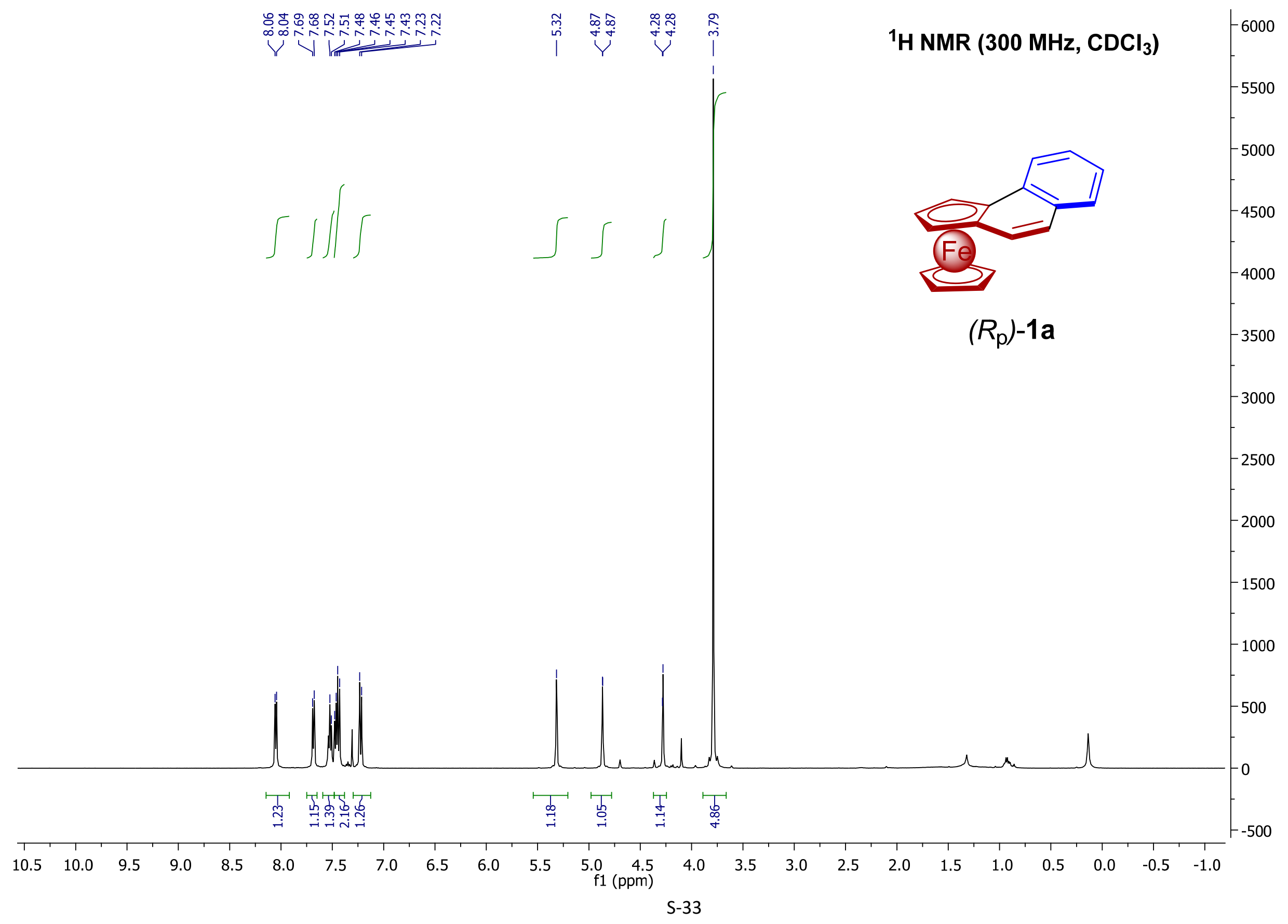




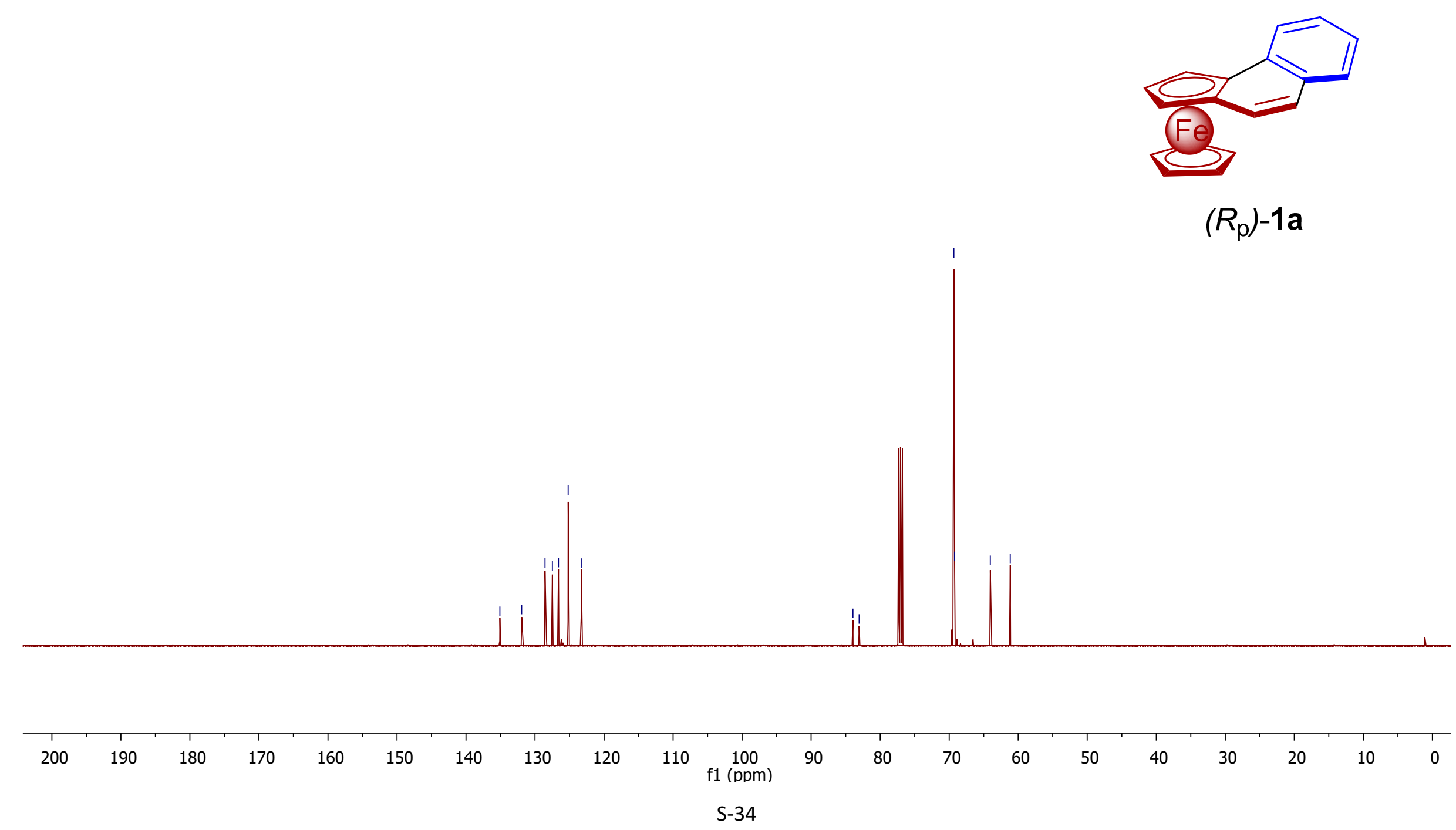




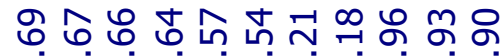
ベNヘヘヘNベ்

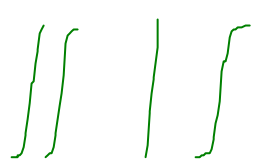

ஸุ

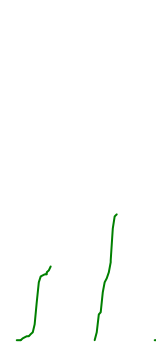

$\stackrel{\sim}{\sim}$

I

${ }^{1} \mathrm{H}$ NMR $\left(300 \mathrm{MHz}, \mathrm{CDCl}_{3}\right)$

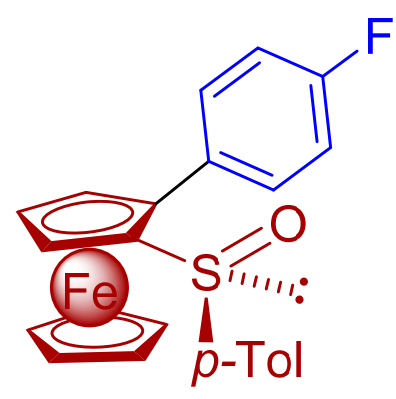

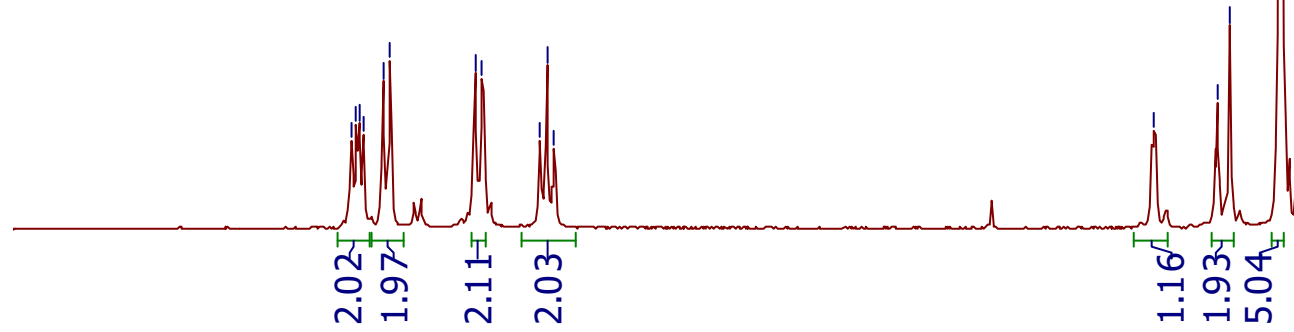

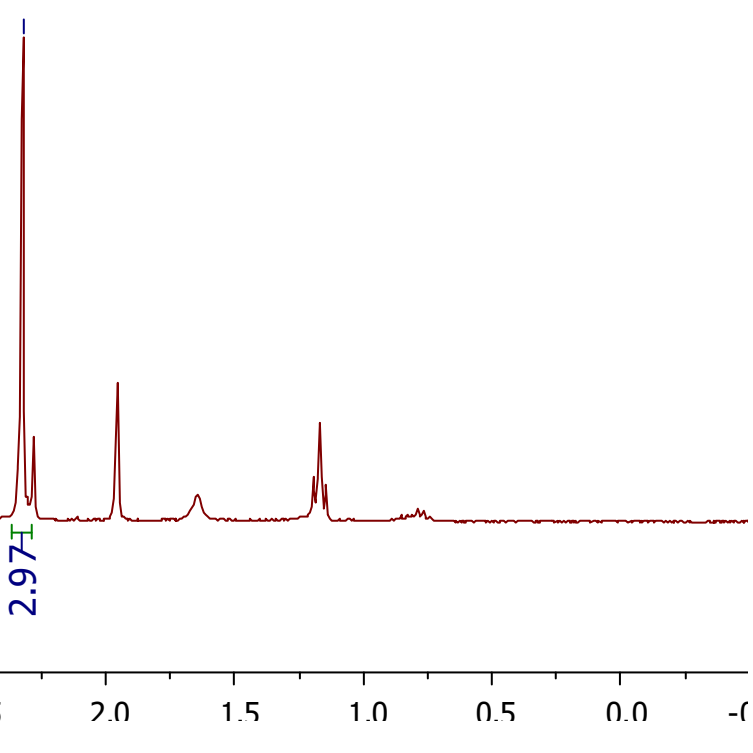




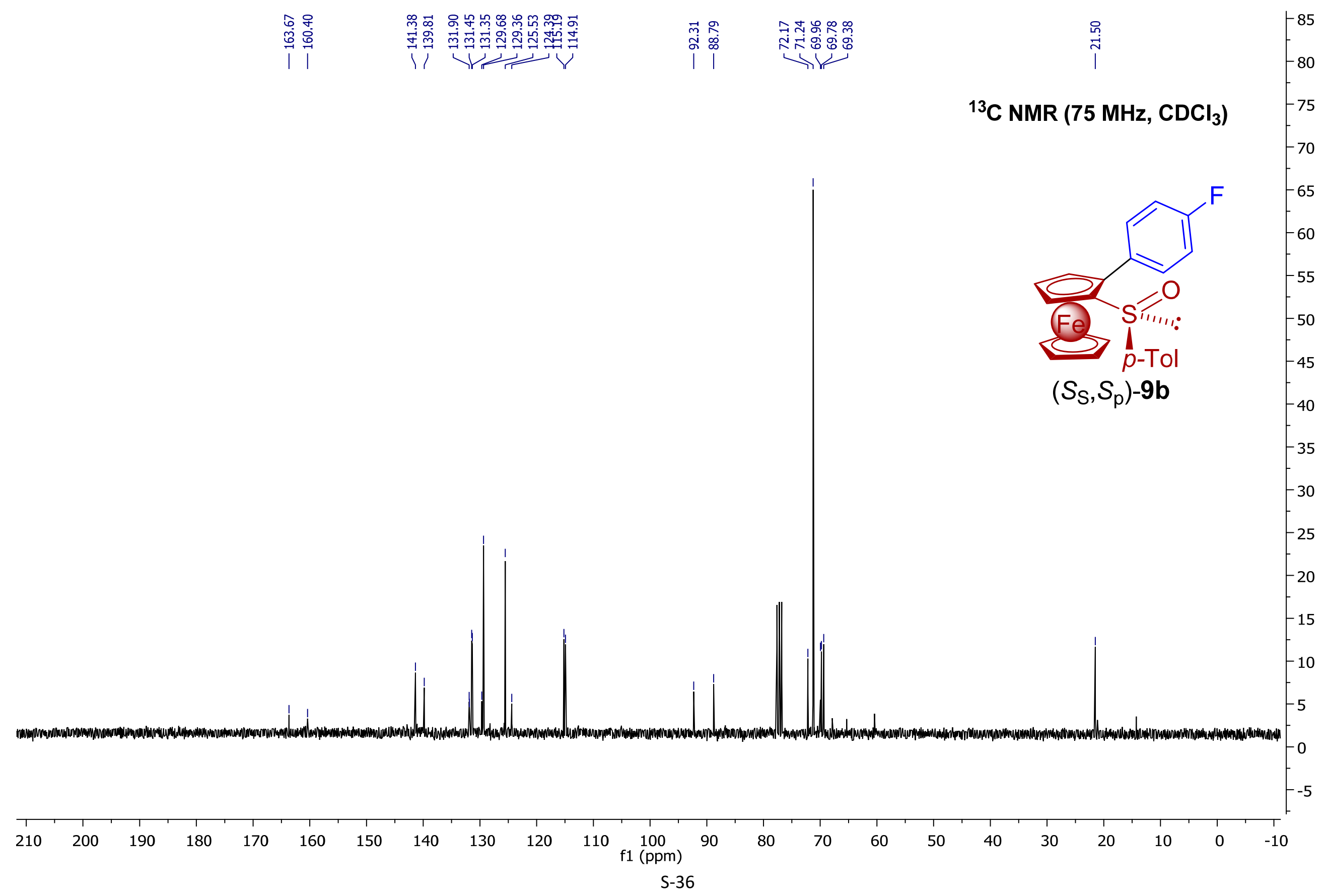


${ }^{19} \mathrm{~F}$ NMR $\left(282 \mathrm{MHz}, \mathrm{CDCl}_{3}\right)$

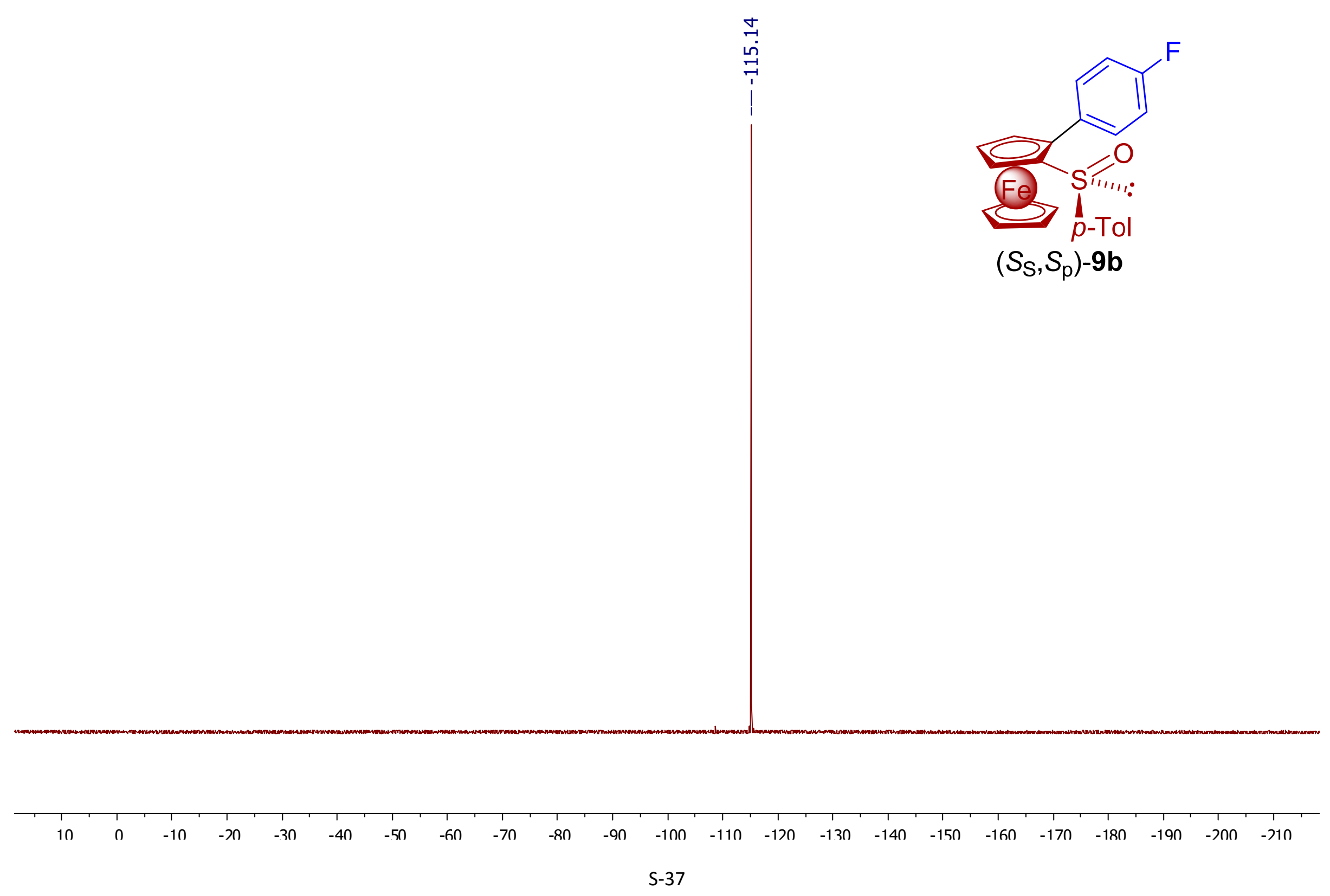




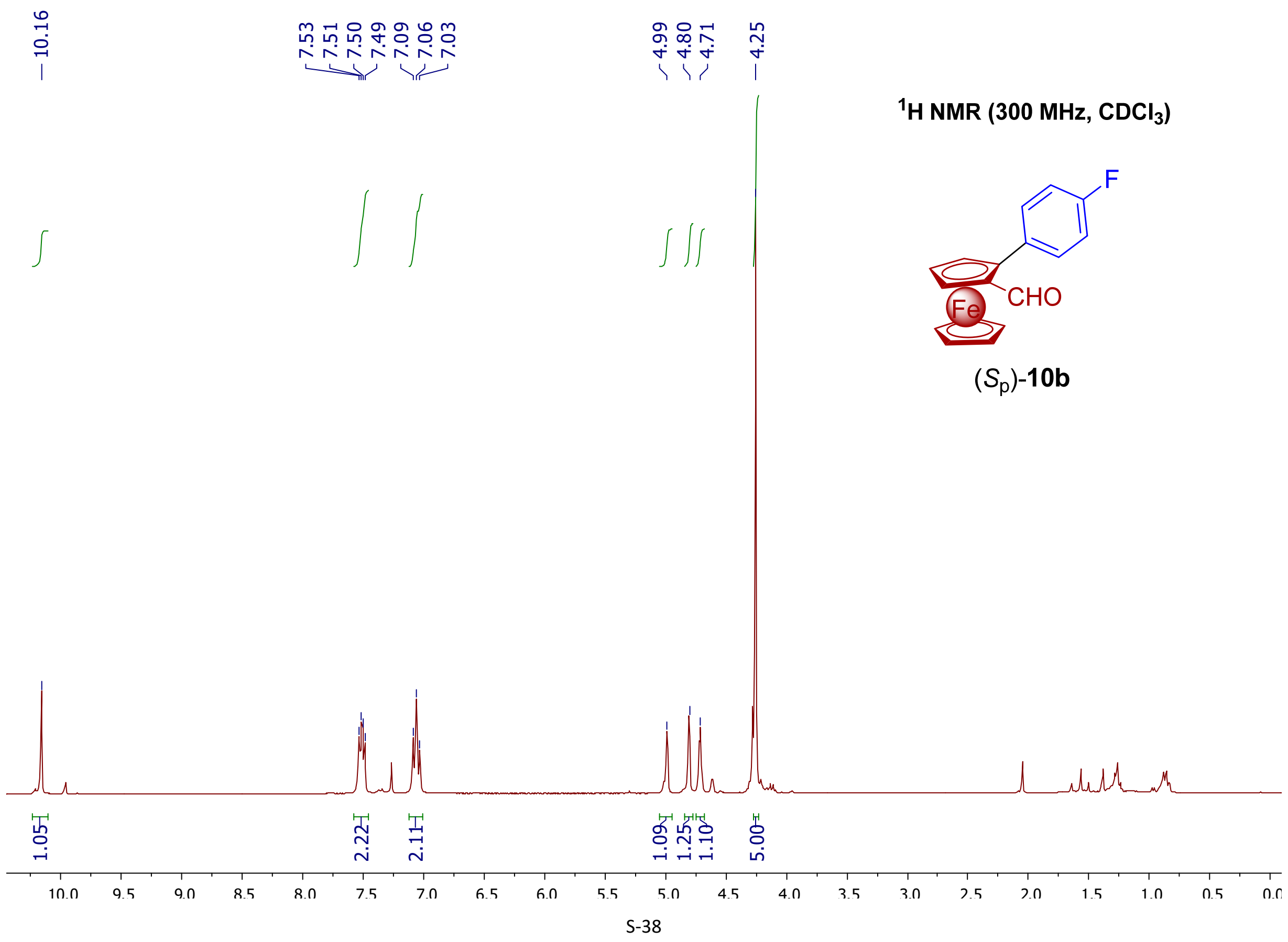




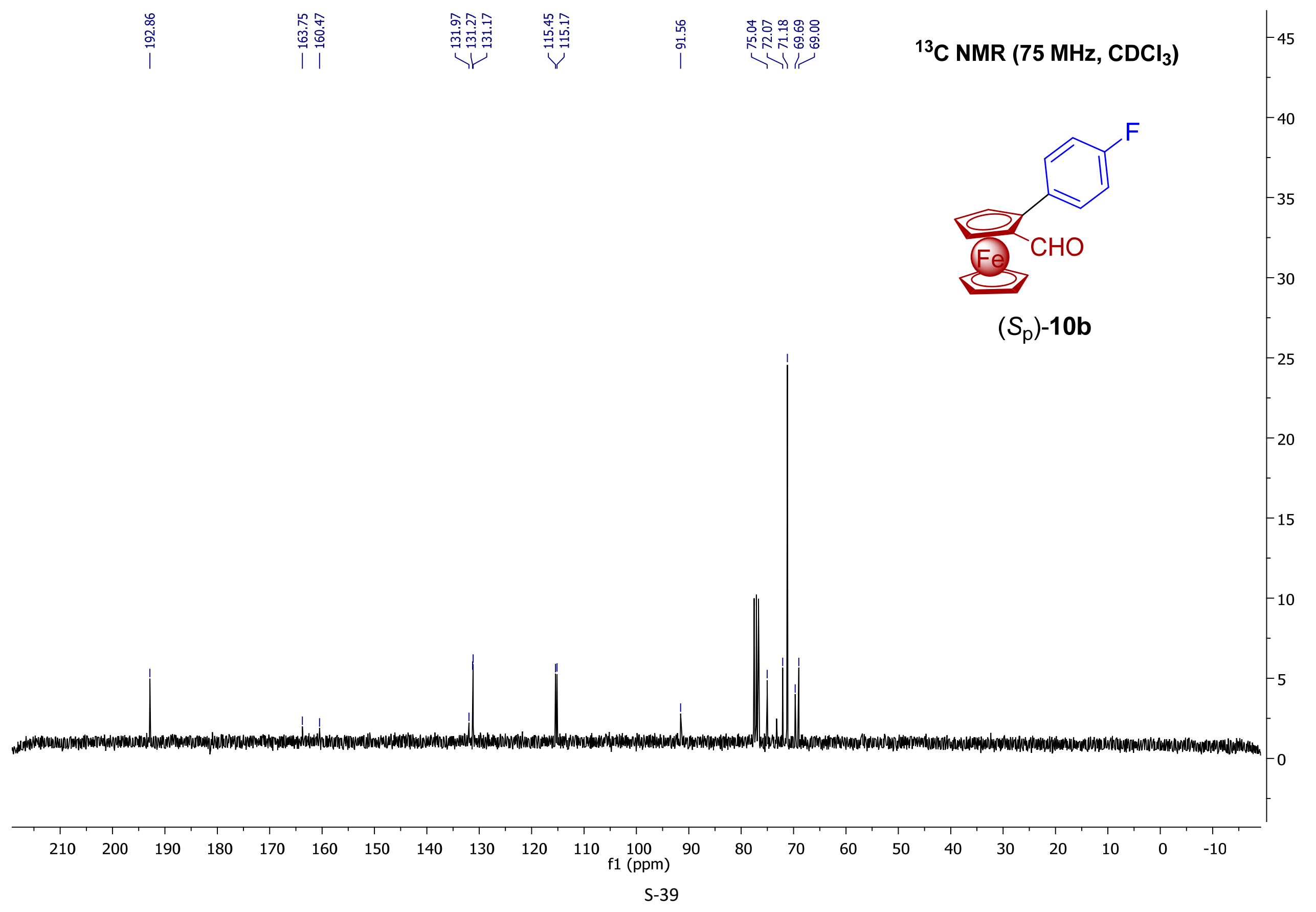


${ }^{19} \mathrm{~F}$ NMR $\left(282 \mathrm{MHz}, \mathrm{CDCl}_{3}\right)$

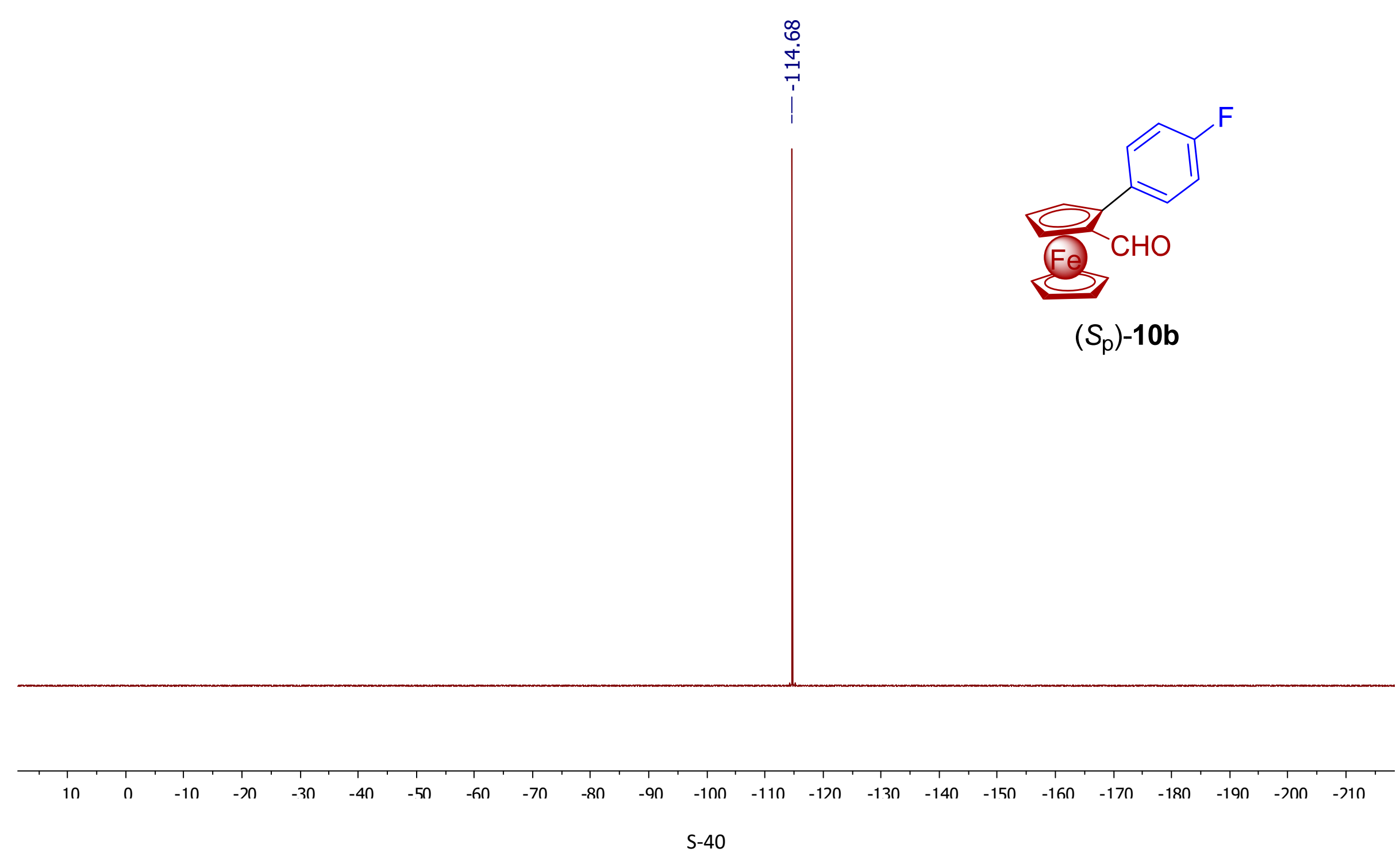




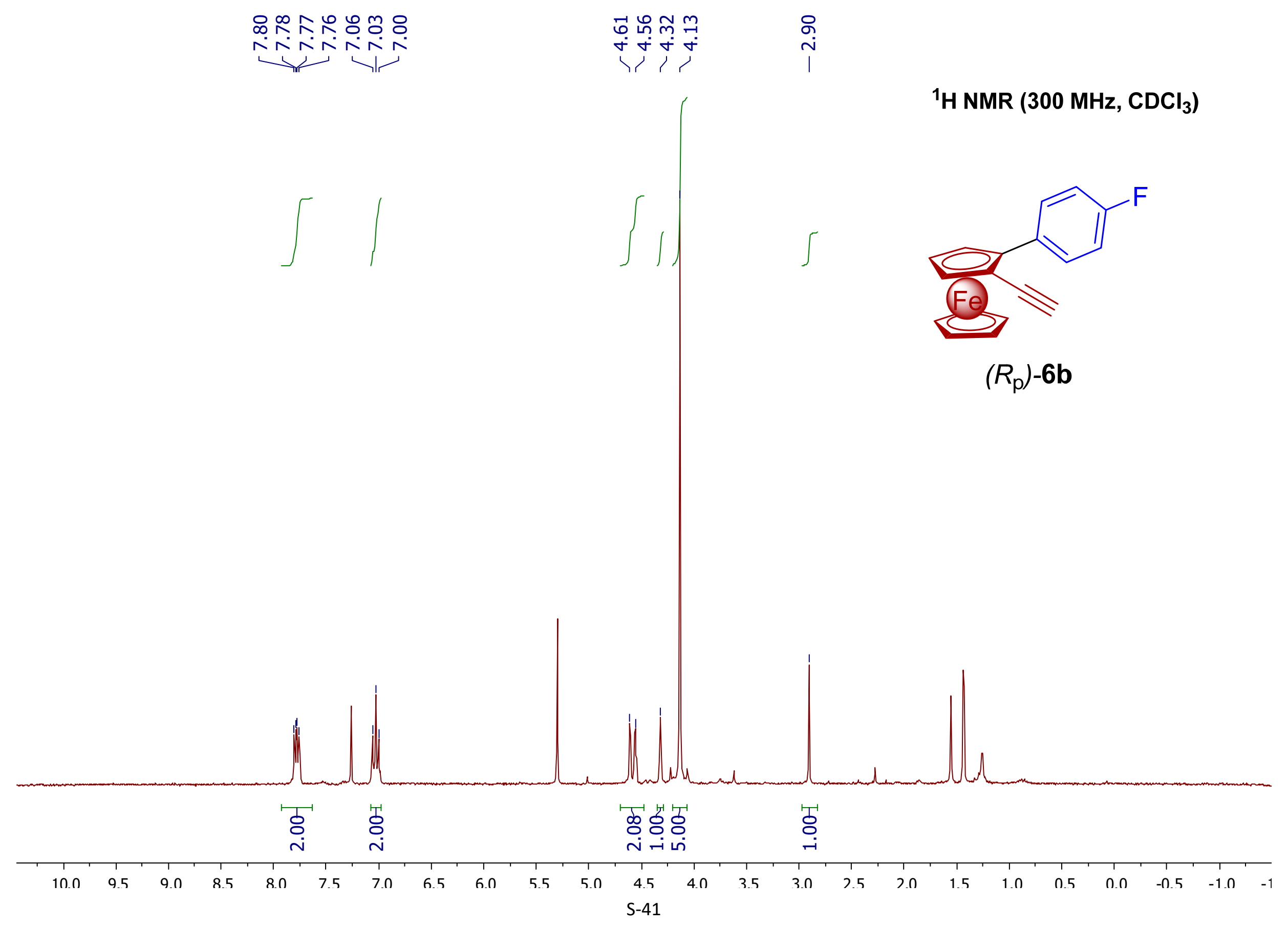




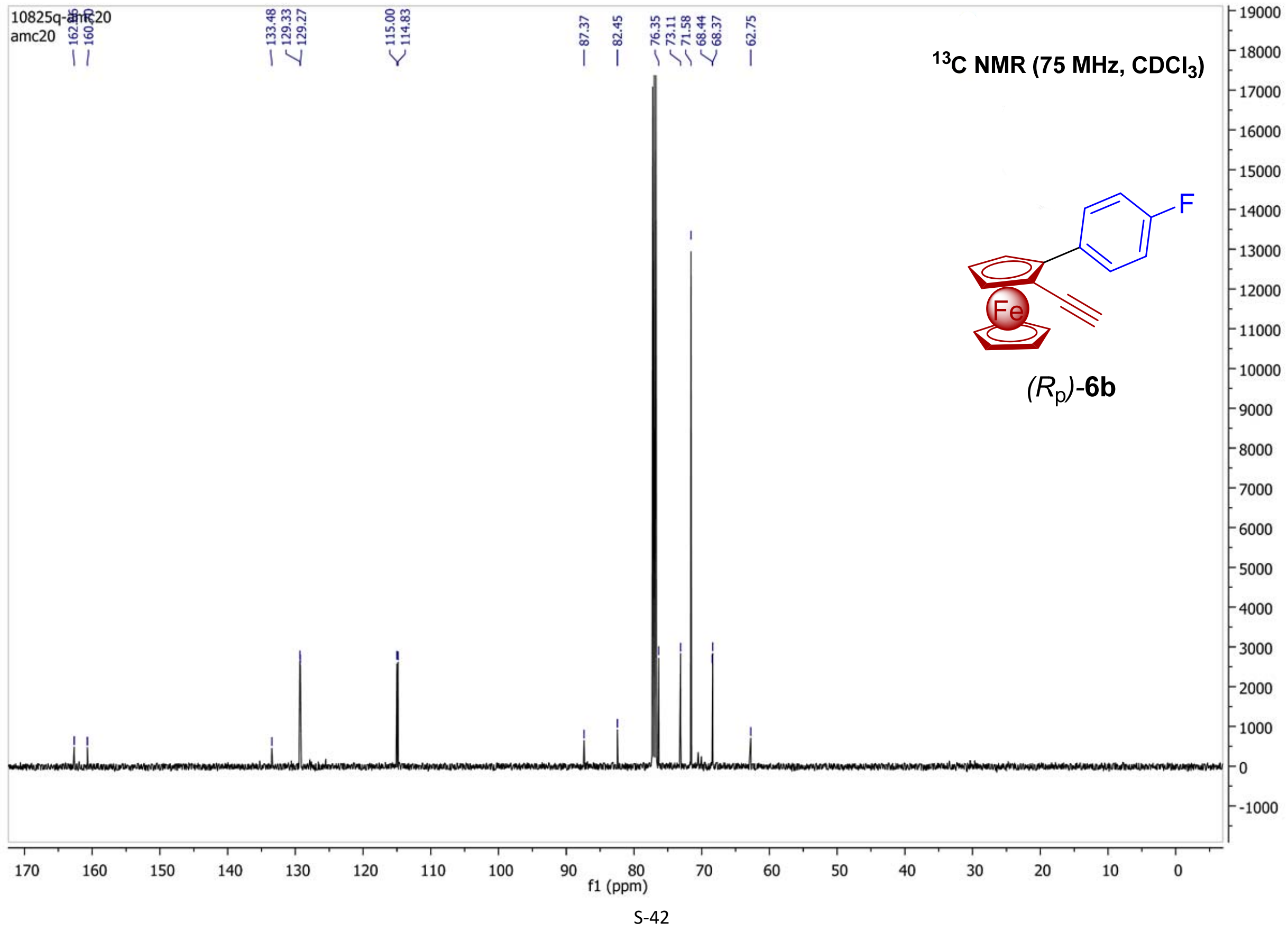




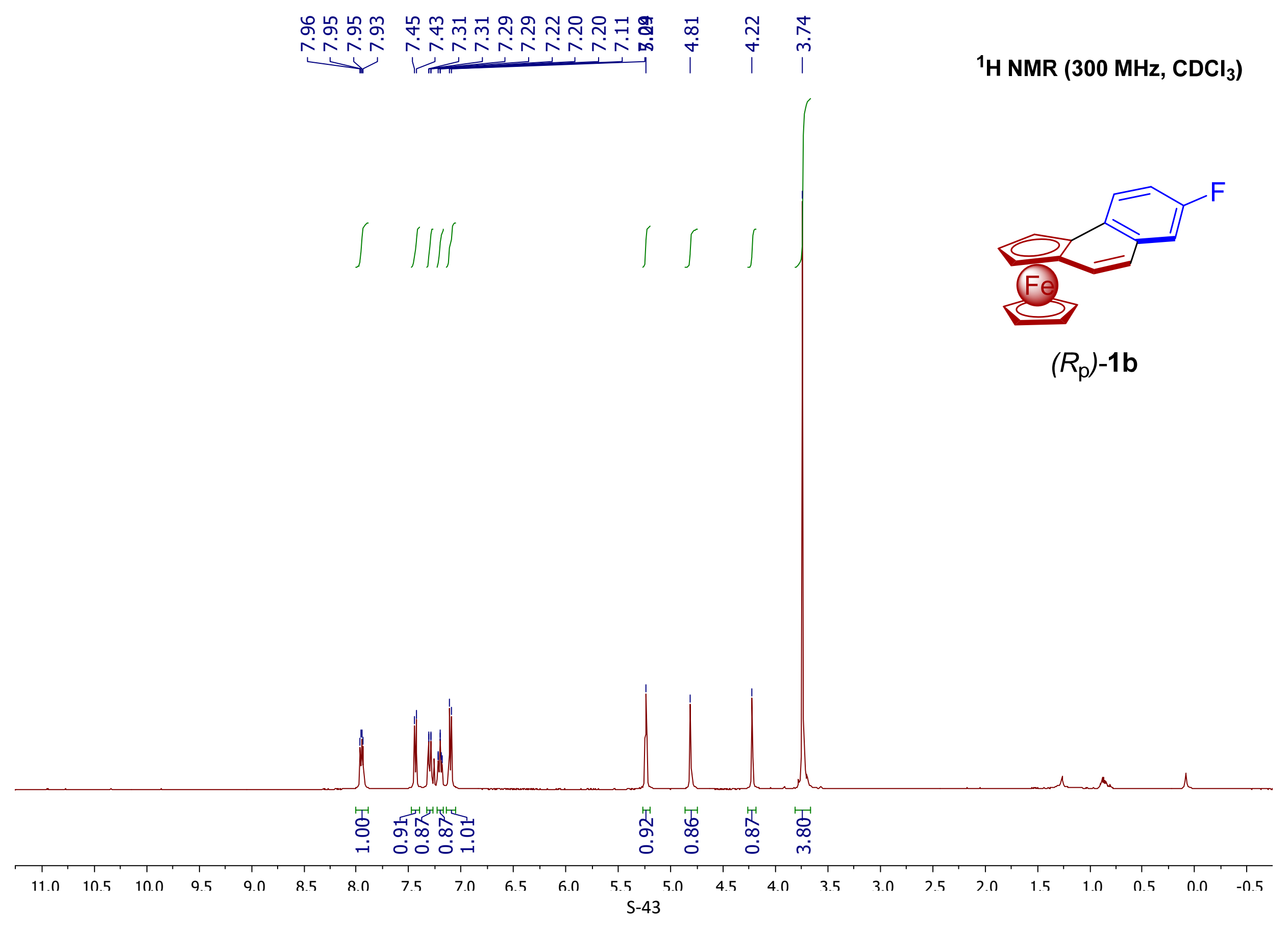




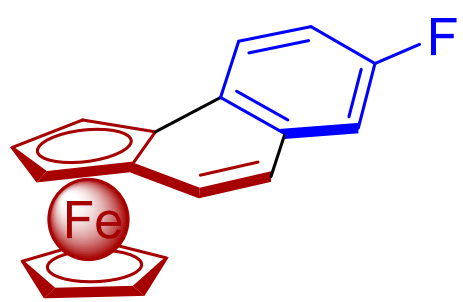

$$
\left(R_{\mathrm{p}}\right)-1 \mathrm{~b}
$$

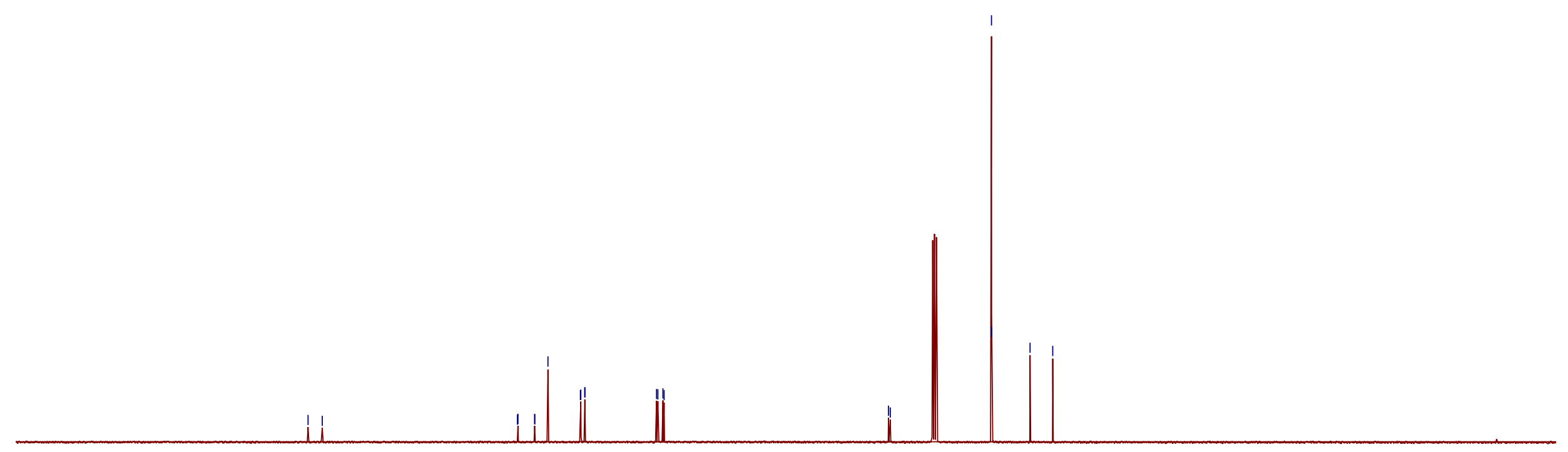

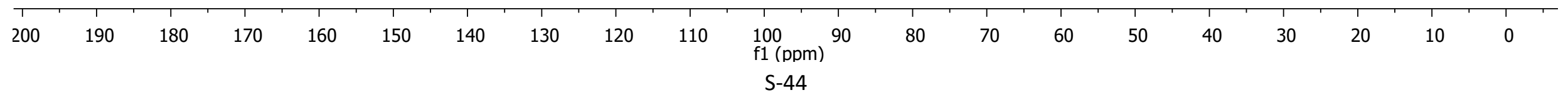




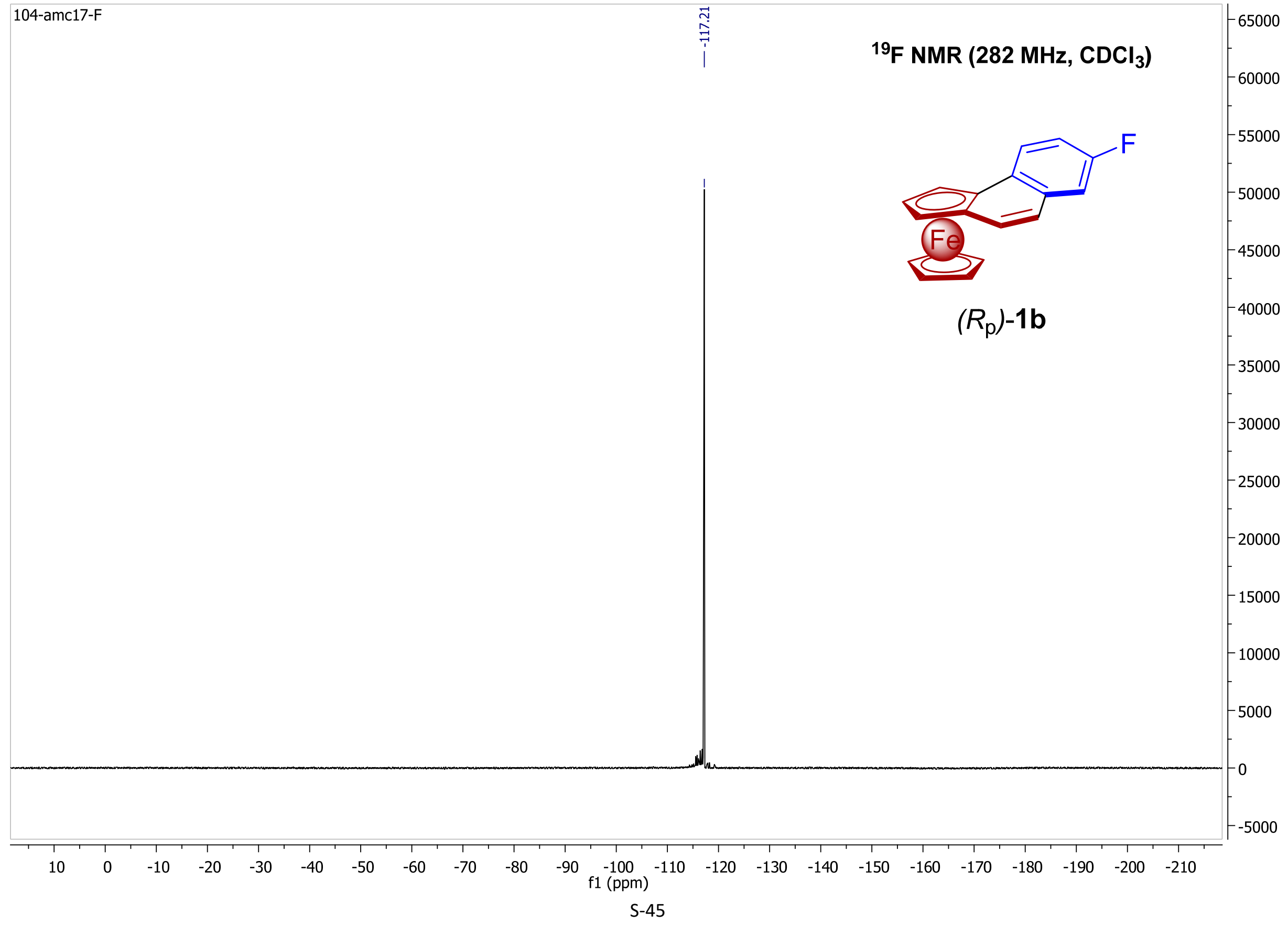




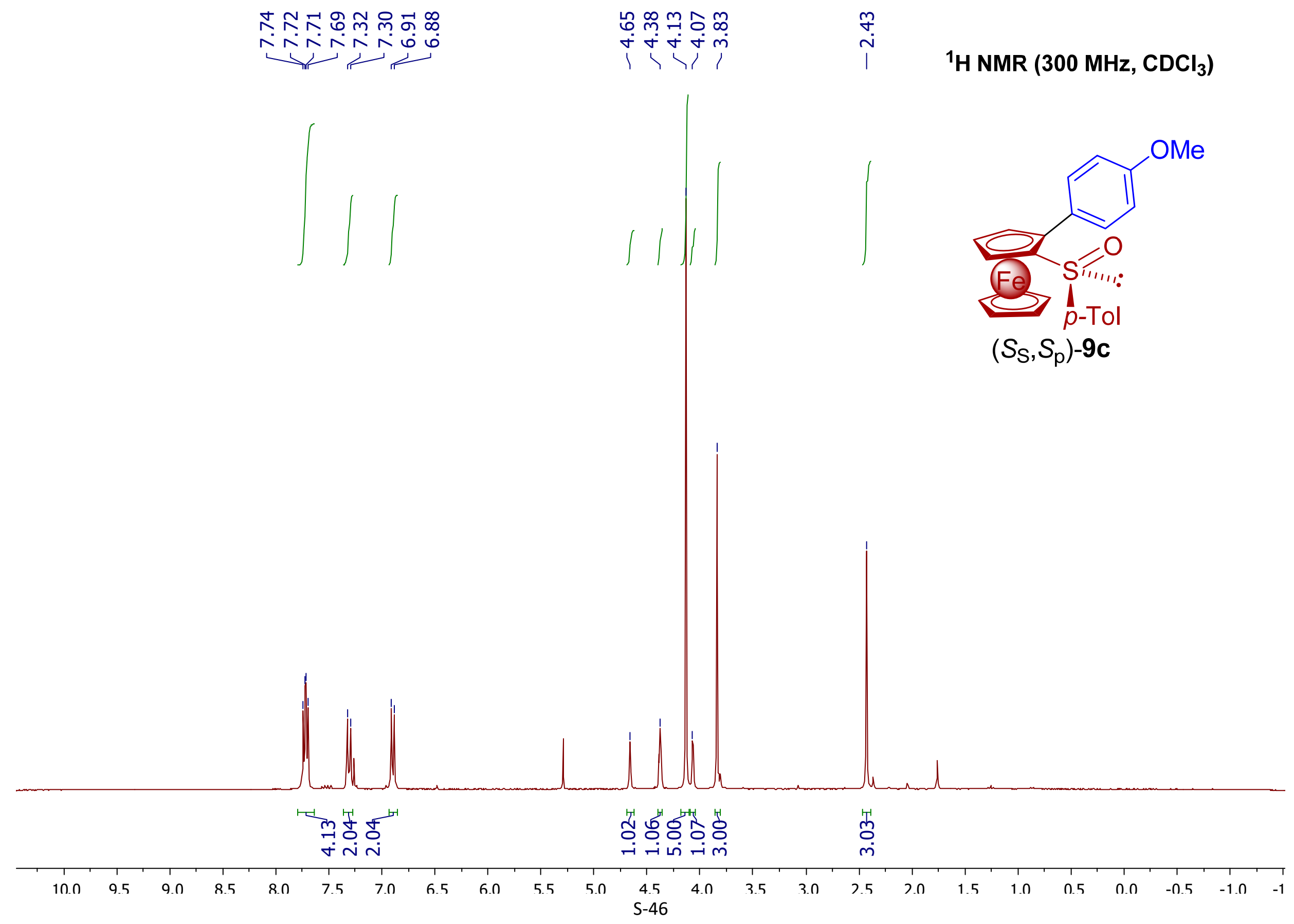




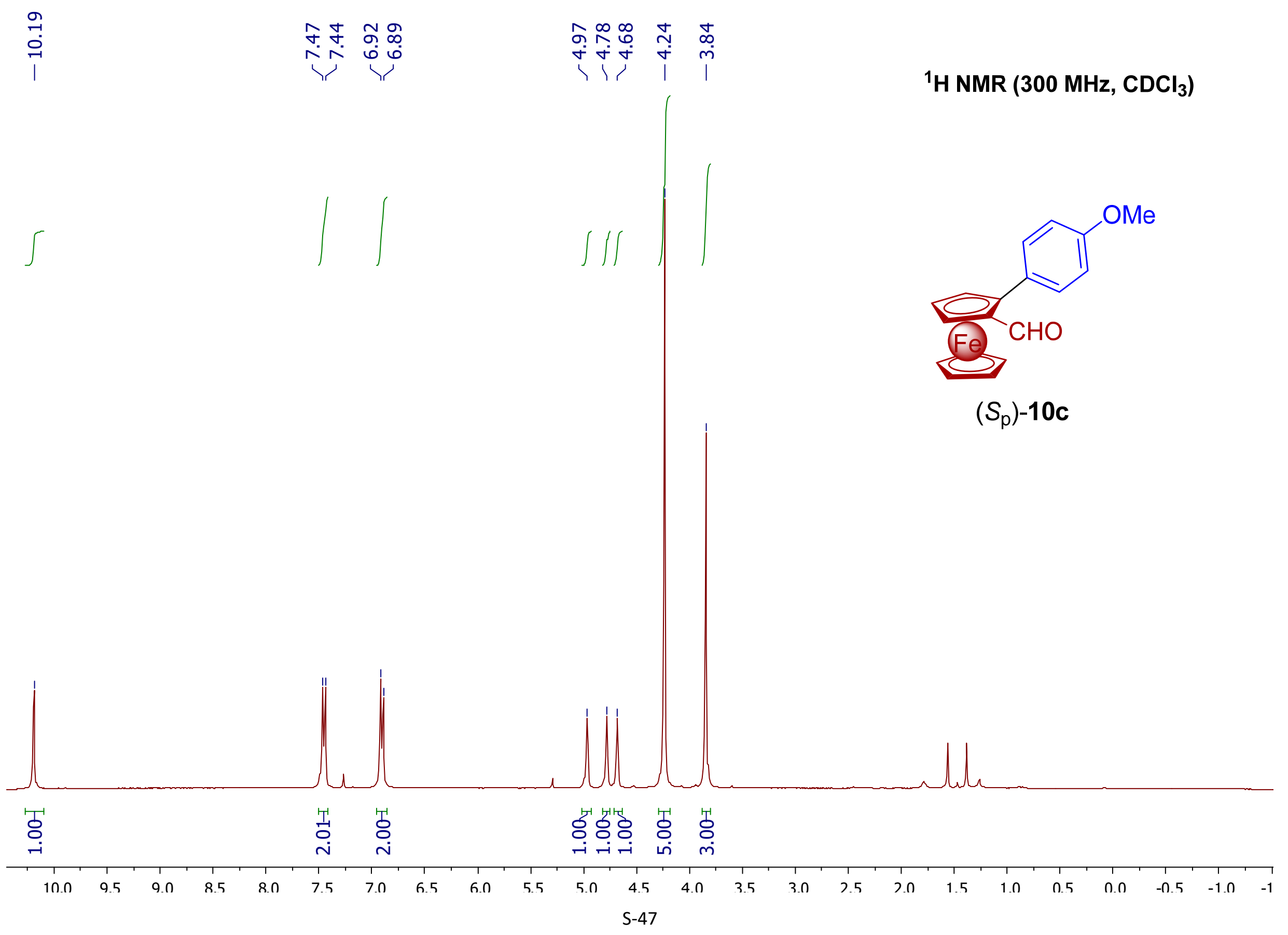




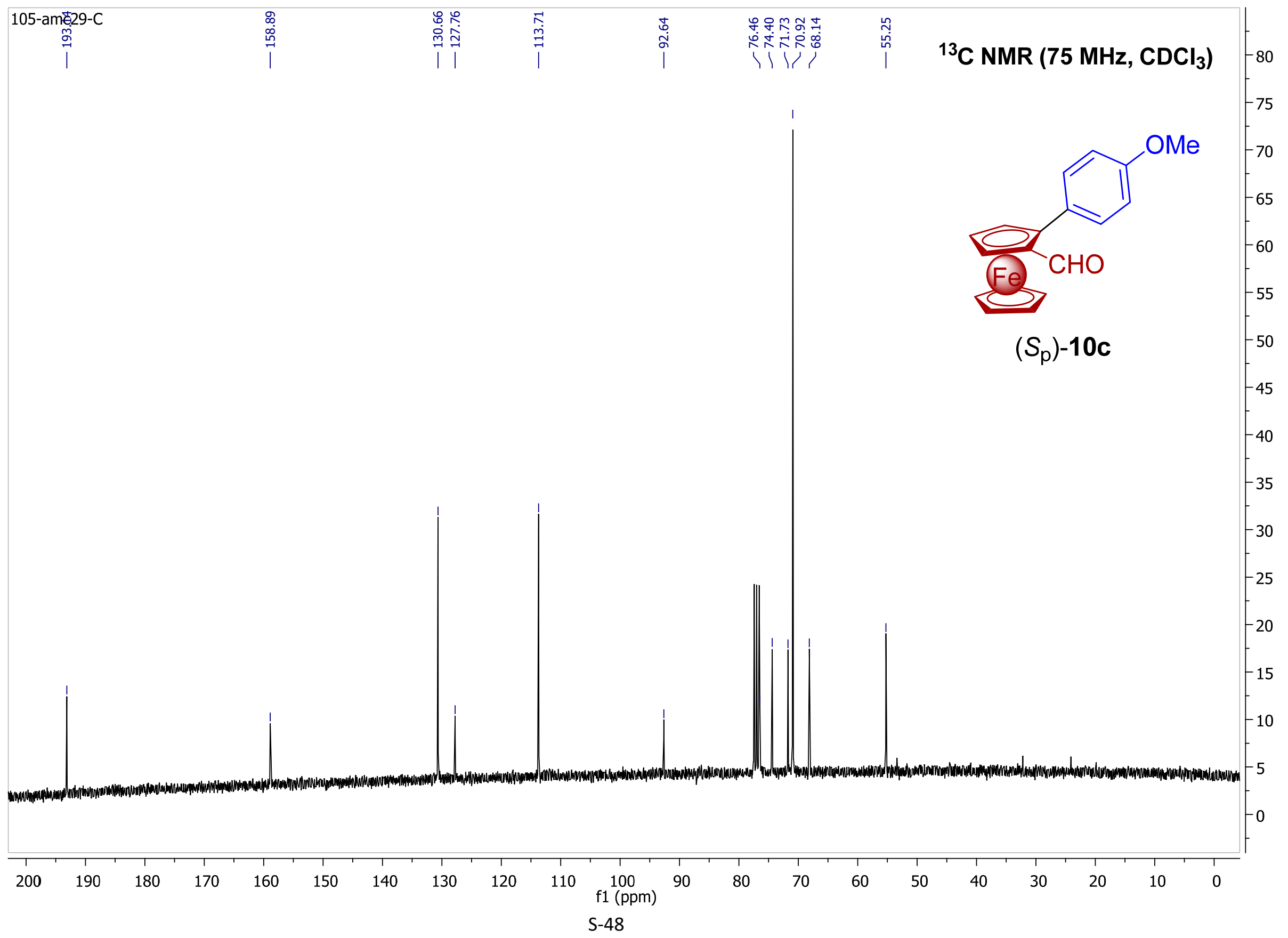




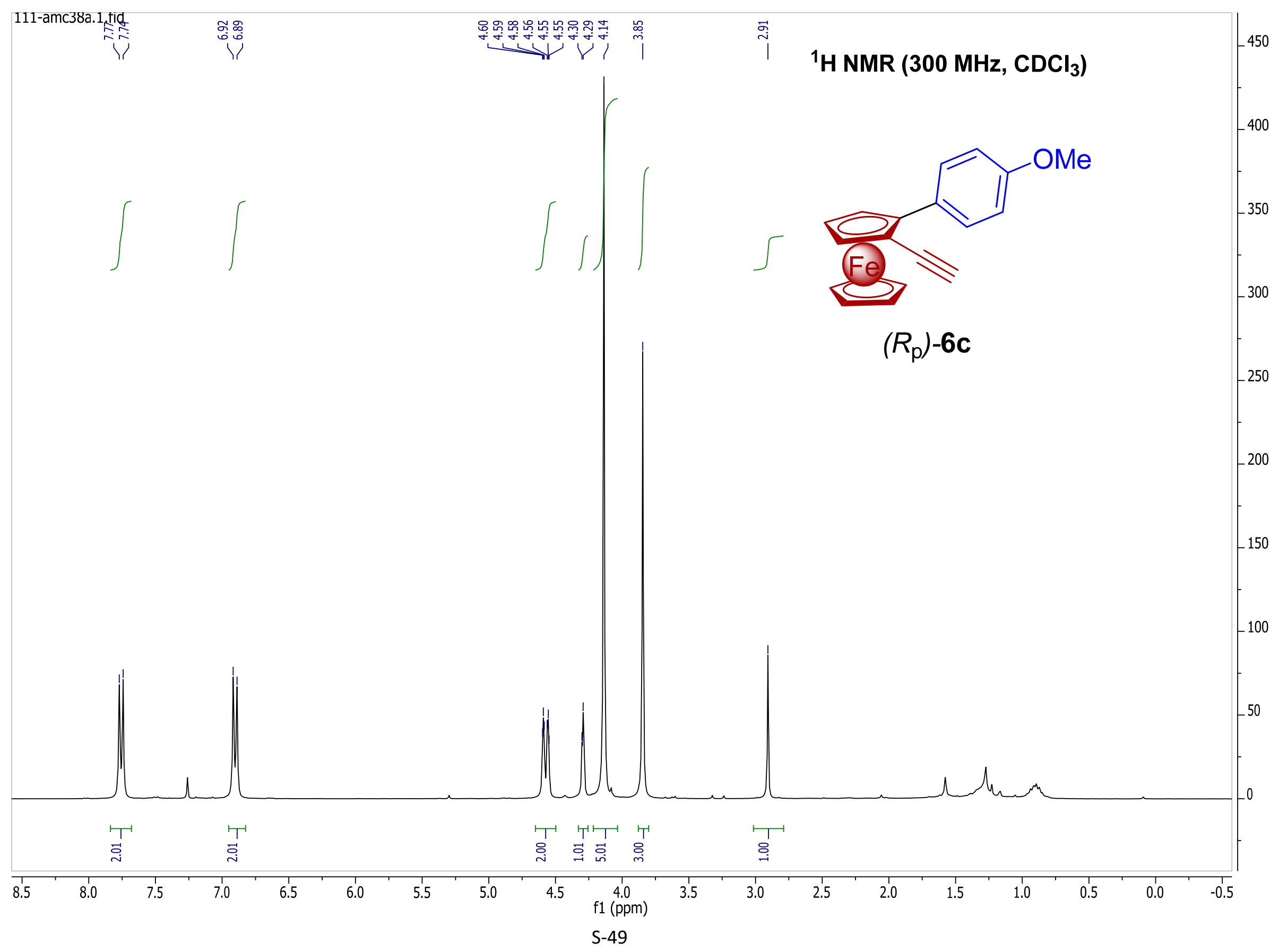




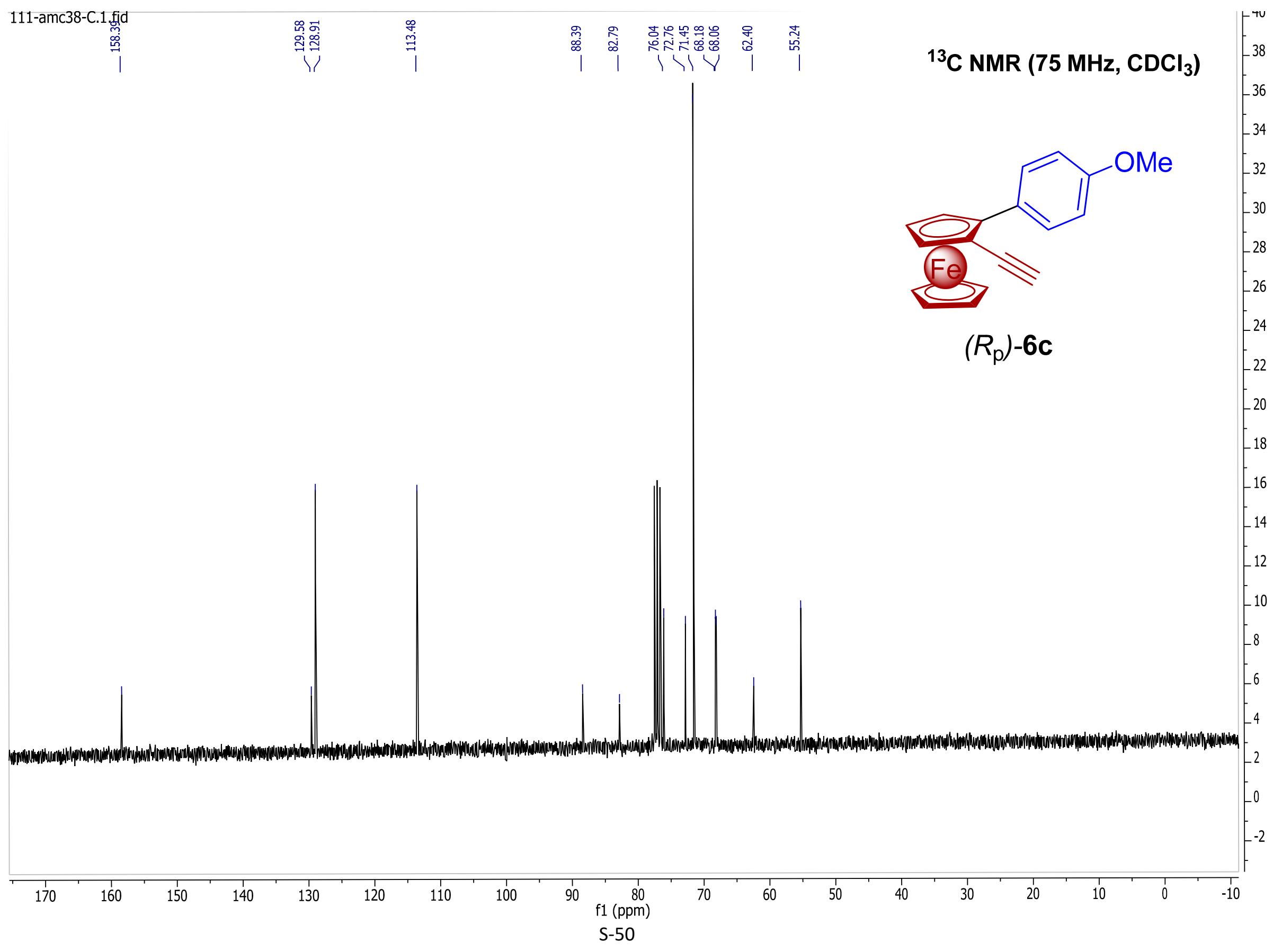




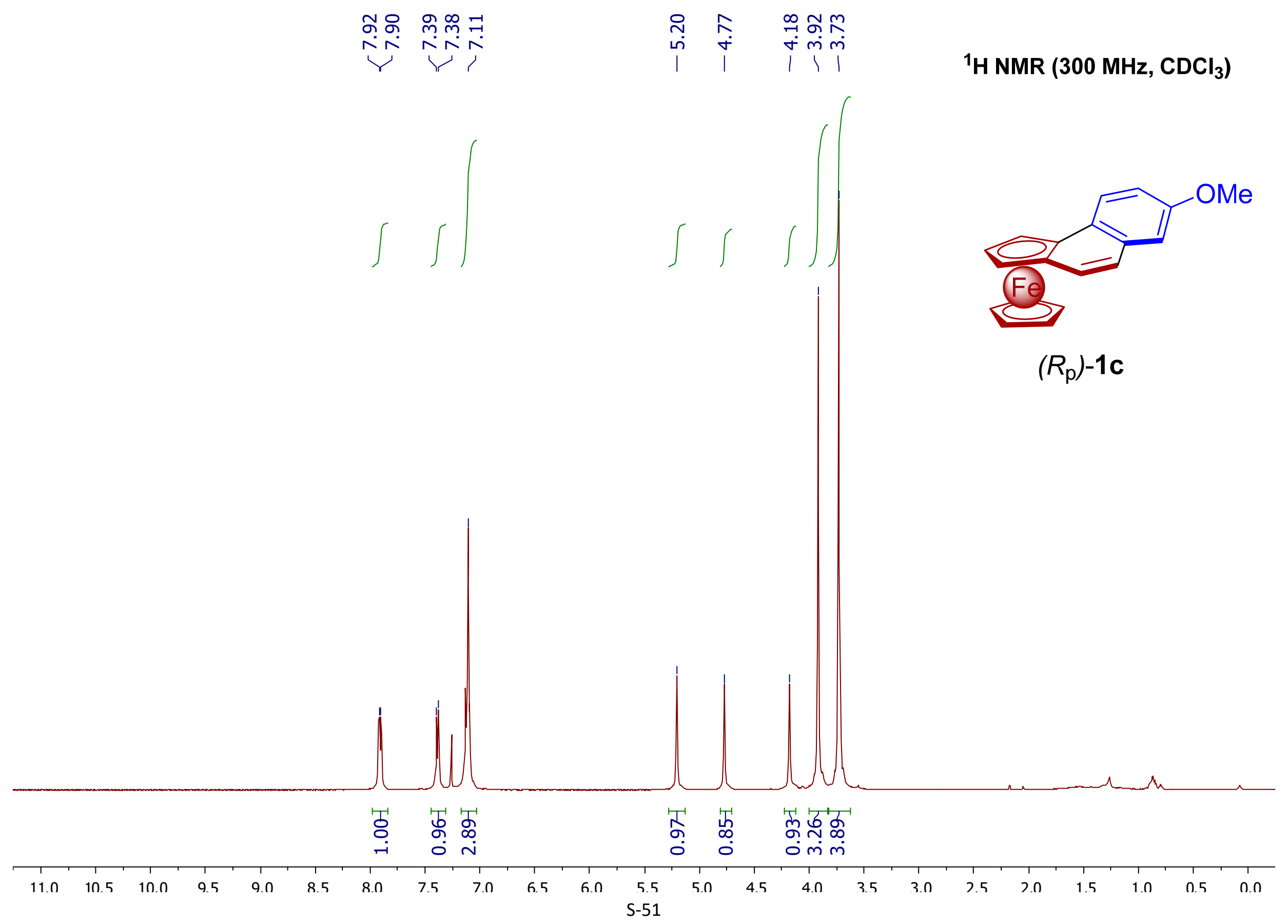



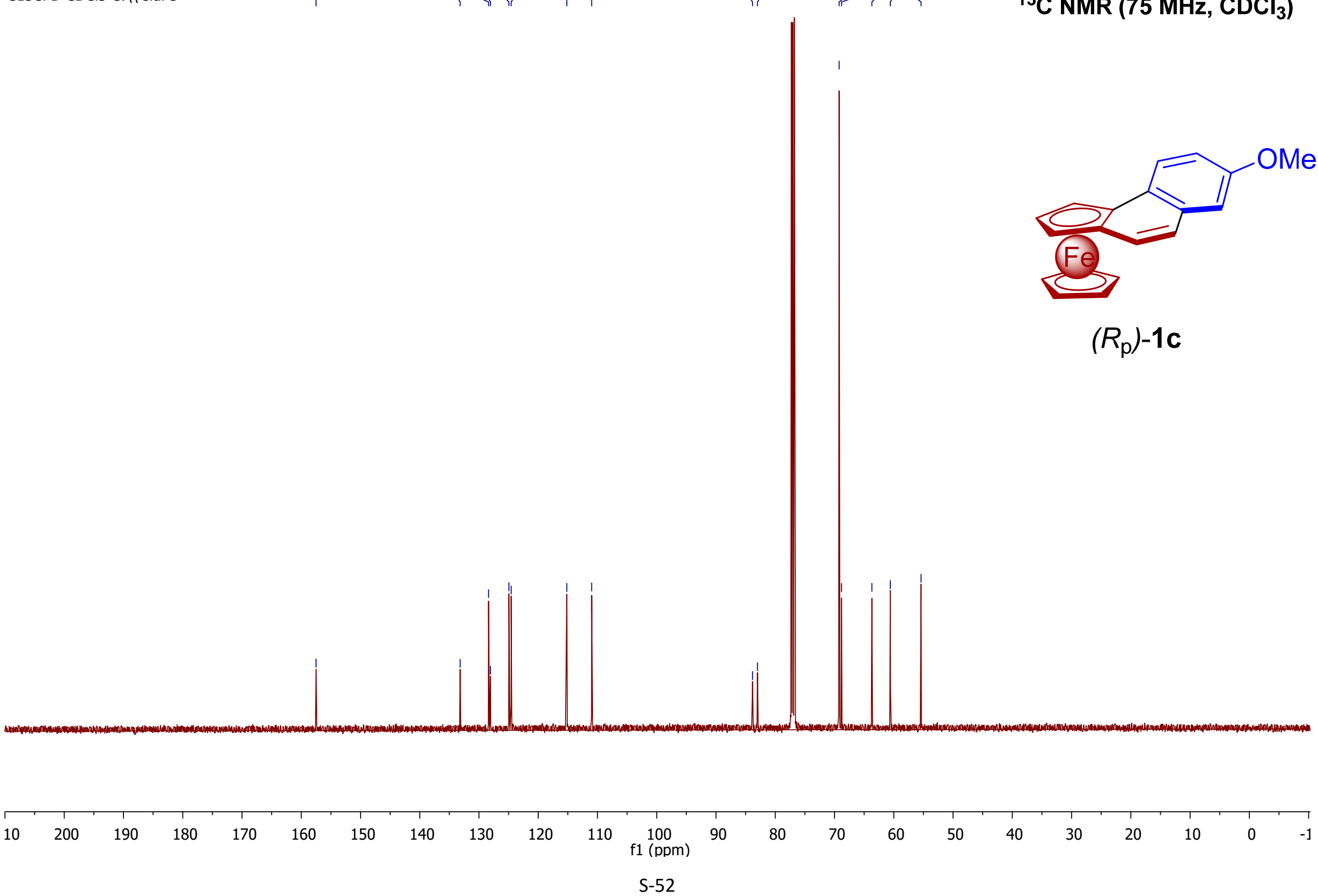


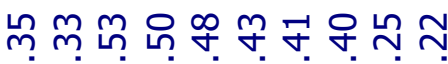

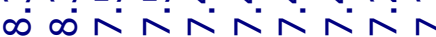

$1 \longrightarrow$

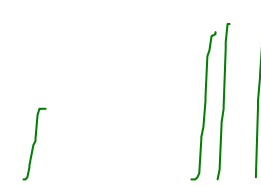

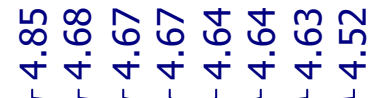

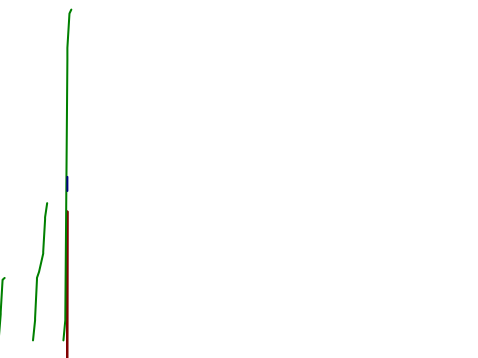

${ }^{1} \mathrm{H}$ NMR $\left(300 \mathrm{MHz}, \mathrm{CDCl}_{3}\right)$

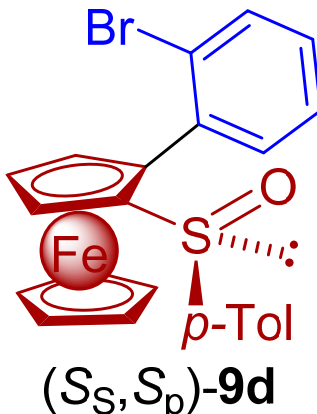

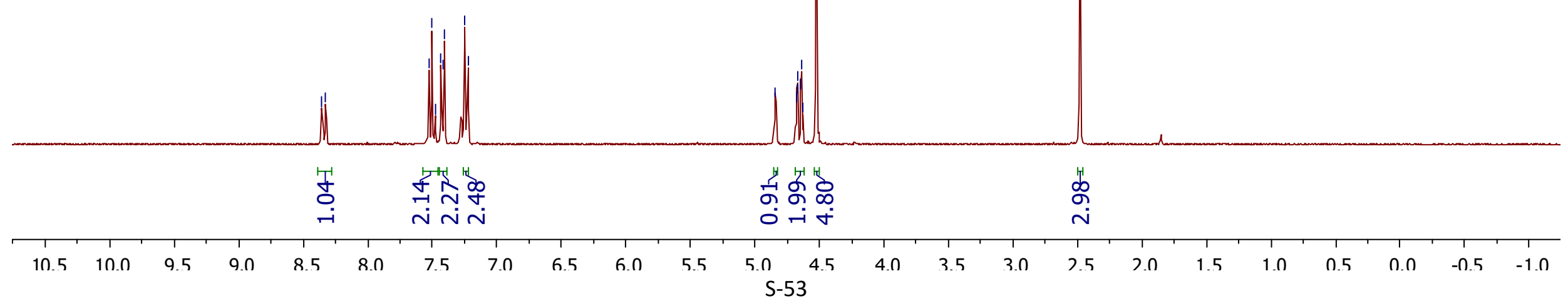




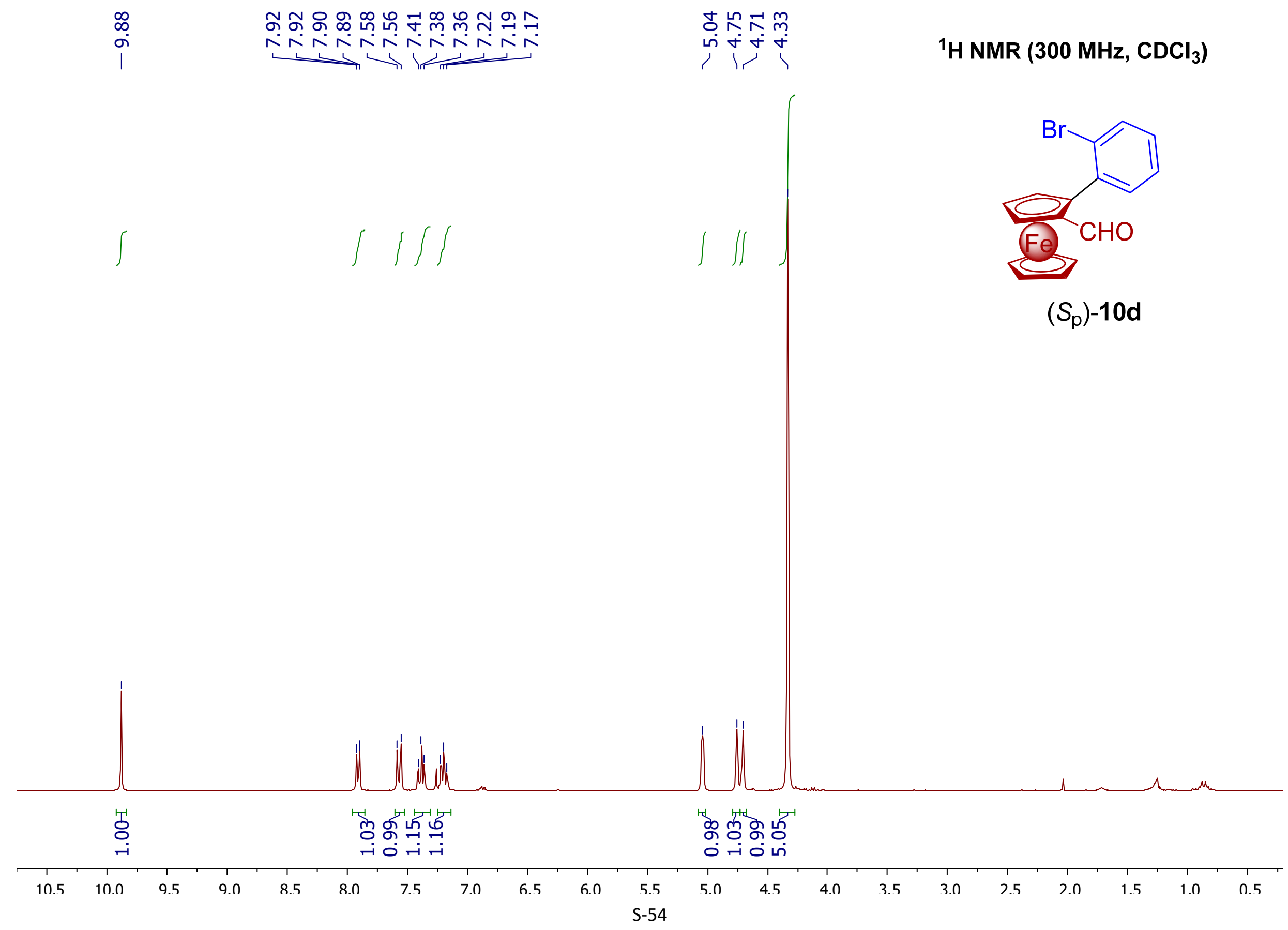




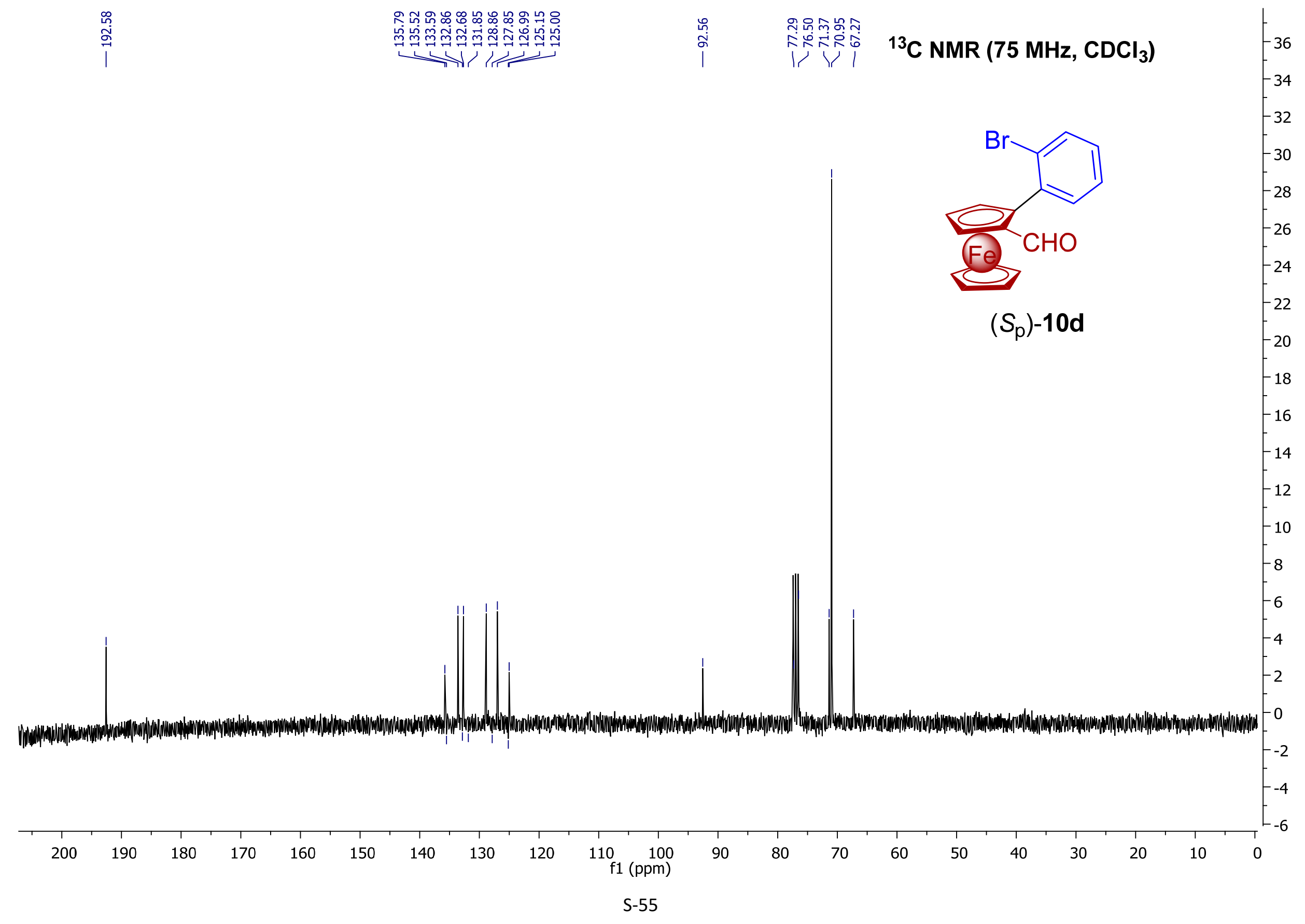




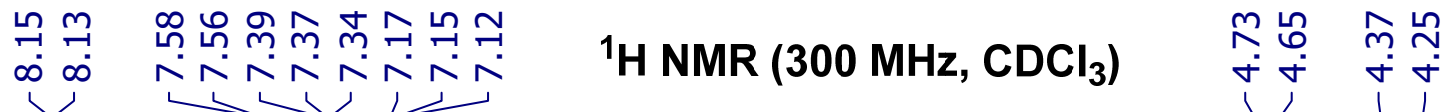

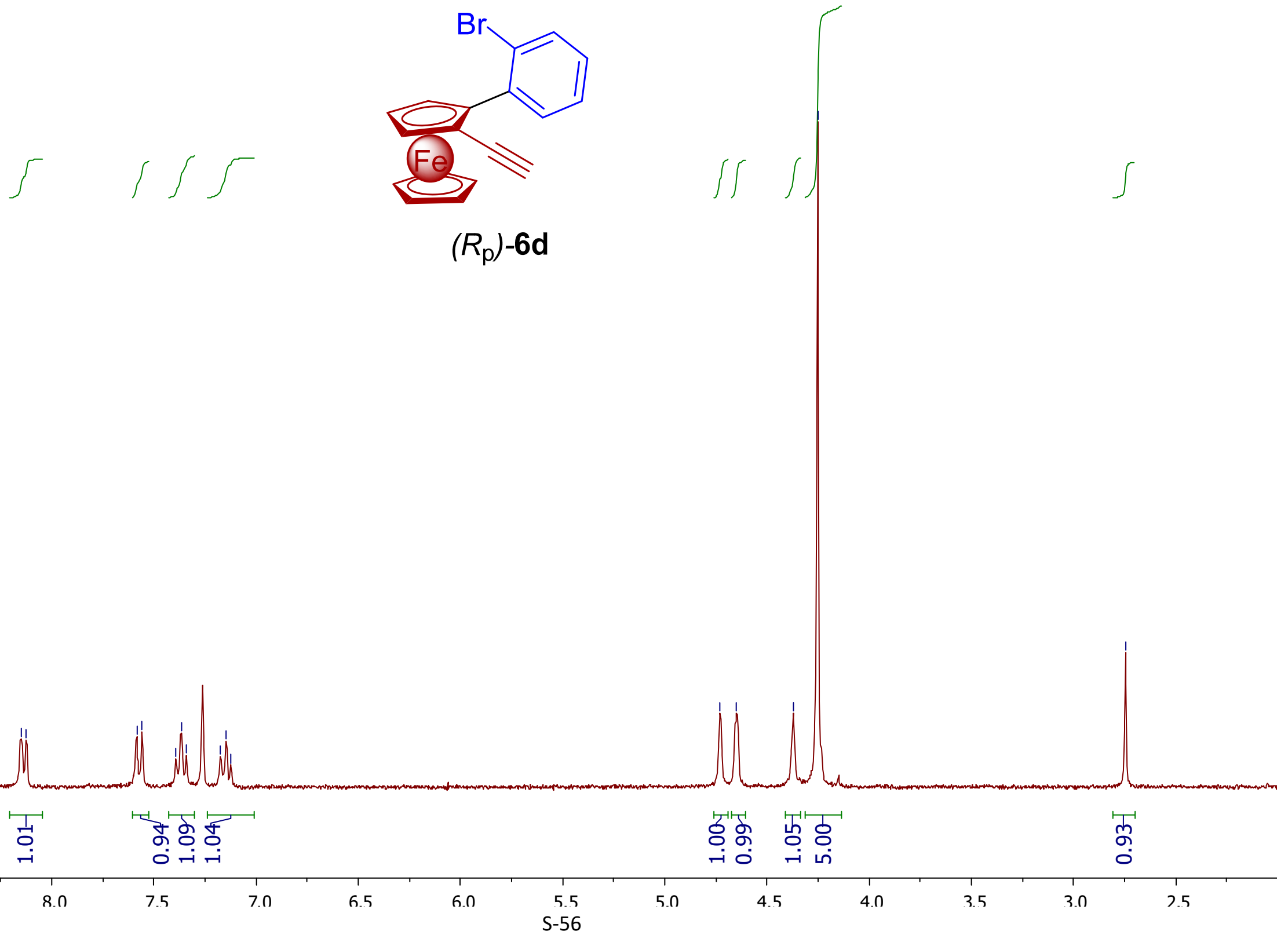




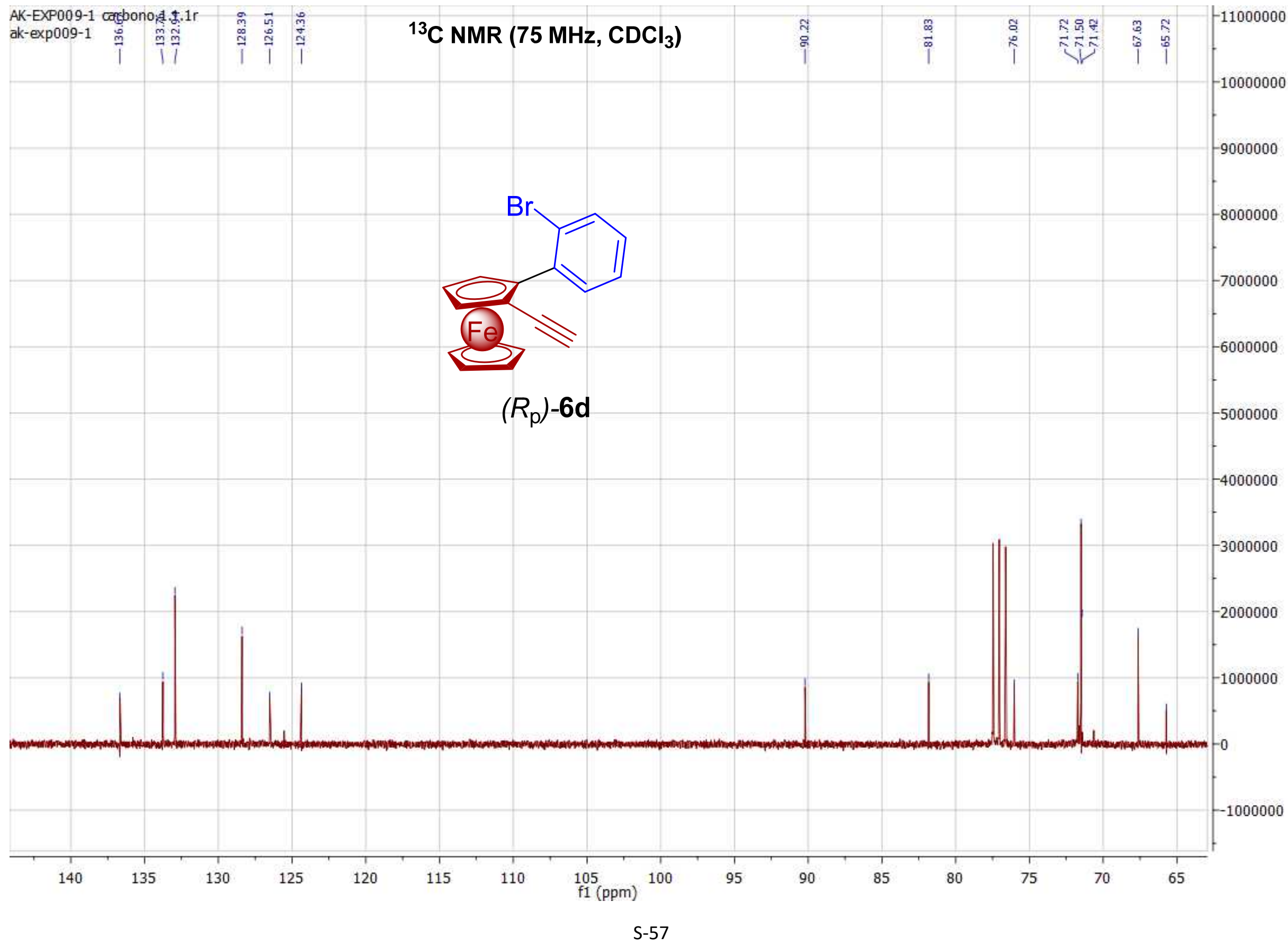




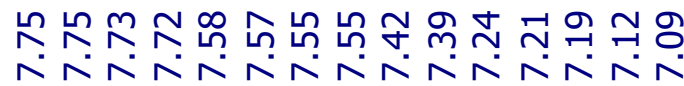

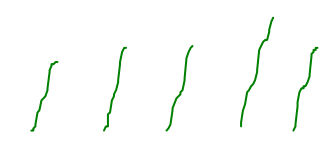

$\vec{m}$ ๒ै ${ }^{1} \mathrm{H}$ NMR (300 MHz, $\mathrm{CDCl}_{3}$ )

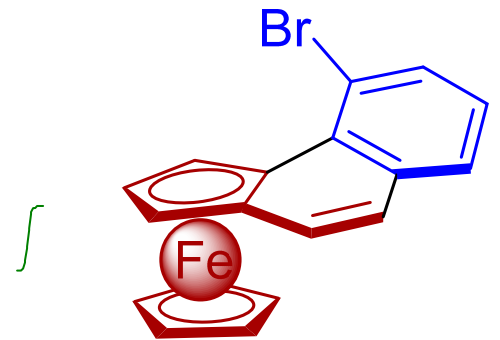

$\left(R_{\mathrm{p}}\right)-1 \mathrm{~d}$

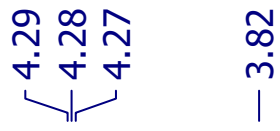

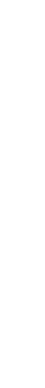

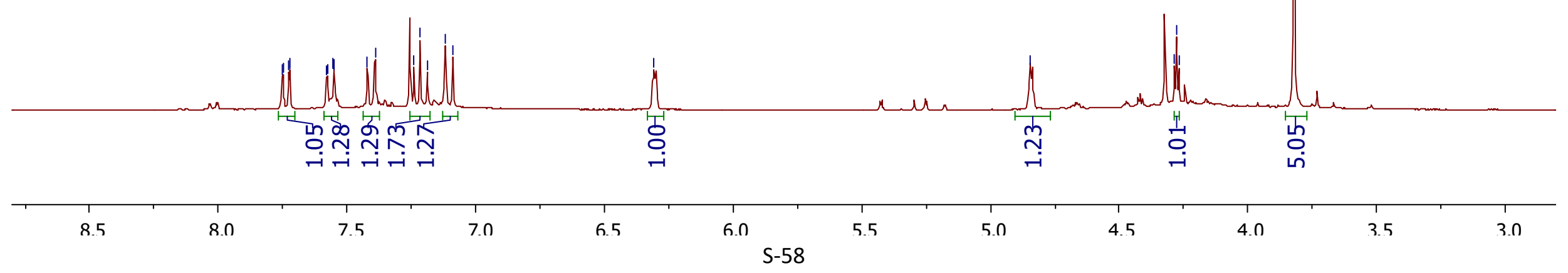




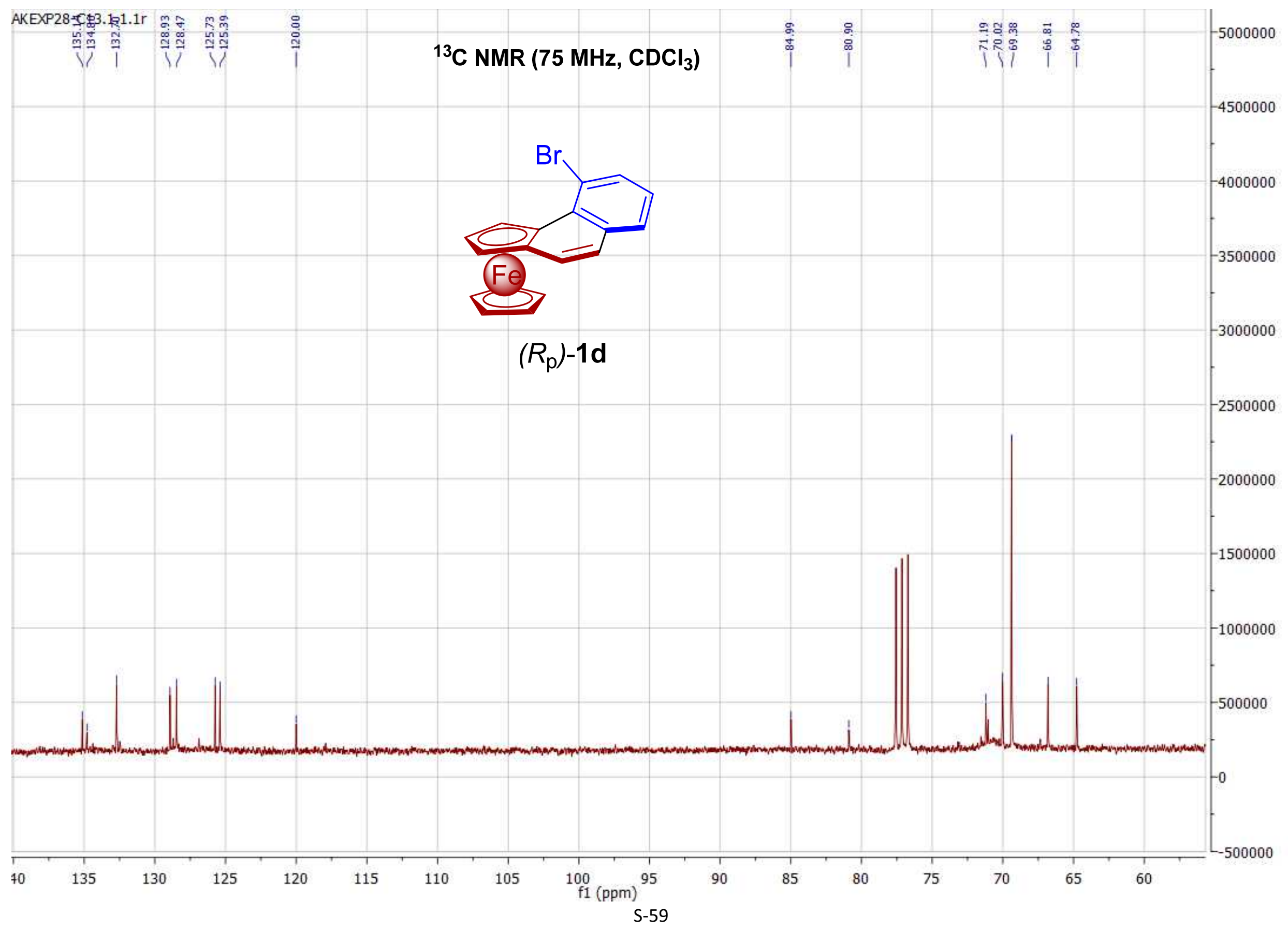




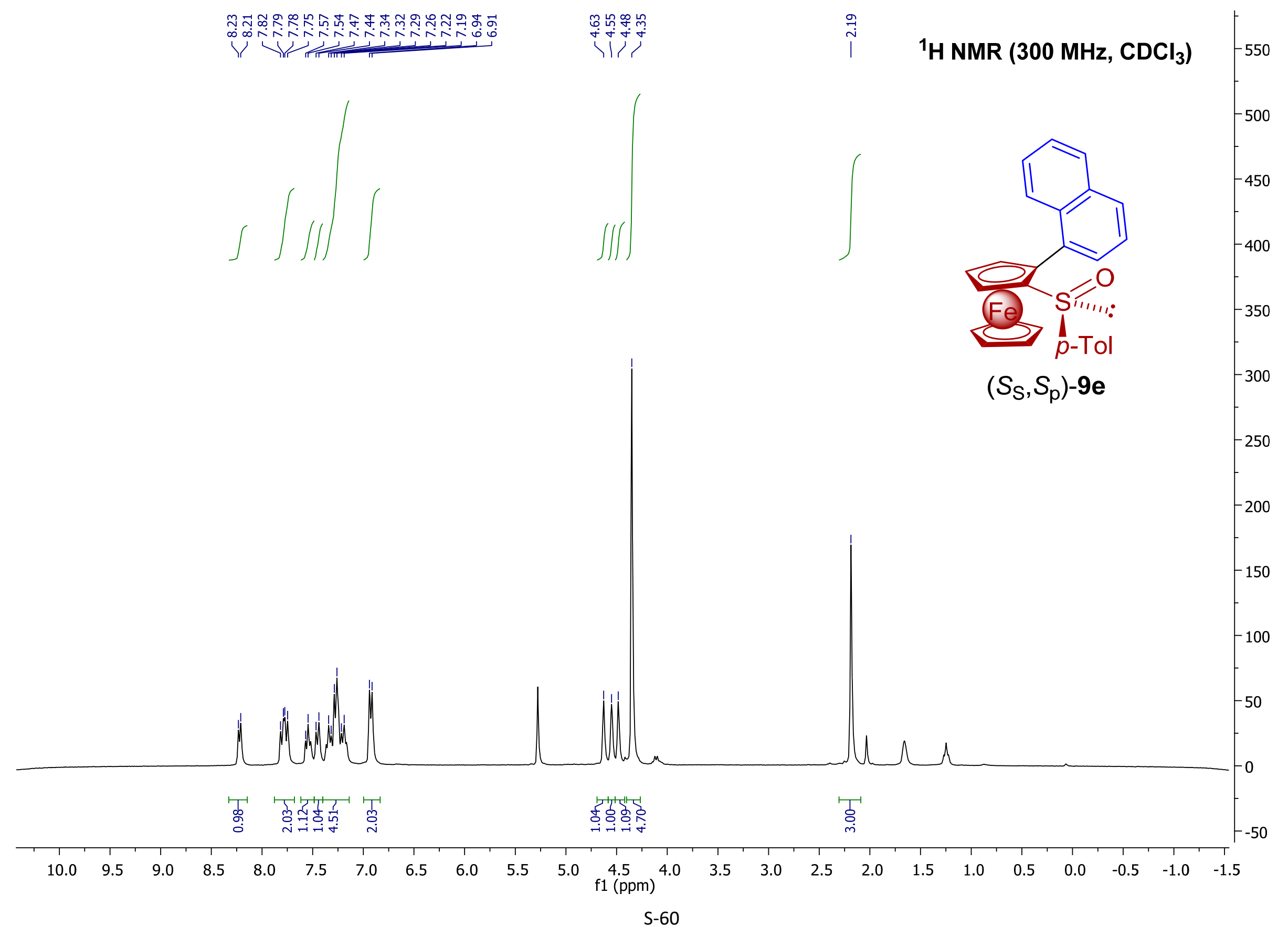




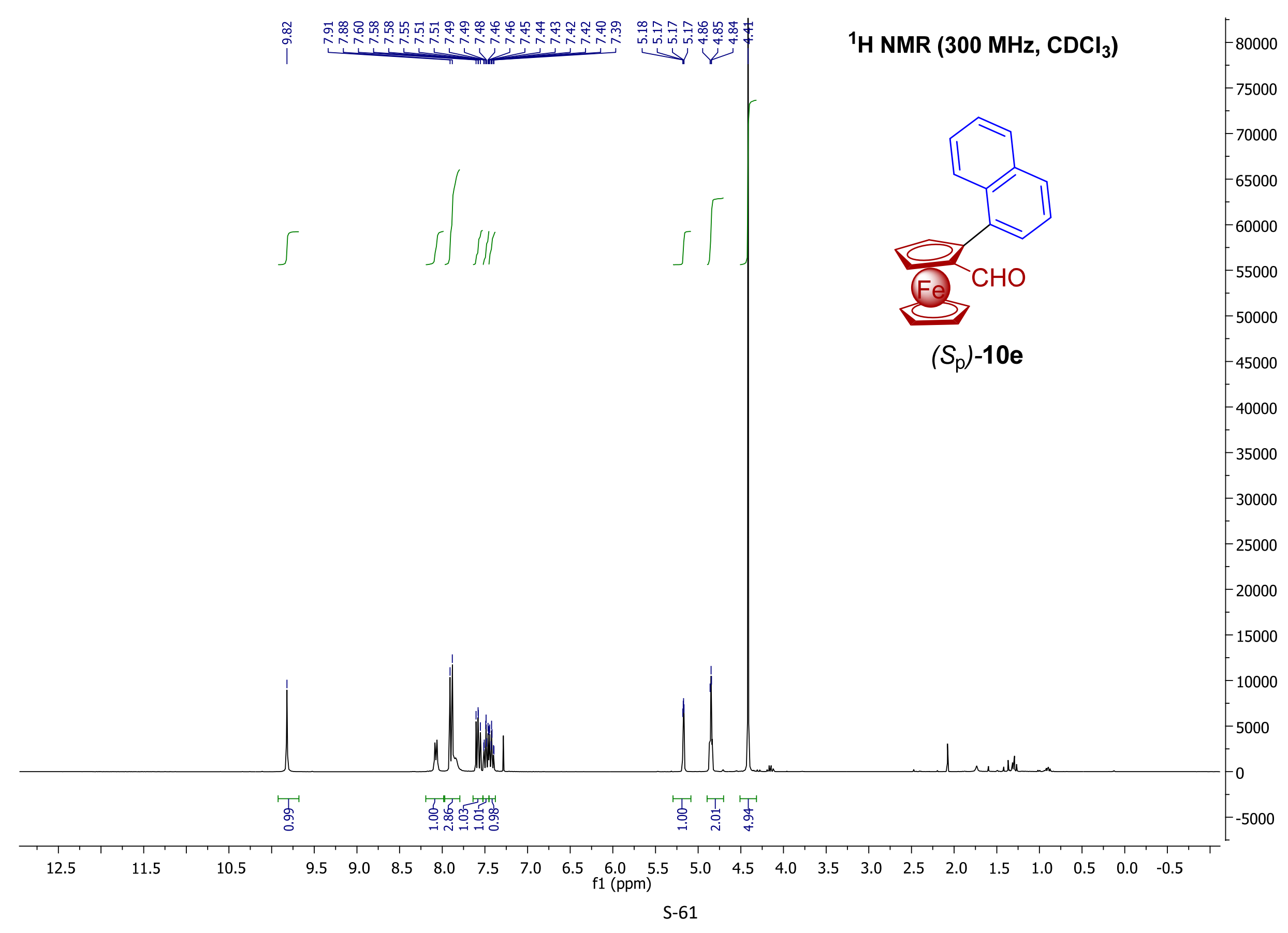




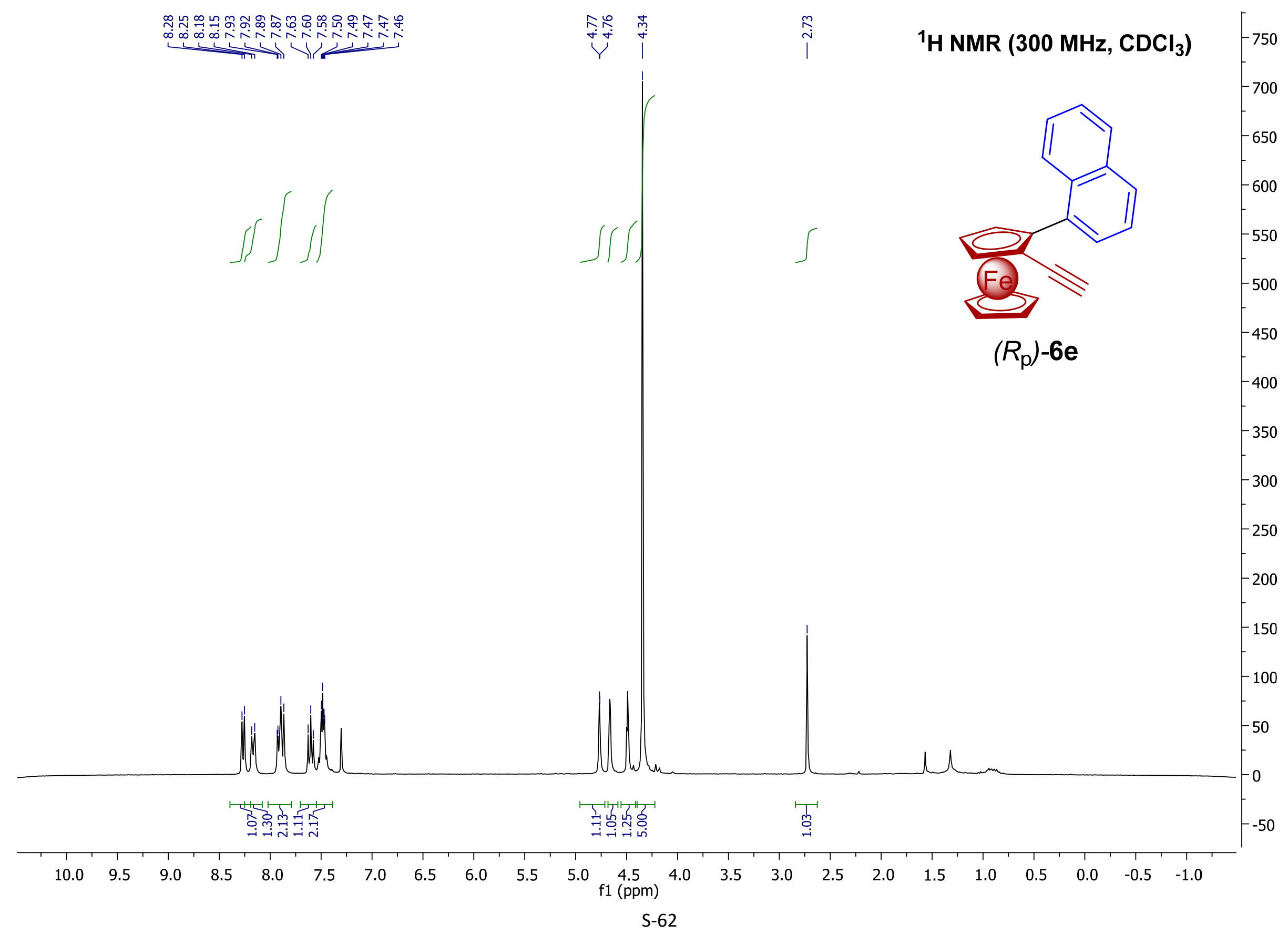




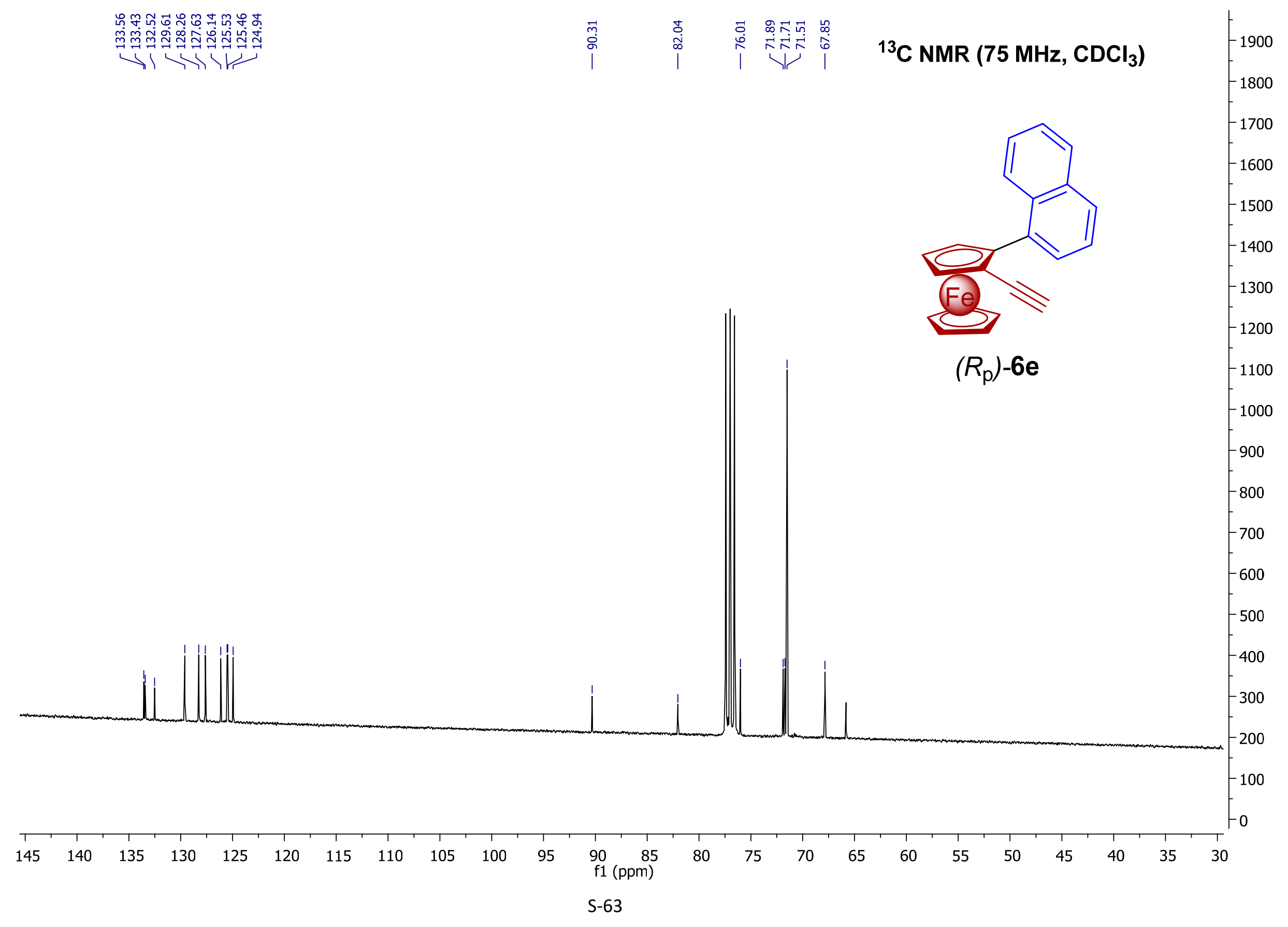




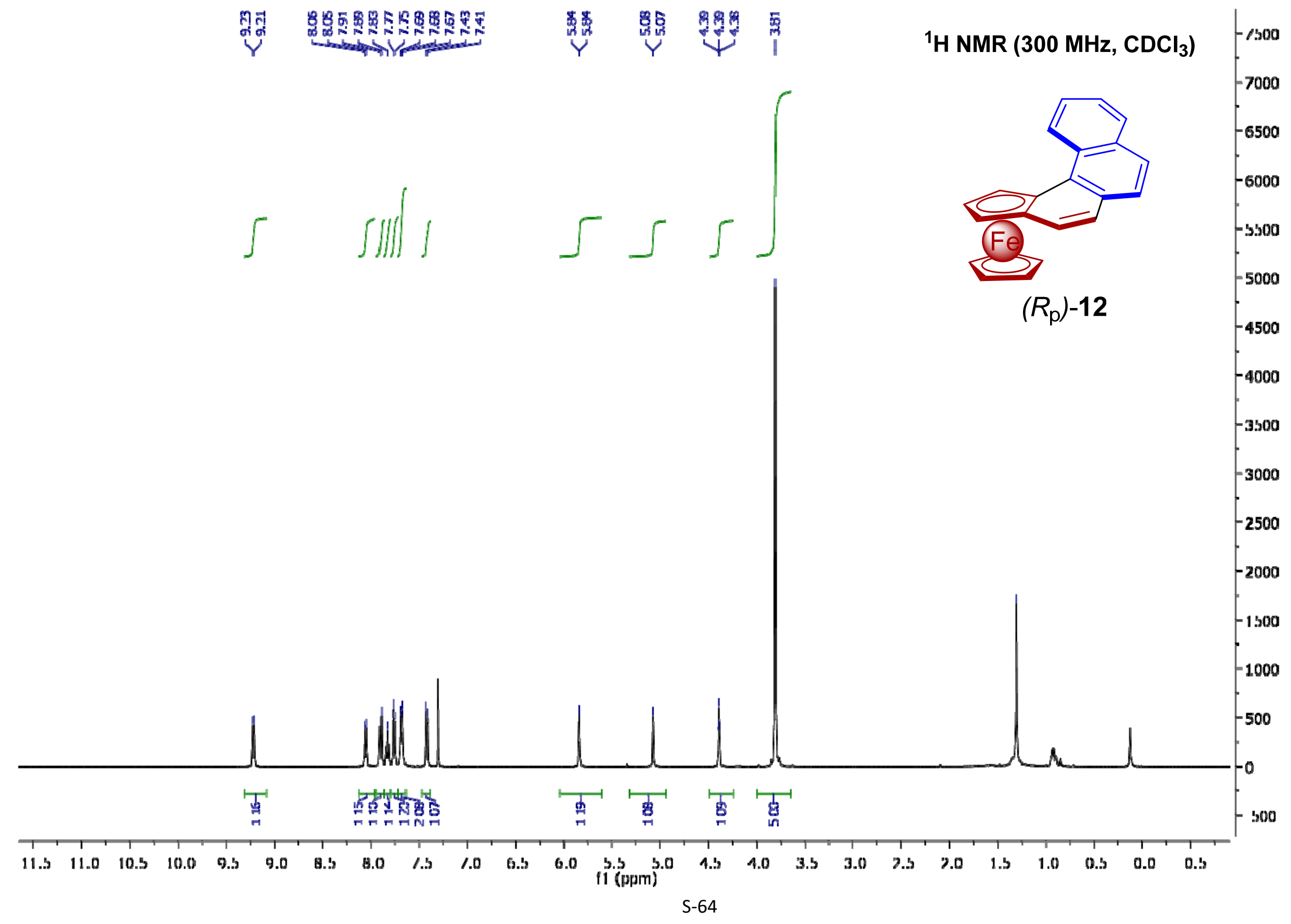




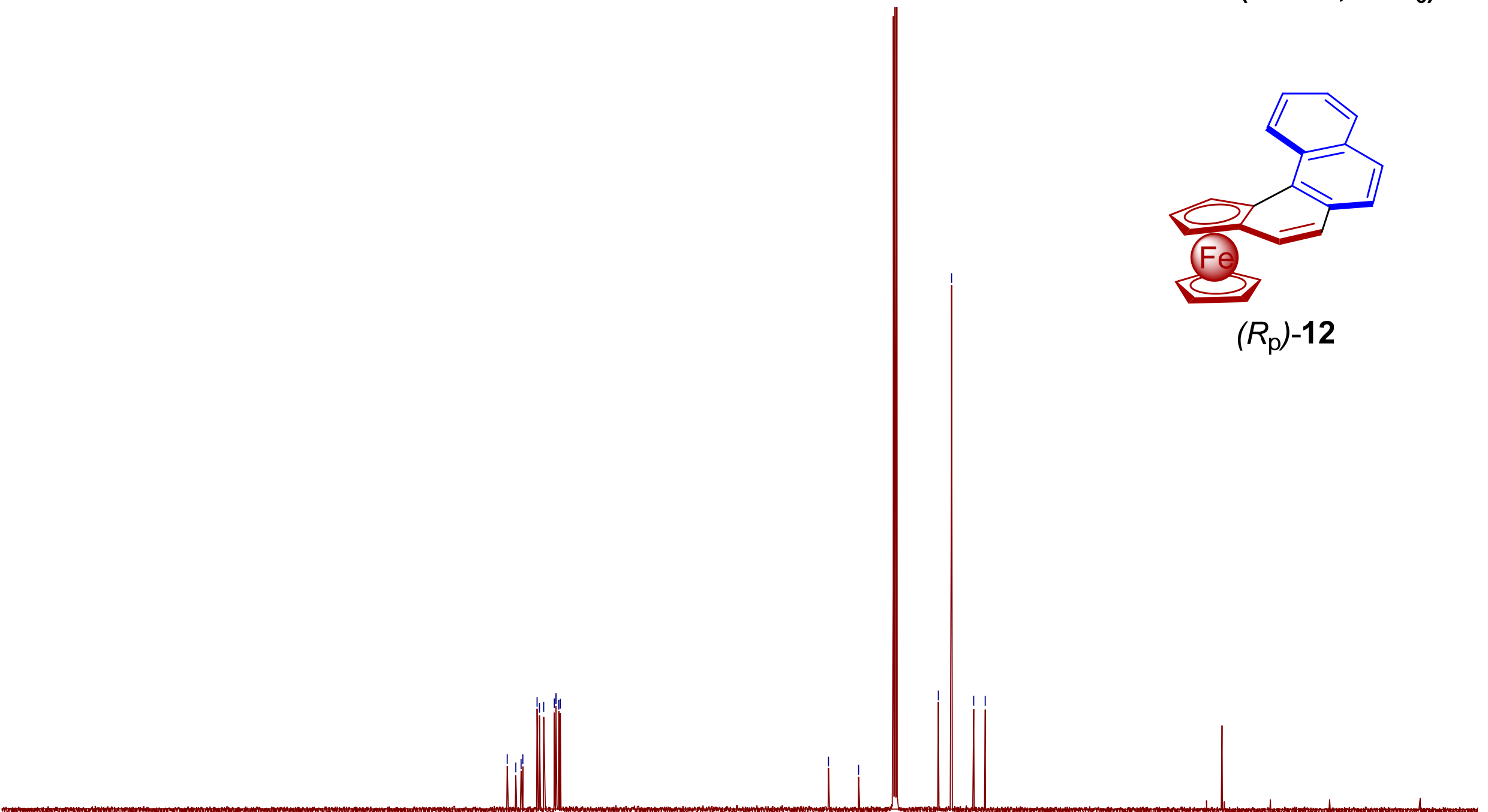

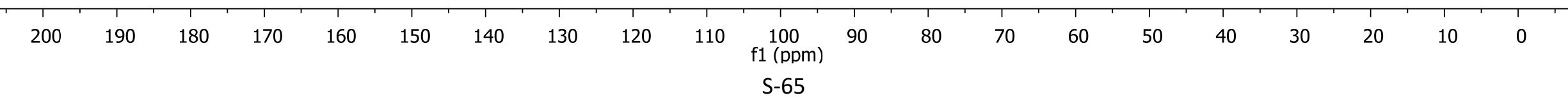




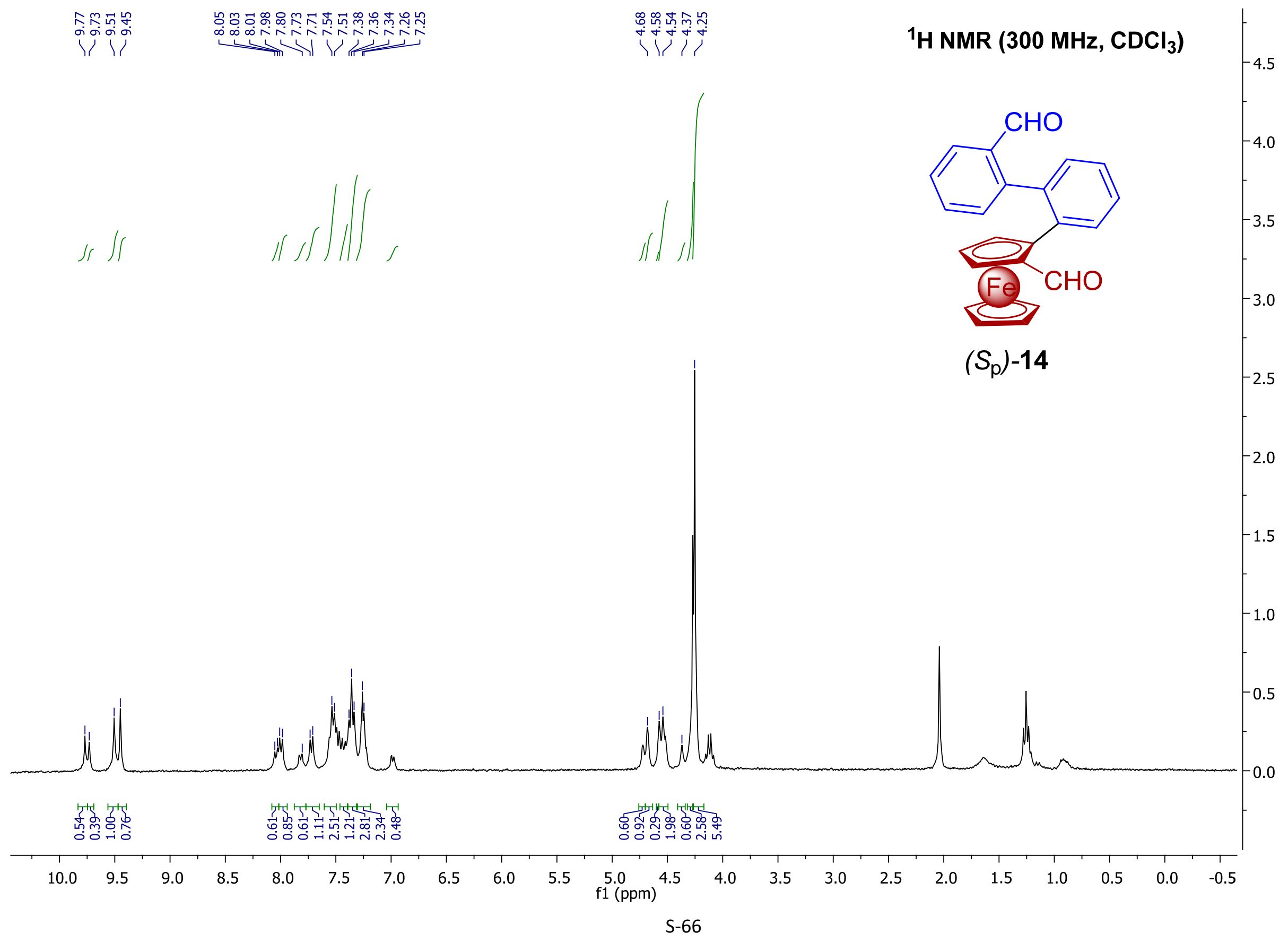




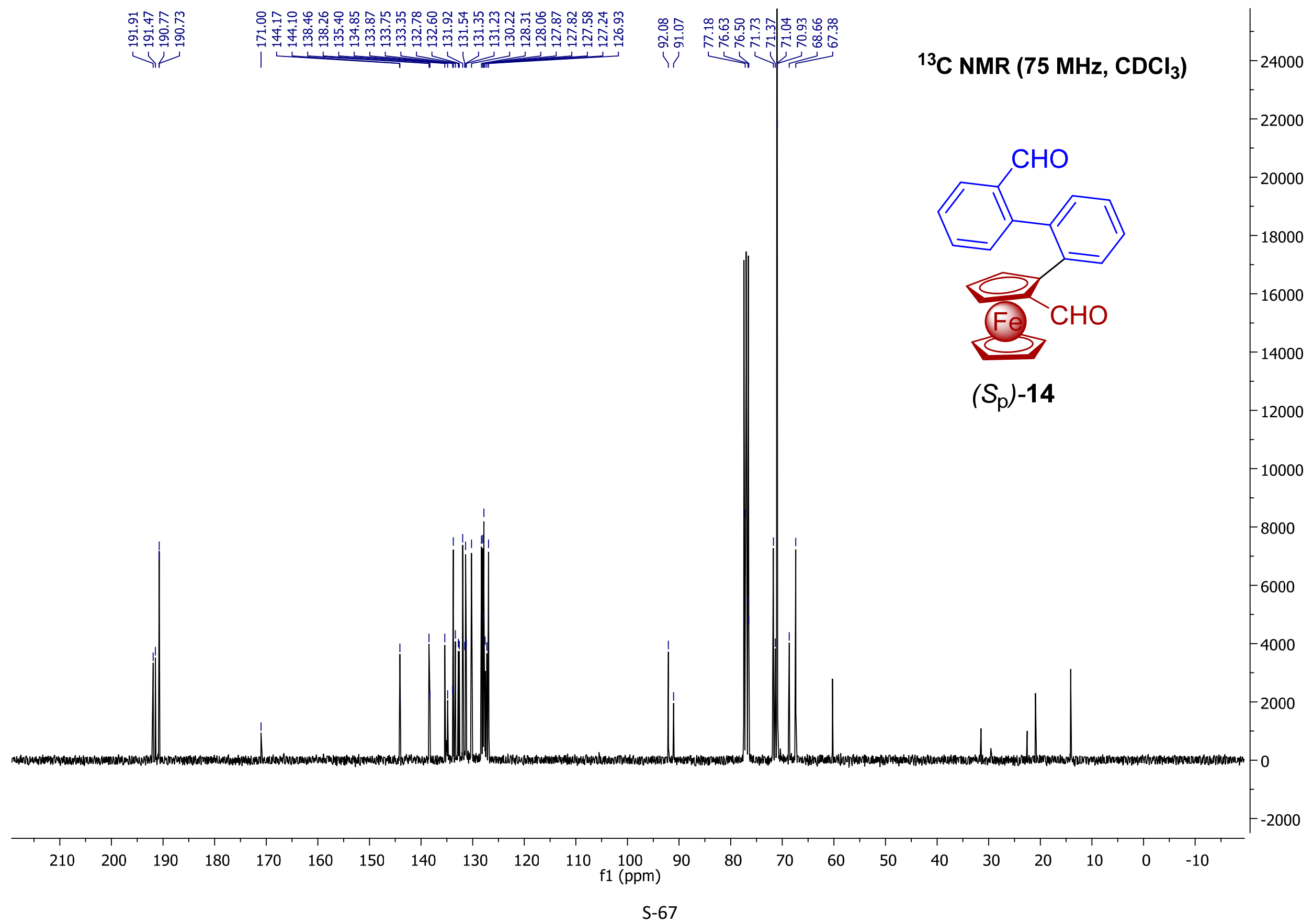




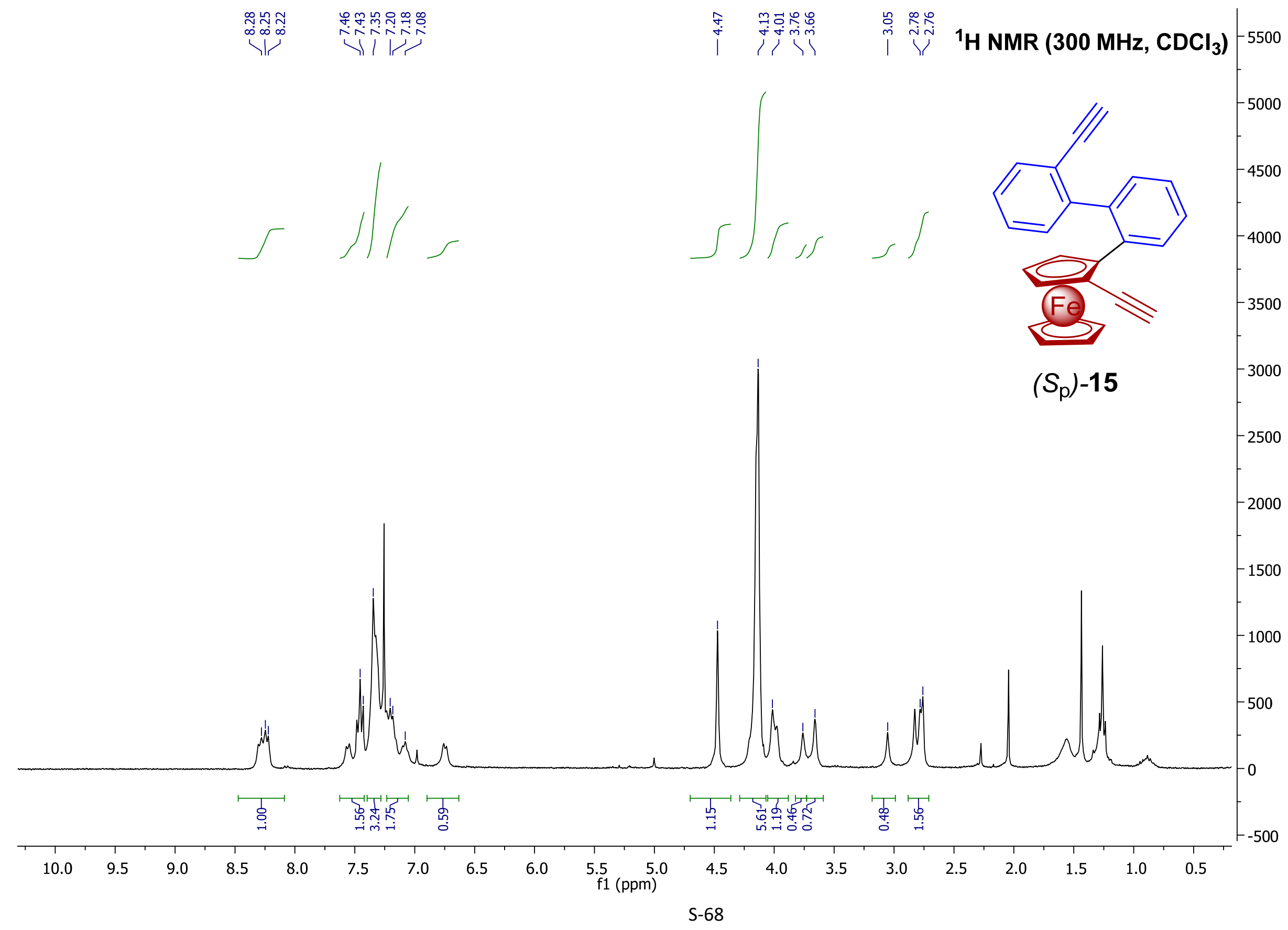




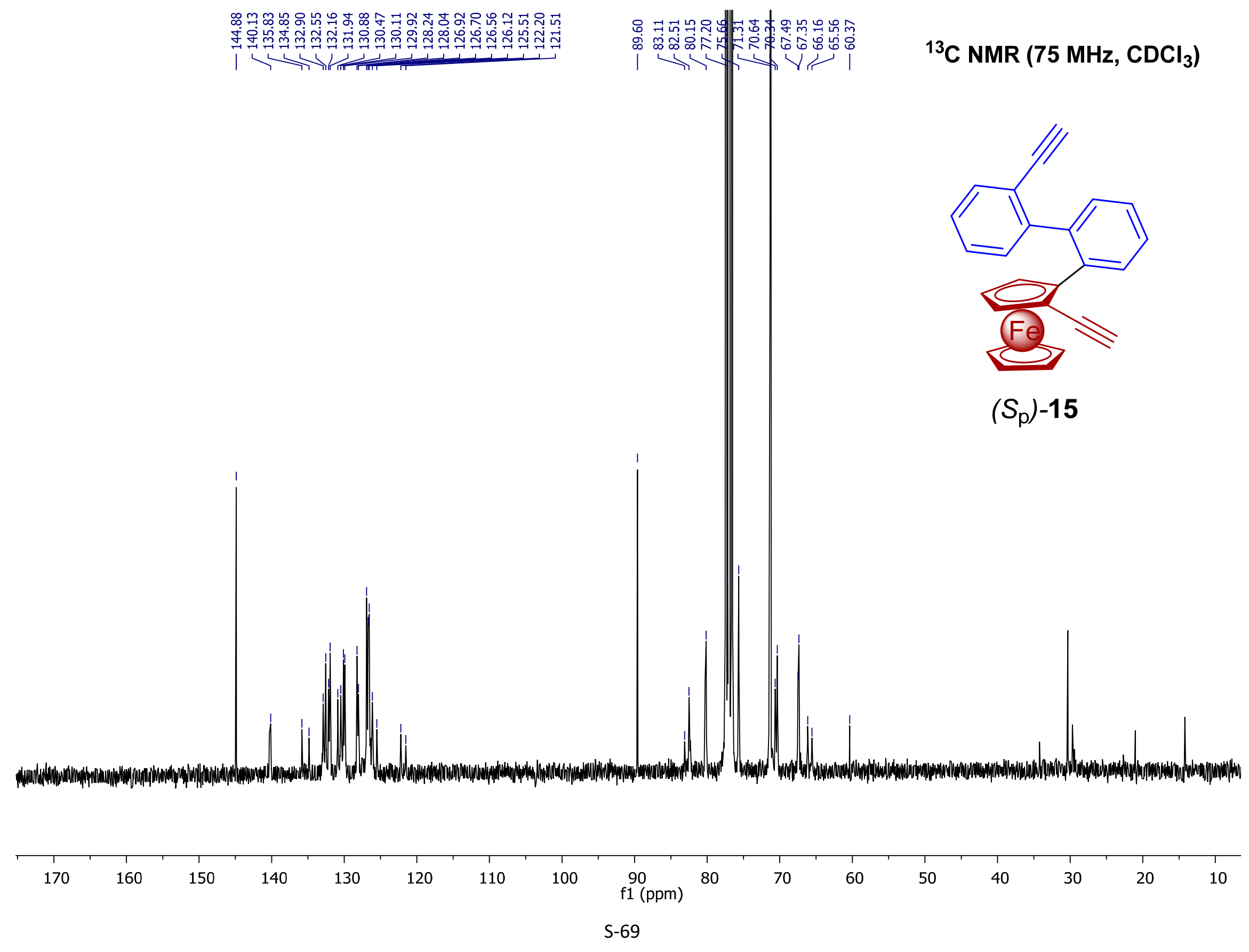




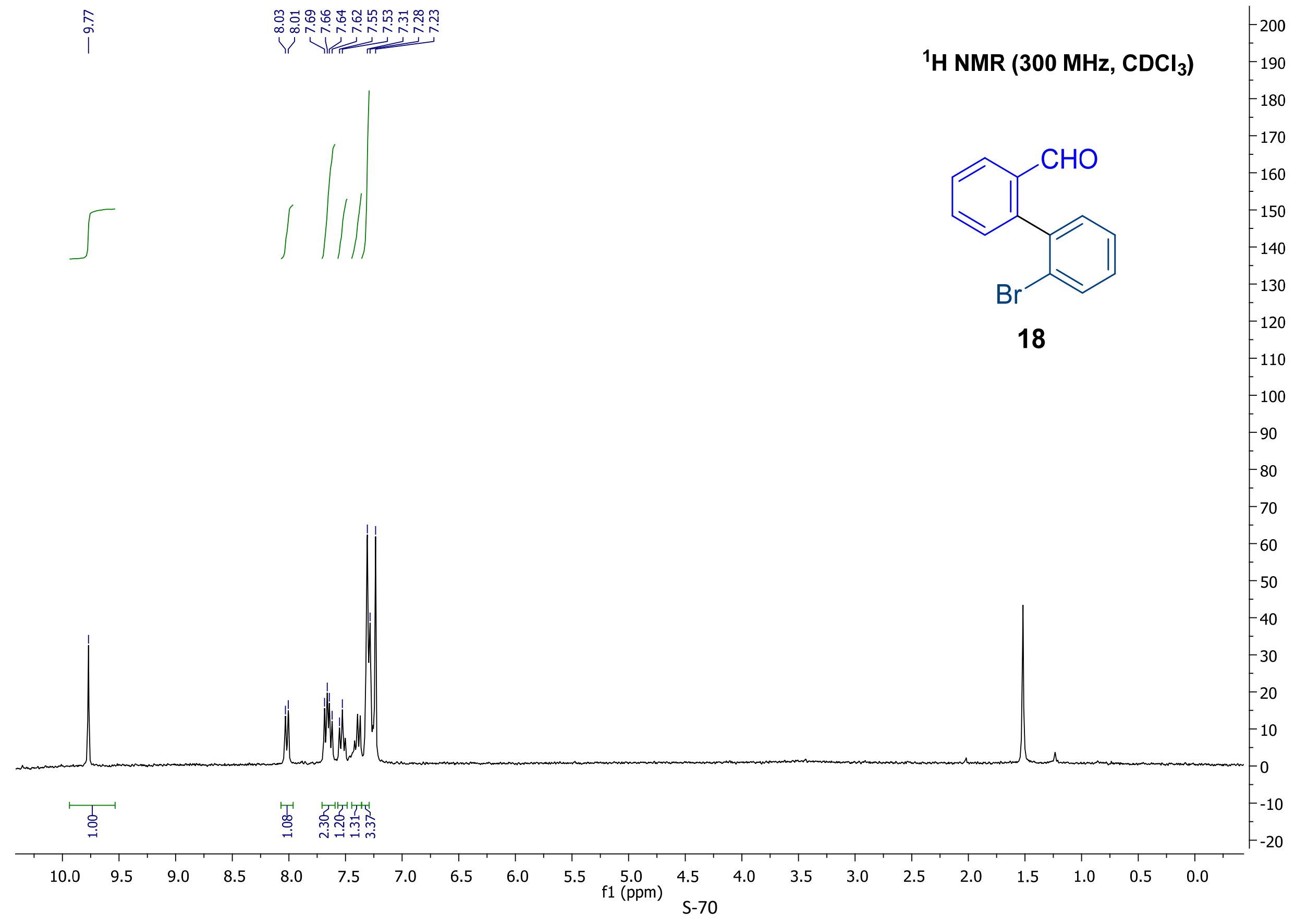




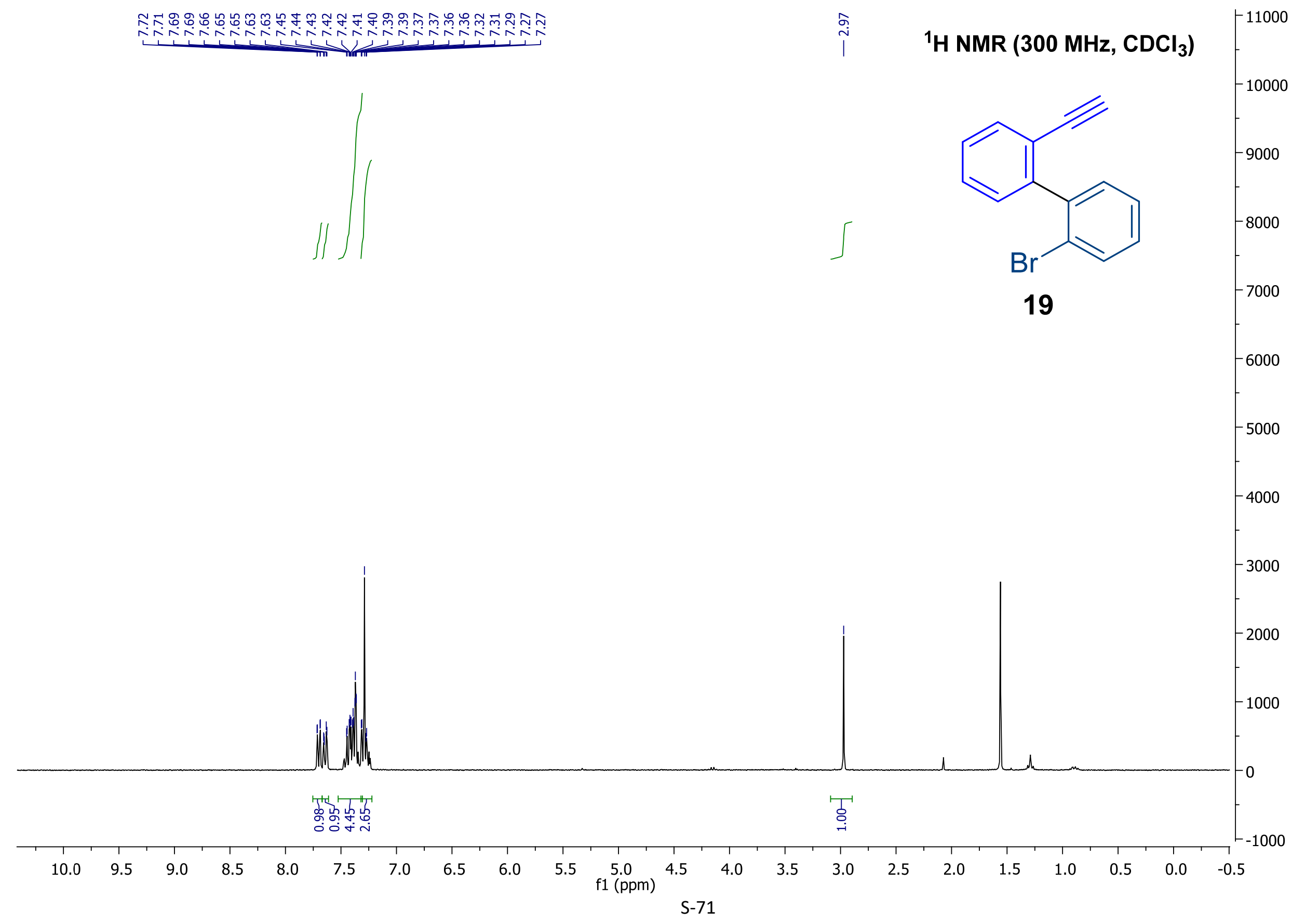




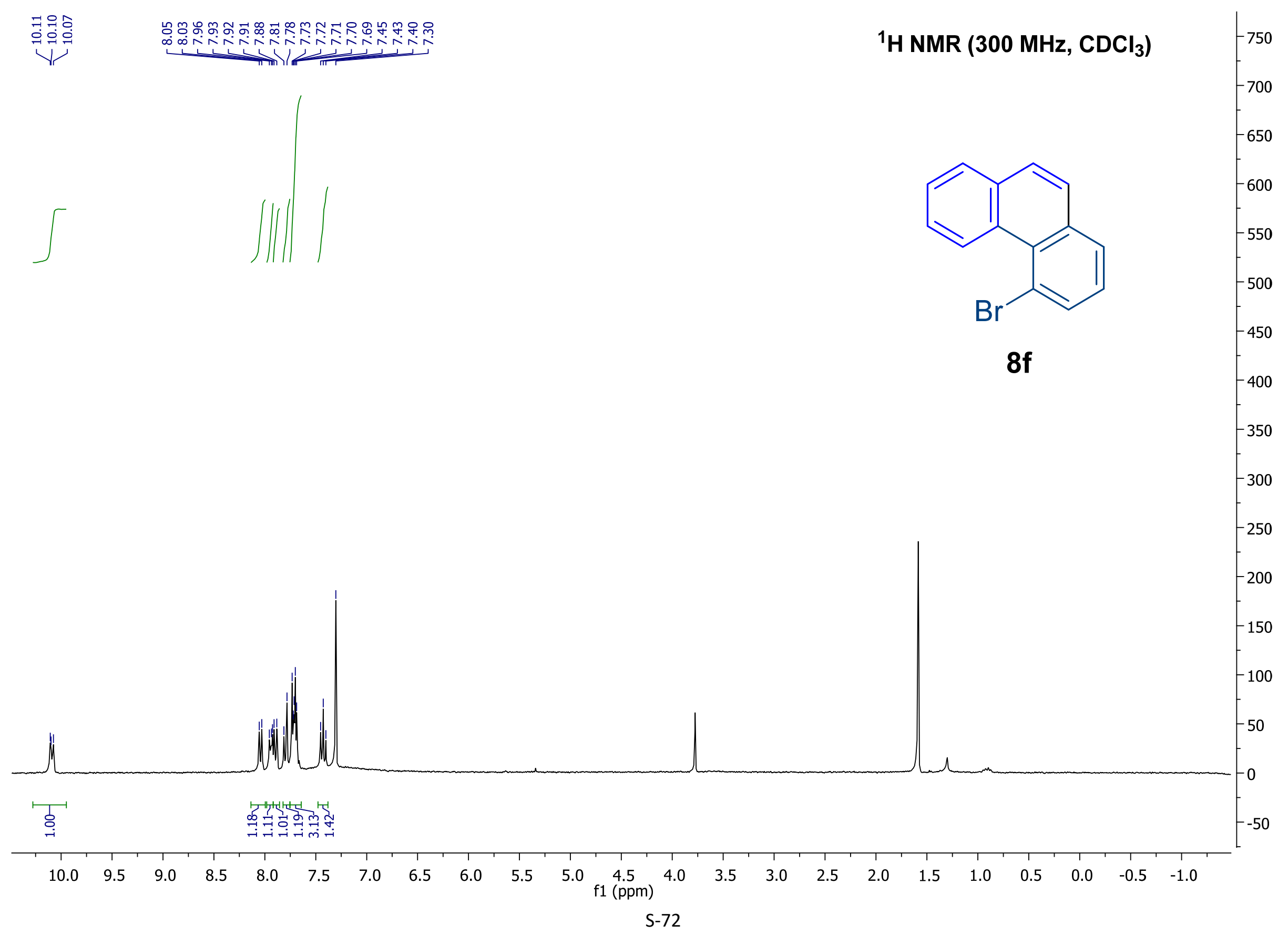




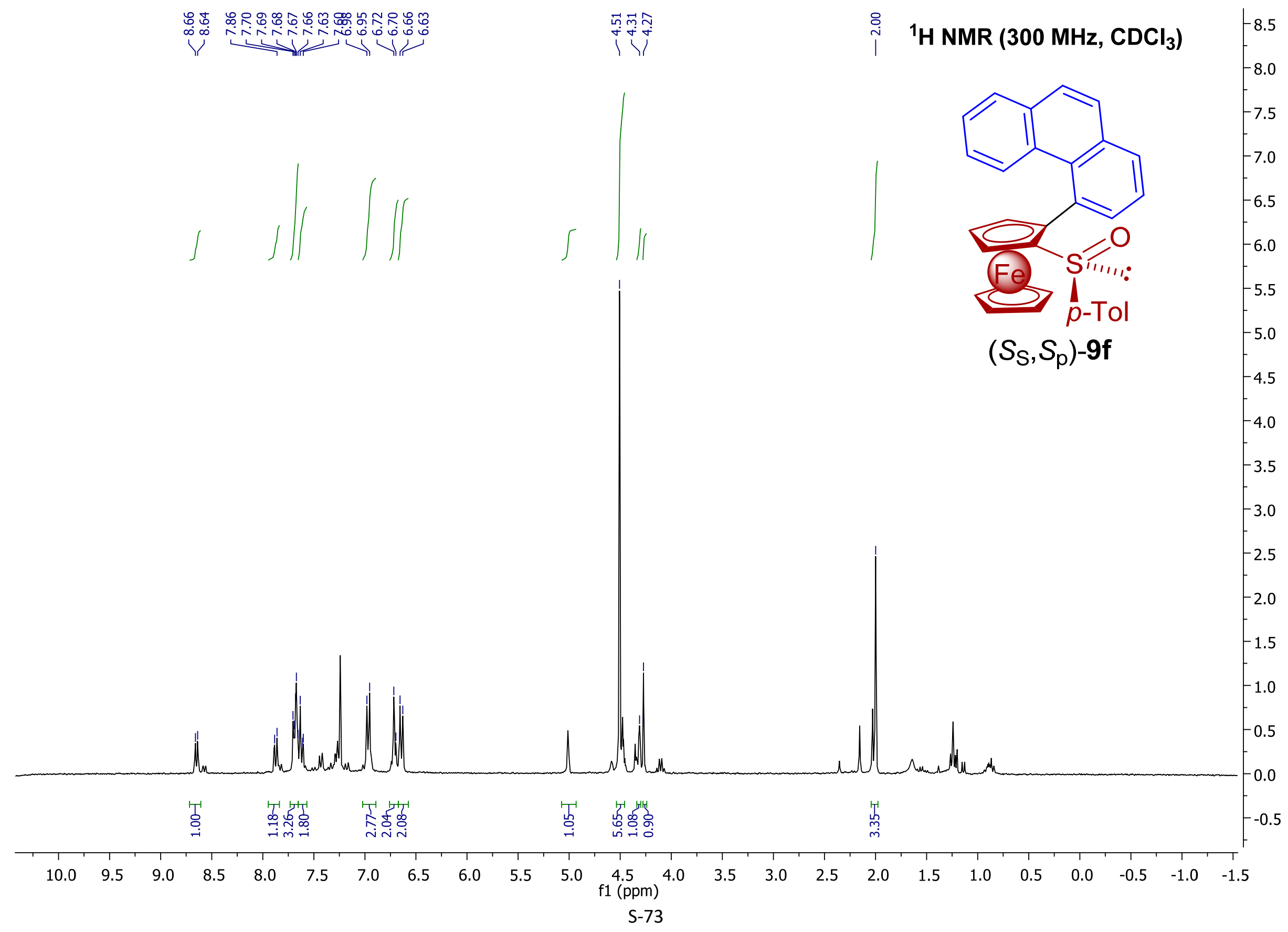


$\left.{ }^{13} \mathrm{C} \mathrm{NMR} \mathrm{(75} \mathrm{MHz,} \mathrm{CDCl}_{3}\right)$

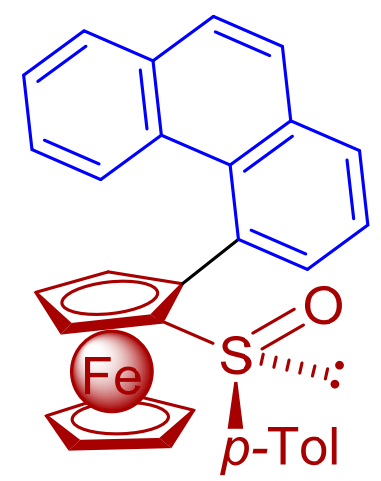




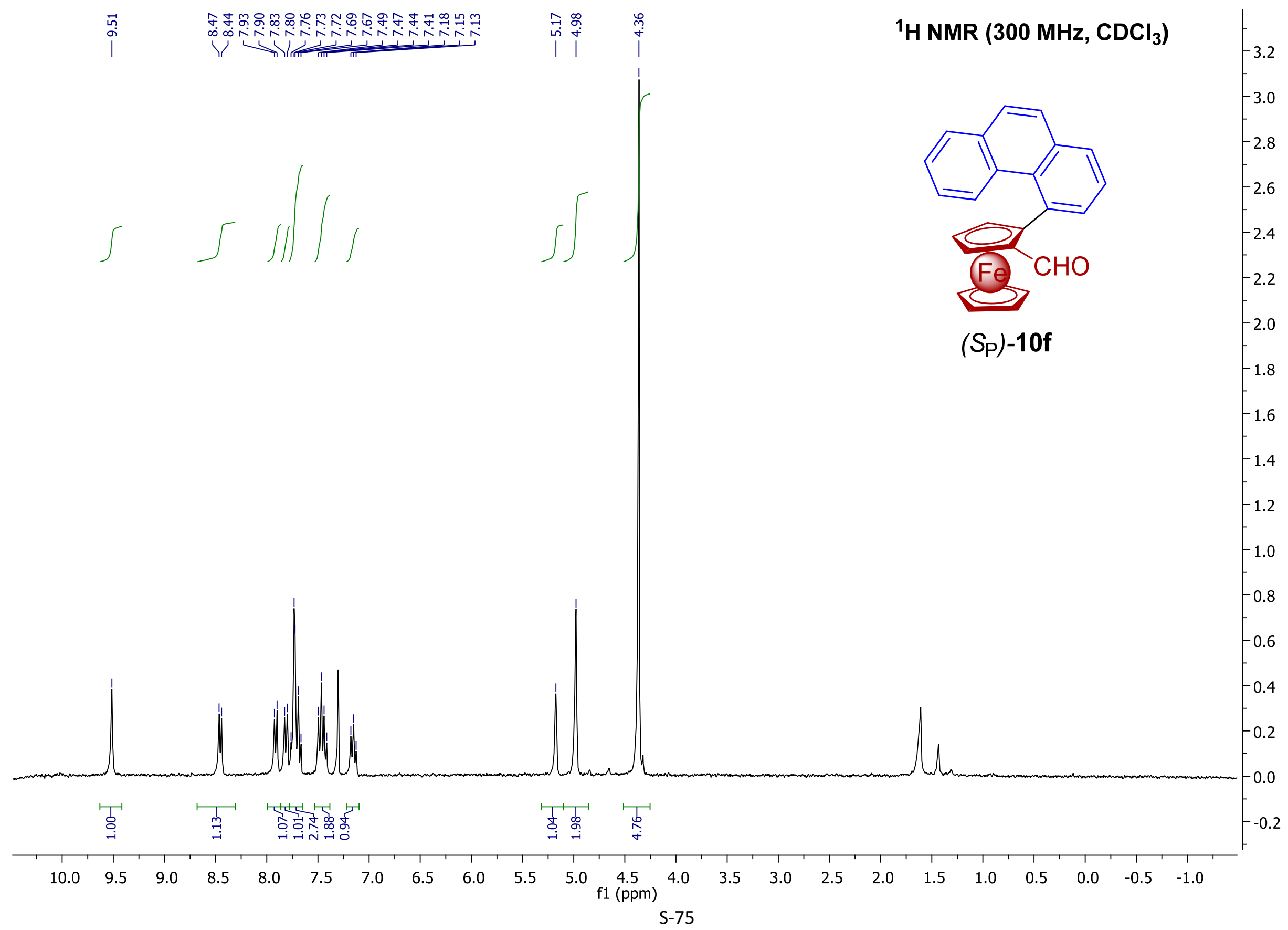




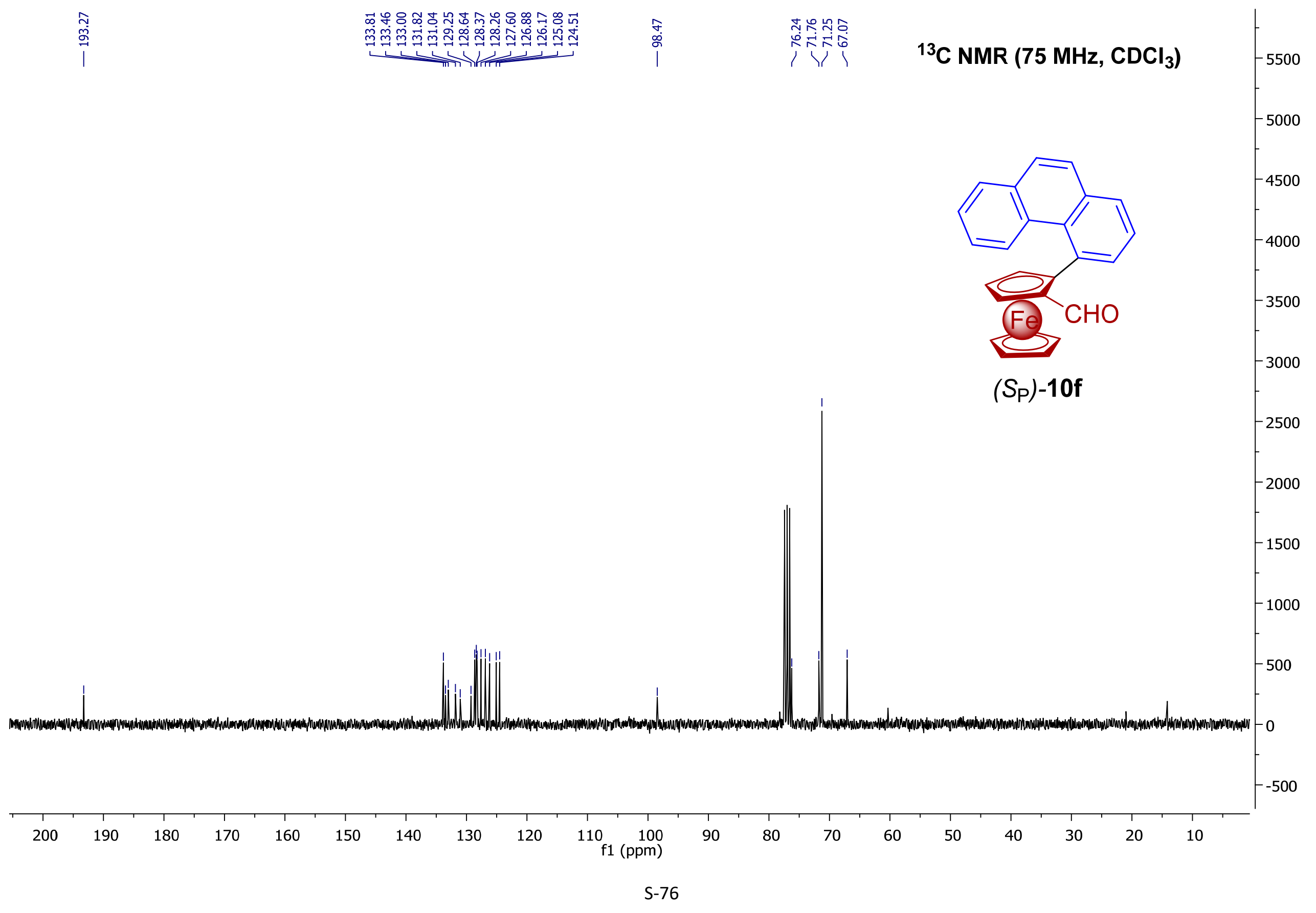




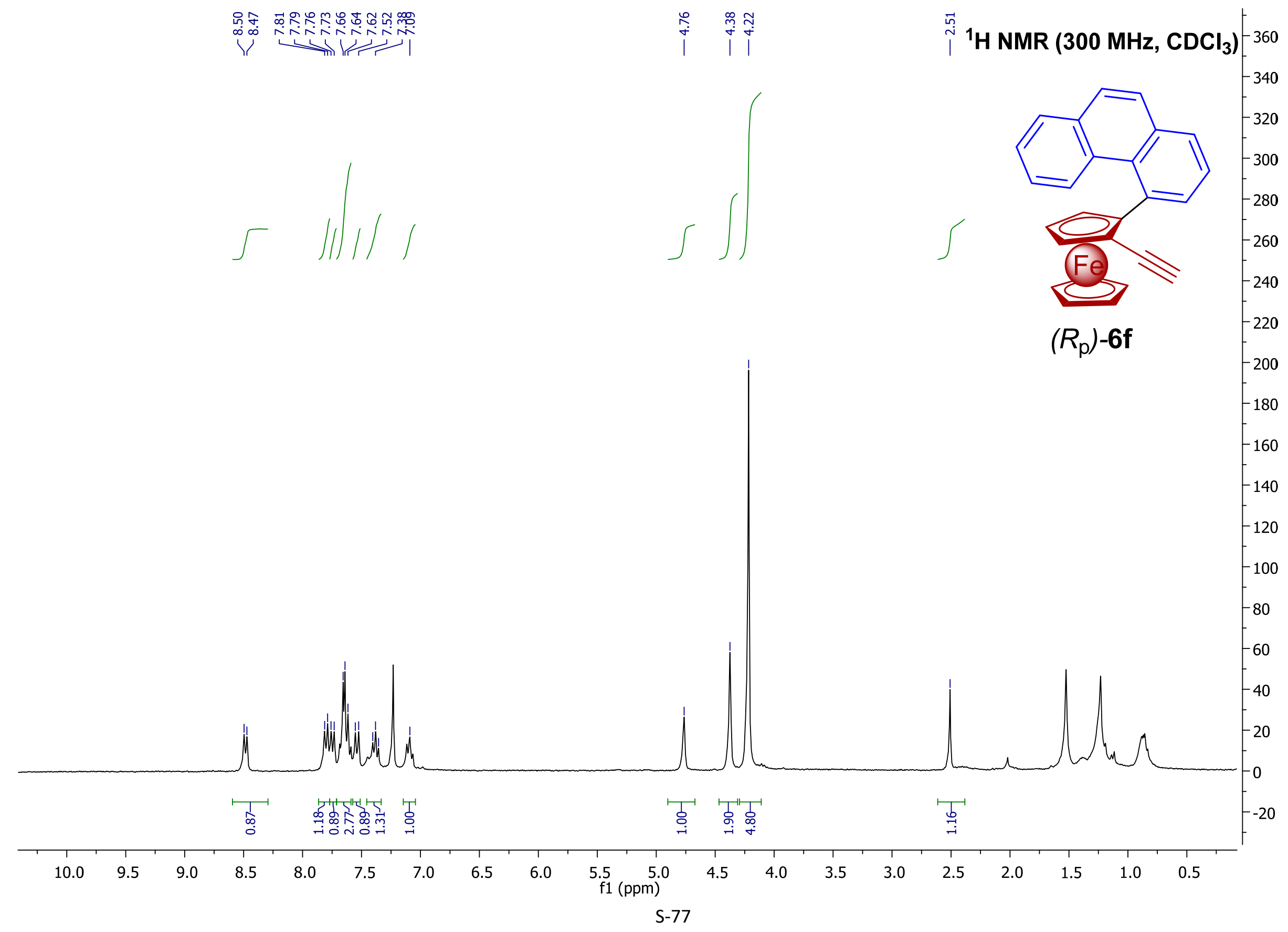




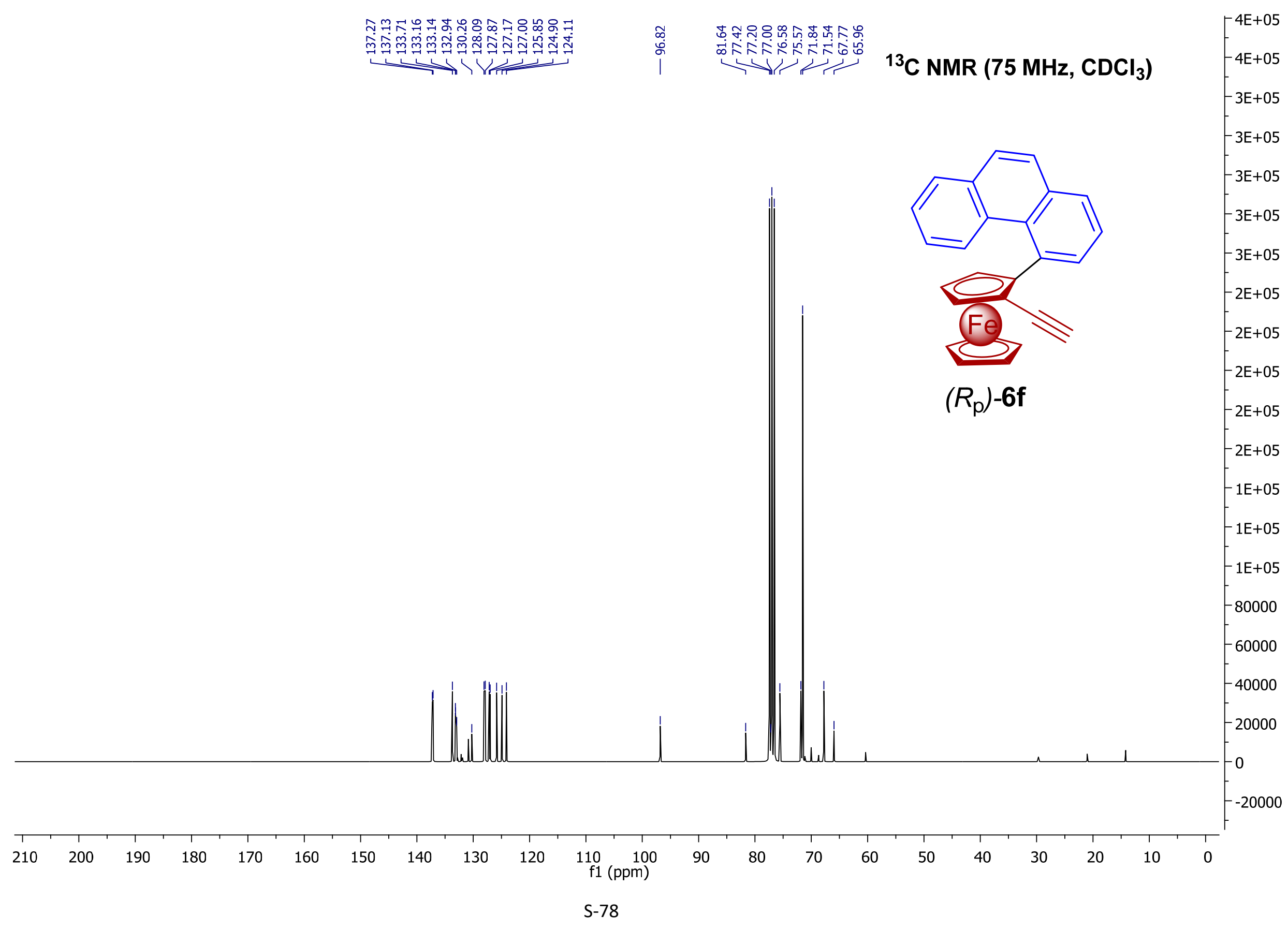




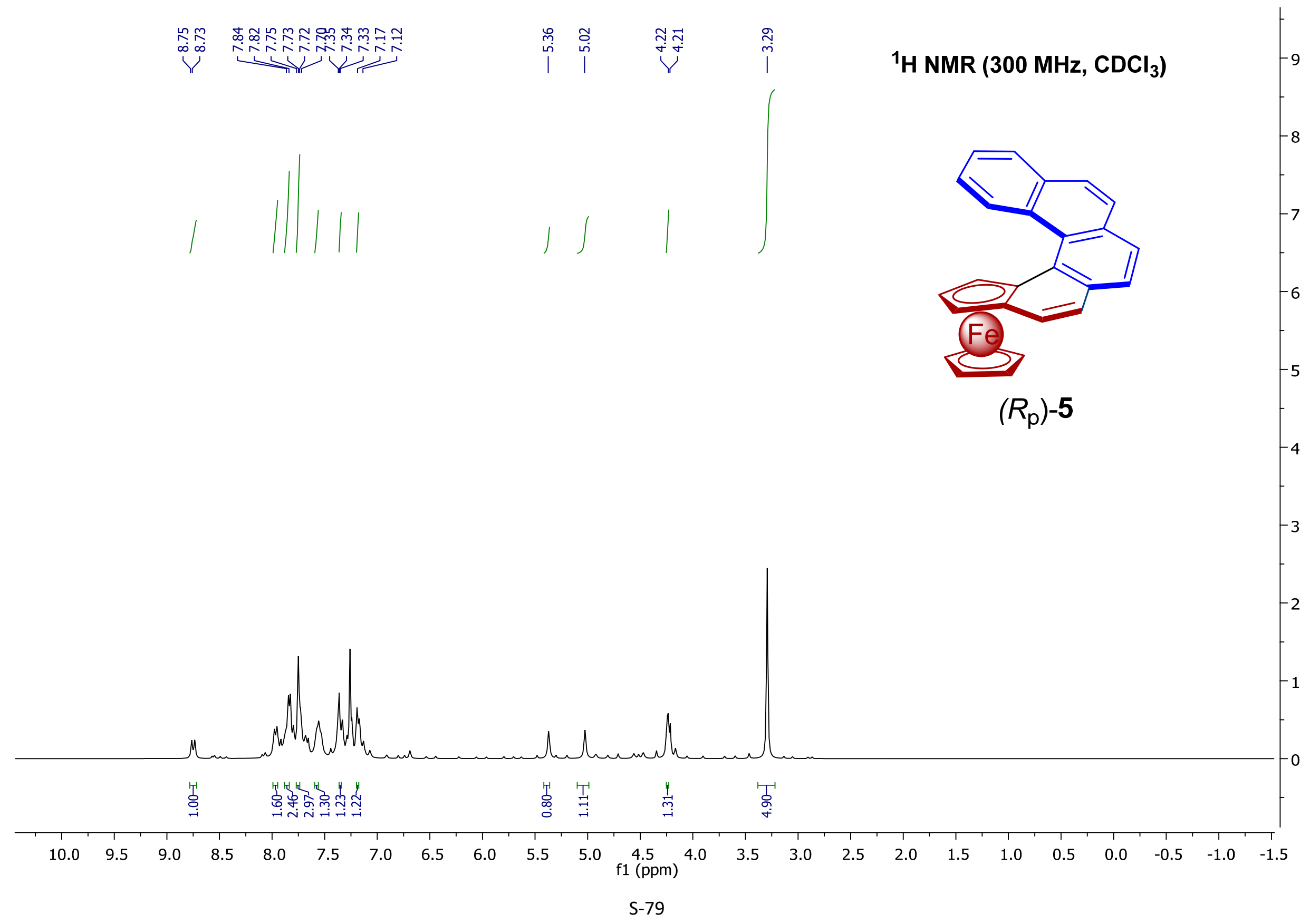




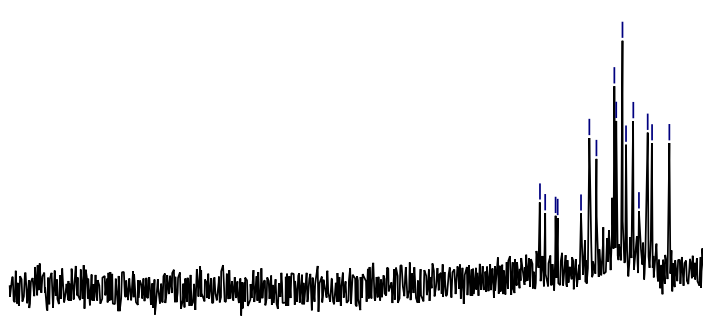

